\title{
MONITORING OF DOWN-HOLE PARAMETERS FOR EARLY KICK DETECTION
}

\author{
by \\ (c) Ayesha Arjumand Nayeem \\ A Thesis submitted to the \\ School of Graduate Studies \\ in partial fulfillment of the requirements for the degree of \\ Master of Engineering \\ Faculty of Engineering and Applied Science \\ Memorial University of Newfoundland
}

May- 2016

St. John's, Newfoundland and Labrador 


\begin{abstract}
The sudden hydrocarbon influx from the formation into the wellbore poses a serious risk to the safety of the well. This sudden influx is termed a kick, which, if not controlled, may lead to a blowout. Therefore, early detection of the kick is crucial to minimize the possibility of a blowout occurrence. There is a high probability of delay in kick detection, apart from other issues when using a kick detection system that is exclusively based on surface monitoring. Down-hole monitoring techniques have a potential to detect a kick at its early stage. Down-hole monitoring could be particularly beneficial when the influx occurs as a result of a lost circulation scenario. In a lost circulation scenario, when the down-hole pressure becomes lower than the formation pore pressure, the formation fluid may starts to enter the wellbore. The lost volume of the drilling fluid is compensated by the formation fluid flowing into the well bore, making it difficult to identify the kick based on pit (mud tank) volume observations at the surface.
\end{abstract}

This experimental study investigates the occurrence of a kick based on relative changes in the mass flow rate, pressure, density, and the conductivity of the fluid in the down-hole. Moreover, the parameters that are most sensitive to formation fluid are identified and a methodology to detect a kick without false alarms is reported.

Pressure transmitter, the Coriolis flow and density meter, and the conductivity sensor are employed to observe the deteriorating well conditions in the down-hole. These observations are used to assess the occurrence of a kick and associated blowout risk. Monitoring of multiple down-hole parameters has a potential to improve the accuracy of interpretation related to kick occurrence, reduces the number of false alarms, and provides a broad picture 
of down-hole conditions. The down-hole monitoring techniques have a potential to reduce the kick detection period.

A down-hole assembly of the laboratory scale drilling rig model and kick injection setup were designed, measuring instruments were acquired, a frame was fabricated, and the experimental set-up was assembled and tested. This set-up has the necessary features to evaluate kick events while implementing down-hole monitoring techniques. Various kick events are simulated on the drilling rig model. During the first set of experiments compressed air (which represents the formation fluid) is injected with constant pressure margin. In the second set of experiments the compressed air is injected with another pressure margin. The experiments are repeated with another pump (flow) rate as well.

This thesis consists of three main parts. The first part gives the general introduction, motivation, outline of the thesis, and a brief description of influx: its causes, various leading and lagging indicators, and description of the several kick detection systems that are in practice in the industry. The second part describes the design and construction of the laboratory scale down-hole assembly of the drilling rig and kick injection setup, which is used to implement the proposed methodology for early kick detection. The third part discusses the experimental work, describes the methodology for early kick detection, and presents experimental results that show how different influx events affect the mass flow rate, pressure, conductivity, and density of the fluid in the down-hole, and the discussion of the results. The last chapter contains summary of the study and future research. 


\section{ACKNOWLEDGEMENTS}

Firstly, I would like to express my sincere gratitude to my supervisors, Dr. Faisal Khan and Dr. Venkatesan Ramachandran for the continuous support, insightful comments, encouragement, and also for the tough questions which propelled me to improve my research skills from various perspectives. Their guidance helped me not only in research but also in writing of this thesis and research papers.

My sincere thanks also goes to Dr. Steve Butt, who provided me an opportunity to join their team and gave access to the Drilling Technology Laboratory and research facilities. I would like to thanks Advanced Drilling Technology Group, particularly Farid Arvani, B. Gillis, for helping me during the design and development of experimental setup, P. Rana and Ming for helping me while conducting the experiments. I would also like to thank Dr. Hinchey, Dr. Aziz, Dr. Salim, and technologist Matt Curtis.

Last but not the least, I would like to thank my family: my parents, my husband, my children, and my siblings for supporting me spiritually throughout my research and my life in general. 


\section{Table of Contents}

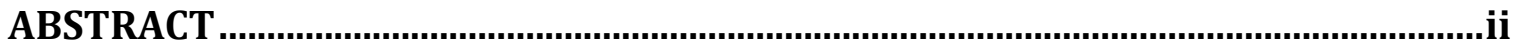

ACKNOWLEDGEMENTS........................................................................................

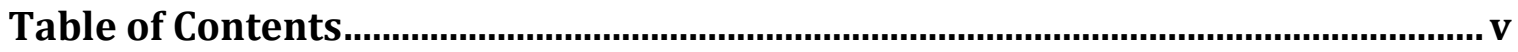

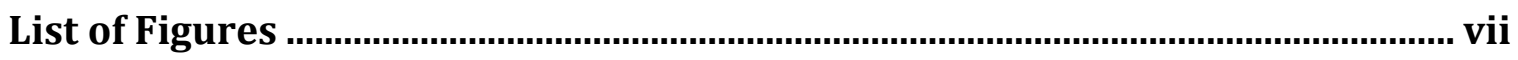

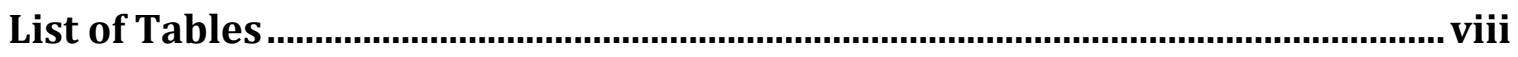

List of Appendices ..................................................................................................... viii

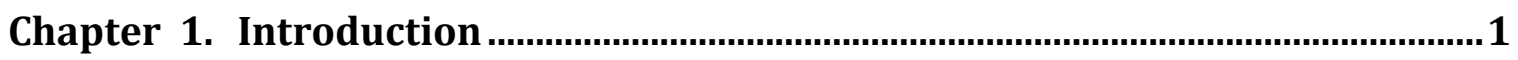

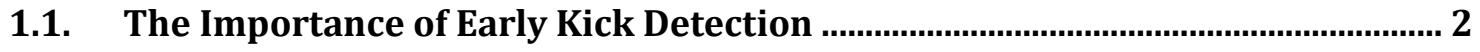

1.2. Limitations of Exclusive Surface Monitoring for Early Kick Detection .............. 3

1.3. The Need for Down-hole Monitoring for Early Kick Detection ............................ 4

1.4. Importance of Down-hole Monitoring in Different Scenarios .............................. 5

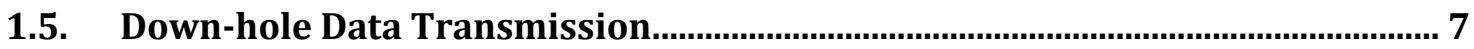

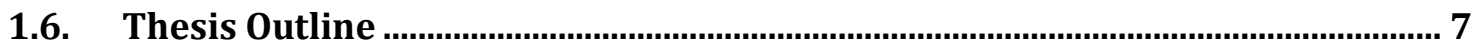

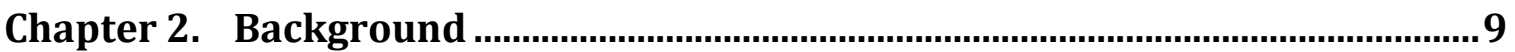

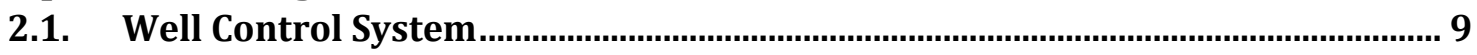

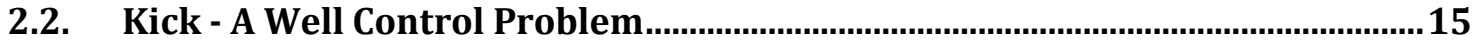

2.2.1. Causes of a Kick ..........................................................................................................16

2.2.2. Kick Indicators .............................................................................................................19

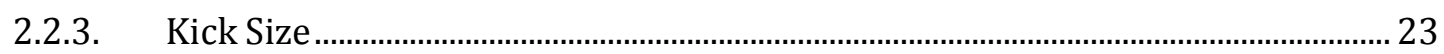

2.3. Description of Kick Detection Techniques ...........................................................24

2.3.1. Mud logging- Conventional Kick Detection Method:_...…………………………2. 25

2.3.2. Kick Detection System Developed by Weatherford.................................................. 30

2.3.3. Kick Detection System Designed by @balance.............................................................33

2.3.4. Flag Fluid Loss and Gain Detection System - Schlumberger ...................................34

2.3.5. Kick Detection System developed by MezurX.........................................................35

2.3.6. Detection of Wellbore Influx and Loss through Pressures .......................................36

2.3.7. Detection of Kick and Loss Incidents using PWD/LWD Tools ................................37

2.3.8. Early Kick Detection System Using Numerical Models:............................................ 39

2.4. Permanent down-hole Monitoring Systems: ........................................................40

2.5. Data Transmission from Down-hole to the Surface ..............................................41

Chapter 3. Design of a Laboratory Scale Down-hole Assembly of the Drilling

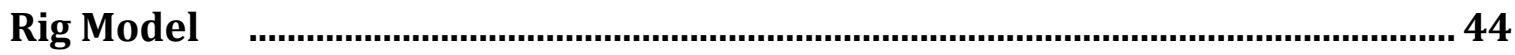

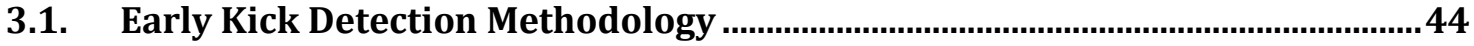

3.2. Structure of Drilling Rig Model.......................................................................47

3.3. Down-hole Sensor Assembly ........................................................................5

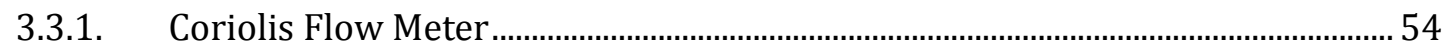

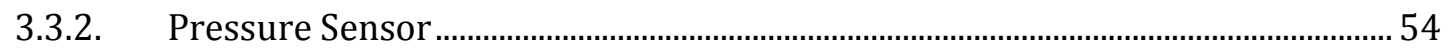

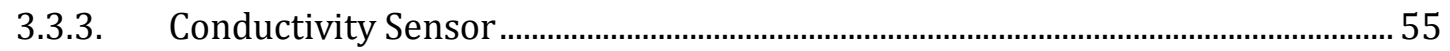




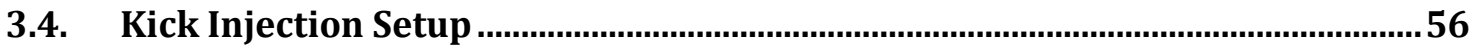

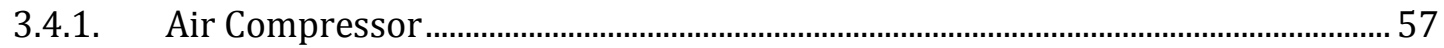

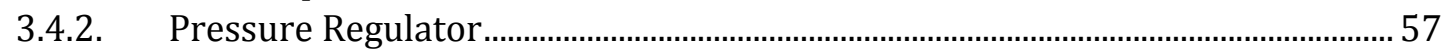

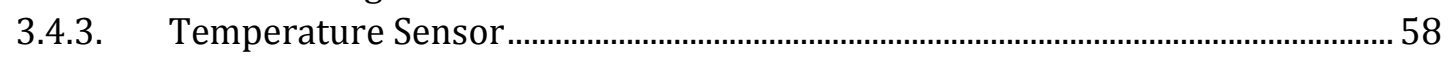

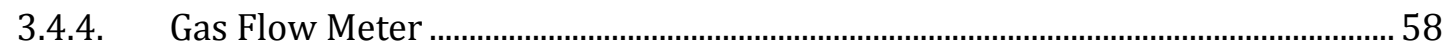

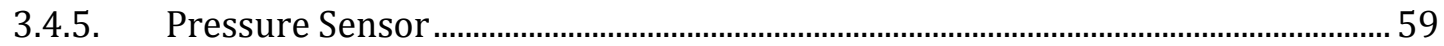

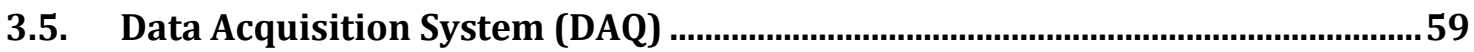

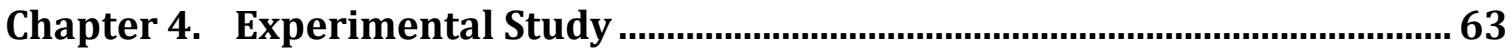

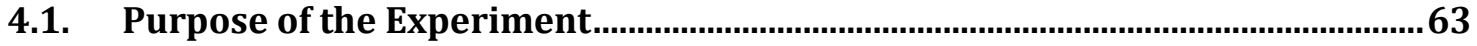

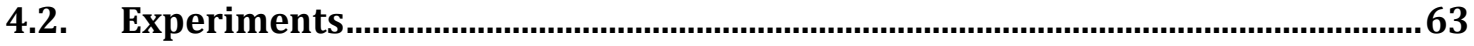

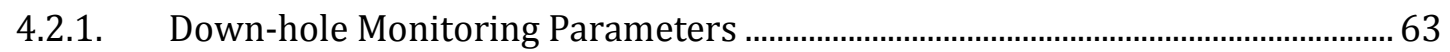

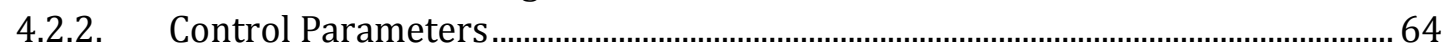

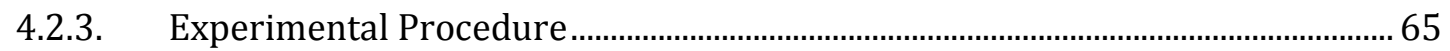

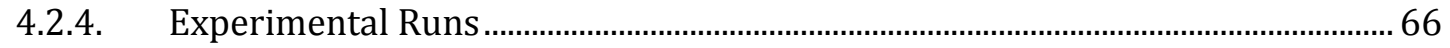

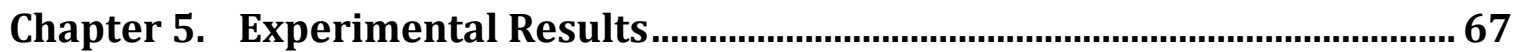

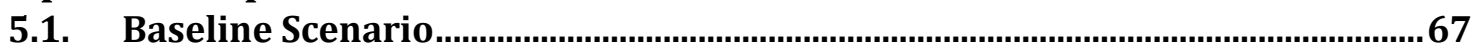

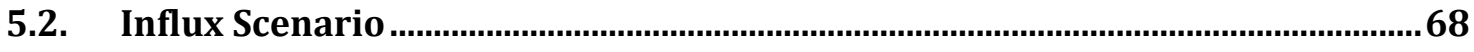

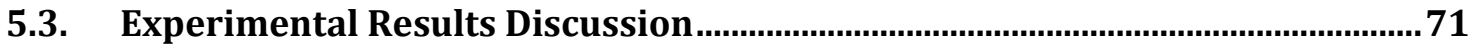

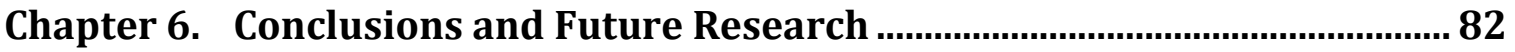

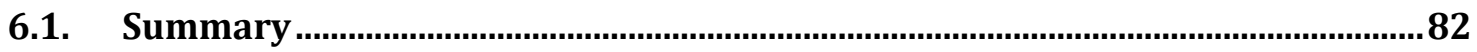

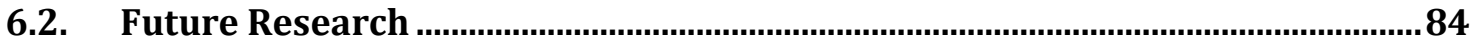

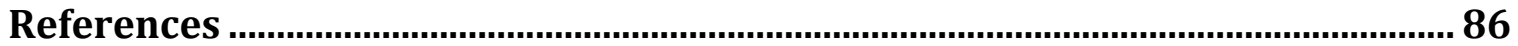




\section{List of Figures}

Figure 1. BOP Stack with Annular Preventer, Pipe Ram, and Blind Ram [1] ................................ 9

Figure 2. BOP Stack with Annular preventers, Pipe Ram, Shear Ram, Drilling Spool, and

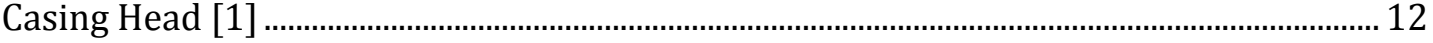

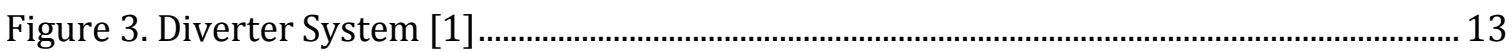

Figure 4. Choke Manifold [1] ........................................................................................................ 14

Figure 5. Microflux Kick Detection System [14] ............................................................................. 31

Figure 6. Microflux Control Manifold [14] .......................................................................................... 32

Figure 7. Piping and Instrumentation Diagram of Experimental Model....................................... 47

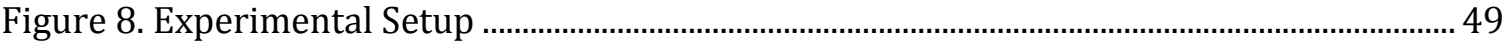

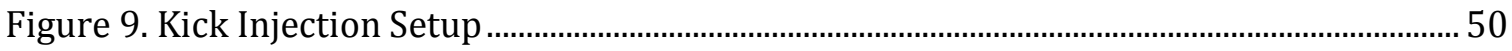

Figure 10. Down-hole Sensor Assembly ………............................................................................... 53

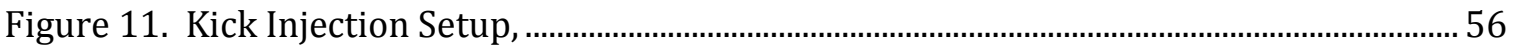

Figure 12. NI 9205 PIN DIAGRAM of 16 Channel Differential Analog Input Module ................ 61

Figure 13. Differential Connection Wiring diagram for one pair of Channels (used for each sensor)

Figure 14. Down-hole Parameter Readings, Baseline Scenario (Steady State Condition) when no Influx occurs

Figure 15. Expected Behaviour of Down-hole Pressure and Mass Flow Rate when Influx Occurs

Figure 16. Expected Behaviour of Down-hole Pressure, Conductivity, and Density when Influx Occurs

Figure 17. Conductivity Variations in the Down-hole During Influx

Figure 18. Mass Flow rate Variation in the Down-hole During Influx 72

Figure 19. Density Variations in the Down-hole During Influx 72

Figure 20. Mass Flow rate after the Influx has stopped 77

Figure 21. The Down-hole Pressure after the Influx has stopped 78 


\section{List of Tables}

Table 1. Differential Channel Pair .......................................................................................................... 61

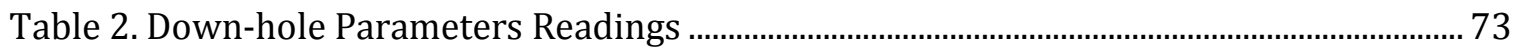

\section{List of Appendices}

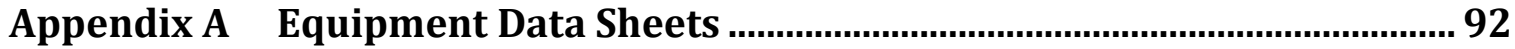

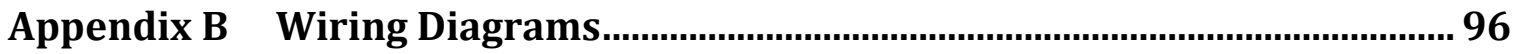

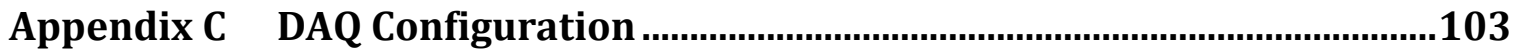

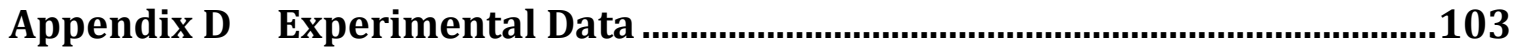




\section{Chapter 1. Introduction}

Oil well drilling is an inherently uncertain process, which is associated with high impact and consequences upon occurrence of an accident. The impacts could be environmental damage, human loss, and monitory loss. Recent experience shows that kick poses the highest risk to the safety of the wellbore. A kick is an undesirable inflow of formation fluids into the wellbore. When kick occurs, the delay in the detection and controlling process may allow the formation fluids to flow uncontrollably leading to a blowout incident. The earlier the detection and control of a kick the better for well safety.

To monitor the down-hole conditions, majority of the early kick detection systems focus on analyzing the returning drilling fluid at the surface. The possibility of blowout prevention could be high if the down-hole monitoring is done along with the surface monitoring. This is because, apart from delay, the exclusive surface monitoring has several limitations, including misrepresentation of the down-hole condition due to: mechanical problems, execution issues, wellbore breathing or ballooning, solubility of the gas in the oil based drilling fluid, and time required to manifest increase in mud tank volume at the surface, thus losing precious reaction time available to the personnel.

The down-hole monitoring technique has a potential to reduce the time taken to identify a kick by monitoring multiple kick indicators in the bottom of the wellbore. The readings from the down-hole sensors can be used to identify deteriorating well conditions and to predict the risk of a blowout. However, the down-hole monitoring is prone to many challenges, including the possibility of excessive false alarms. To limit the frequency of 
false alarm, multiple down-hole parameters could be monitored and interpreted together. This will help to better understand what is actually happening in the down-hole. Monitoring multiple kick indicators in the down-hole might improve the quality of interpretation and can significantly reduce the chances of false alarm.

The focus of this research is to:

- develop a laboratory scale down-hole assembly of the drilling rig model and a kick injection setup.

- investigate the relative pressure changes in the down-hole when influx occurs.

- examine the relative changes in the drilling fluid density and conductivity when influx occurs.

- evaluate the changes in the mass flow rate in the down-hole when influx occurs.

- identify the parameters those are most sensitive to formation fluids.

- develop a methodology to detect the kick without false alarms.

\subsection{The Importance of Early Kick Detection}

During the oil well drilling, even under optimal conditions anything can go wrong at any time. Any accident during drilling operations in the oil and gas industry may result in injury or death to working personnel, along with environmental pollution. Analysis of the well accident data indicates kick as the leading risk to safe well operations. Thus, early kick 
detection is one of the primary safety aspects of oil well drilling operations in the oil and gas industry. A kick is an undesirable inflow of formation fluids into the wellbore. A kick can happen when the hydrostatic pressure in the wellbore becomes less than the pressure encountered within the rock being drilled. Formation fluids enter the wellbore when a barrier, such as mud or cement, fails to resist the high pressure caused by the formation fluids. If formation fluids are not detected and controlled properly in a timely manner, then the inflow of formation fluids becomes uncontrollable, this phenomenon is called blowout. Thus, early detection of fluid influx (kick) from formation is crucial to minimize the possibility of transforming a kick into a blowout incident. Blowouts may cause enormous negative environmental impacts along with very high monetary impact. Early kick detection is crucial in order to prevent huge financial, environmental, and human losses and to reduce non-productive time. Early kick detection also increases the probability of resuming normal operations. In many instances, early detection of fluid influx may give the opportunity to avoid many blowout incidents.

\subsection{Limitations of Exclusive Surface Monitoring for Early Kick Detection}

Kick does not turn into blowout instantaneously. Most often, when influx occurs, it takes some time to evolve into a critical incident such as a blowout. Kick detection systems that exclusively rely on the surface measurements of the kick indicators might delay the kick detection process. The possibility of blowout prevention could be high if the down-hole monitoring is done along with the surface monitoring. This is because most often there is a time lag between kick initiation and the identification of any abnormality at the surface. 
Apart from delay, the exclusive surface monitoring may become inadequate due to many reasons. For example, surface equipment failure, such as improper functioning of flow meters, may allow a kick to remain unidentified. The increase in mud tank volume at the surface also takes some time to manifest. Sometimes execution issues, such as an incorrect lining up of trip tanks, may give incorrect information regarding wellbore conditions in the down-hole. Mechanical problems, such as plugged pipelines and hydrate formation may also interrupt the kick monitoring at the surface. When formation fluid enters the wellbore and if the gas is soluble in the oil based drilling fluid then formation gas remains unnoticed until it breaks out of the drilling fluid. Since most often the separation of gas and drilling fluid occurs at low depths, the gas may reach above the Blow Out Preventer (BOP) before it is identified. Wellbore breathing or ballooning is sometimes confused with the kick. When wellbore is over-pressured (wellbore pressure is greater than the formation pressure), the formation may crack and the drilling fluid is lost in the formation cracks. When the pressure in the wellbore decreases, the lost drilling fluid in the formation cracks flows back into the wellbore. This phenomenon is called wellbore breathing or wellbore ballooning. If the focus is on monitoring kick indicators only at the surface, then this scenario sometimes can be confused with a kick [1].

\subsection{The Need for Down-hole Monitoring for Early Kick Detection}

The need for down-hole monitoring is specifically important when the influx occurs as a result of a lost circulation scenario. In the lost circulation scenario when the down-hole pressure becomes lower than the formation pore pressure, the formation fluid may starts to enter the wellbore. When the lost volume of the drilling fluid is compensated by the 
formation fluid flowing into the wellbore, it becomes difficult to identify the kick based on flow rate or pit volume observations at the surface. Because, the increase in flow rate and pit volume at the surface is only noticed when the loss of drilling fluid is overcompensated by the inflow of formation fluid. However, a significant amount of fluid influx would have already entered the wellbore.

The long choke lines in deep water subsea systems create a considerable frictional effect. Unexpected pressure fluctuations, due to this frictional effect, may allow the gas to break out of the solution. The released gas displaces the drilling fluid inside the choke line, which is then replaced by kill fluid. The resulting pressure variation due to the displacement may cause substantial variation in down-hole pressure [1].

\subsection{Importance of Down-hole Monitoring in Different Scenarios}

First Scenario: When formation fluid starts to enter the wellbore, the density of the fluid in the down-hole decreases. One of the main functions of the drilling fluid is to provide the hydrostatic pressure to prevent formation fluids from entering the wellbore. The decrease in the density of the fluid due to the addition of formation fluid in the down-hole causes the hydrostatic pressure to decrease. Thus, with the decrease in the hydrostatic pressure, the down-hole pressure decreases. If kick is not detected early and proper actions are not taken accordingly then the wellbore becomes severely underbalanced, that allows more formation fluids to enter the wellbore. 
The down-hole monitoring has a potential to reduce the time taken to detect the kick compared with the surface monitoring. Early detection of fluid influx in the bottom of the wellbore may restrict further inflow of formation fluid into the wellbore.

Second Scenario: Lost circulation normally happens when there is an insignificant pressure margin present between formation pore pressure and formation fracture pressure. When overall down-hole pressure increases, it may exceed the formation fracture pressure and drilling fluid might be lost in the formation. If drilling fluid is lost in the formation, hydrostatic pressure decreases and the wellbore pressure may become less than the formation pore pressure, generating an underbalanced condition. When a wellbore becomes underbalanced, influx may initiate at any point of time. When influx begins, formation fluid starts to substitute the amount of drilling fluid lost in the formation. The physical properties of the drilling fluid, such as the density, temperature, and electrical conductivity, would be different from those of formation fluids. Thus, when formation fluid replaces the lost volume of the drilling fluid, the physical properties of the fluid in the down-hole would modify. The formation fluid flowing into the wellbore compensates the lost volume of the drilling fluid. This makes it difficult to identify the kick based on flow rate or pit volume observations at the surface until the lost volume of the drilling fluid is overcompensated by the inflow of formation fluid. However, a substantial amount of formation fluid would have already entered the wellbore.

Therefore, the down-hole measurement can provide quicker information related to changing down-hole conditions when compared to surface parameters. 


\subsection{Down-hole Data Transmission}

Traditional mud pulse telemetry is used to transmit the down-hole measurements data to the surface. However, the traditional telemetry techniques for data transmission is possible only when the pumps are running, and during the period of time when pumps are off they store the data and transmit it later when circulation begins. The electromagnetic telemetry technique allows data transmission without a continuous drilling fluid column. This telemetry technique provides an alternative to mud pulse telemetry technique. The electromagnetic telemetry technique establishes a two way communications link between the surface and the tool down-hole. Mud pulse telemetry and electromagnetic telemetry techniques have limited data transmission rates. Wired or Networked Drill String offers the data transmission at a very high speed and without any interruption, independent of circulation condition. It also give an opportunity to transmit in both directions without any disruption even when the circulation stops. Additionally, this feature gives an opportunity to control the down-hole tools from surface.

\subsection{Thesis Outline}

This thesis is organized into 6 chapters. Chapter one gives general introduction including background information, motivation, and outline of the thesis. Chapter two discusses kick (influx): its causes, various leading and lagging indicators, and description of the several kick detection systems that are in practice in the industry. Chapter three consists of description of the design of laboratory scale down-hole assembly of the drilling rig model and kick injection setup that is used to implement the proposed methodology for early kick 
detection. Chapter four presents the purpose of the study, discusses the methodology for early kick detection, and experimental procedure. The fifth chapter presents experimental results that show how influx affects the mass flow rate, pressure, conductivity, and density of the fluid in the down-hole and also provide discussion of the results. The closing chapter, chapter six contains conclusion and future recommendation. 


\section{Chapter 2. Background}

\subsection{Well Control System}

One of the primary objective of a well control system is to manage and safely control the unexpected flow of formation fluids into the wellbore. It will minimize the dangerous

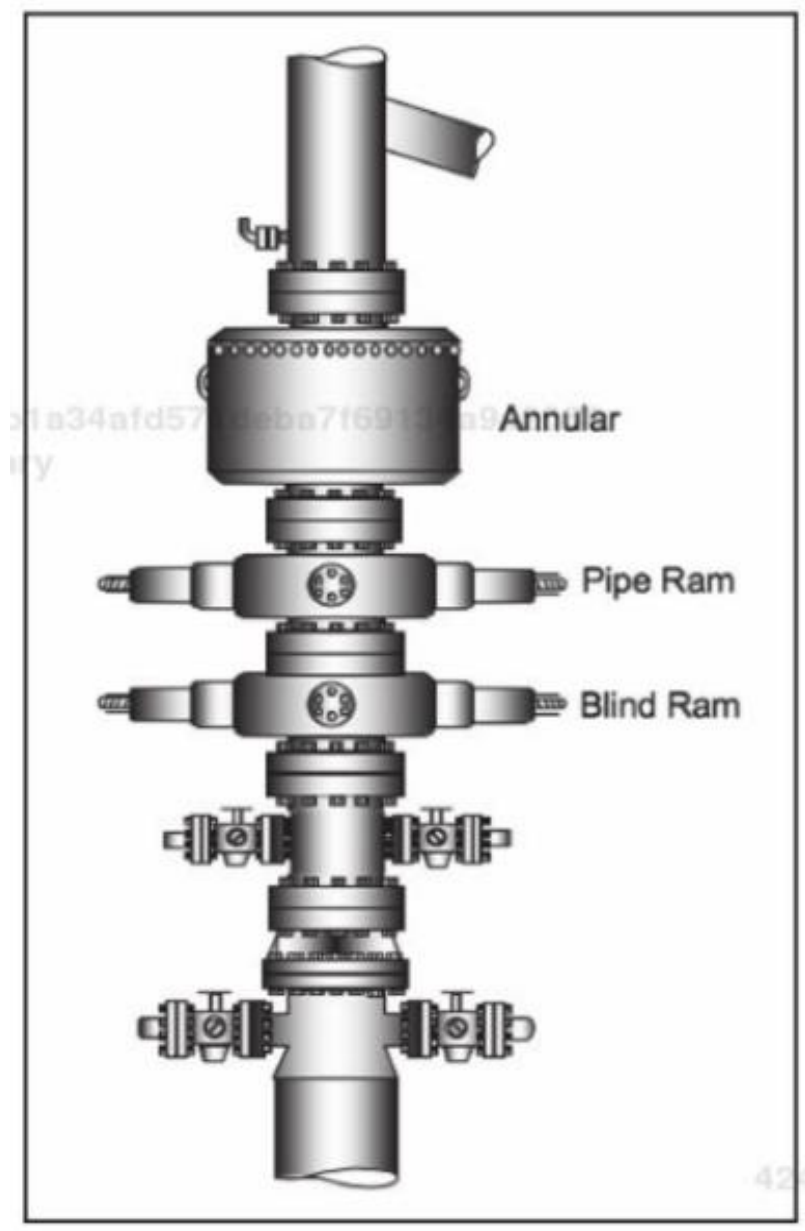

Figure 1. BOP Stack with Annular Preventer, Pipe Ram, and Blind Ram [1] 
effects caused by the influx and eventually prevent the kick evolving into a blowout. Improper handling of formation fluid may allow a small kick to turn into a big blowout.

The well control systems are designed:

$\checkmark$ To shut in the well.

$\checkmark$ To remove the formation fluid.

$\checkmark$ To replace the lighter drilling fluid with heavier drilling fluid.

$\checkmark$ To strip the drill pipe into or out of the hole.

However, well control procedures vary slightly from rig to rig and also on the company policy as well.

Basic components of a well control system are [1]

- BOP stack

- Annular Preventer

- Ram Preventers

- Spools

- Internal preventers

- Casing head

- Kick/choke lines and connections

- Facilities for mud and gas handling (separator)

- Accumulators

- Diverter system 
- Choke manifold

- Buffer tank

- Degaser

- Monitoring Equipment

Annular Preventers: These are made of synthetic rubber that pack the well bore (shown in Figure 1) [1].

Ram Preventers: Ram preventers are of three types (Figure 2) [1]:

- Pipe Ram/casing Ram: It helps to close the annular space around the casing or drill pipe.

- Blind ram: It helps to close the annulus when no pipe is present in the wellbore.

- Shear Ram: During the well control operation shear rams are used to shear the drill pipe when the string is present in the well.

Drilling Spools: These drill-through-type fittings are placed in the BOP stack. It provides a space between the two consecutive pipe rams: to temporary store the tool joints during tripping operations and to attach the kill and choke lines [1].

Casing Head: It helps to seal the casing string and support the BOP stack [1]. 


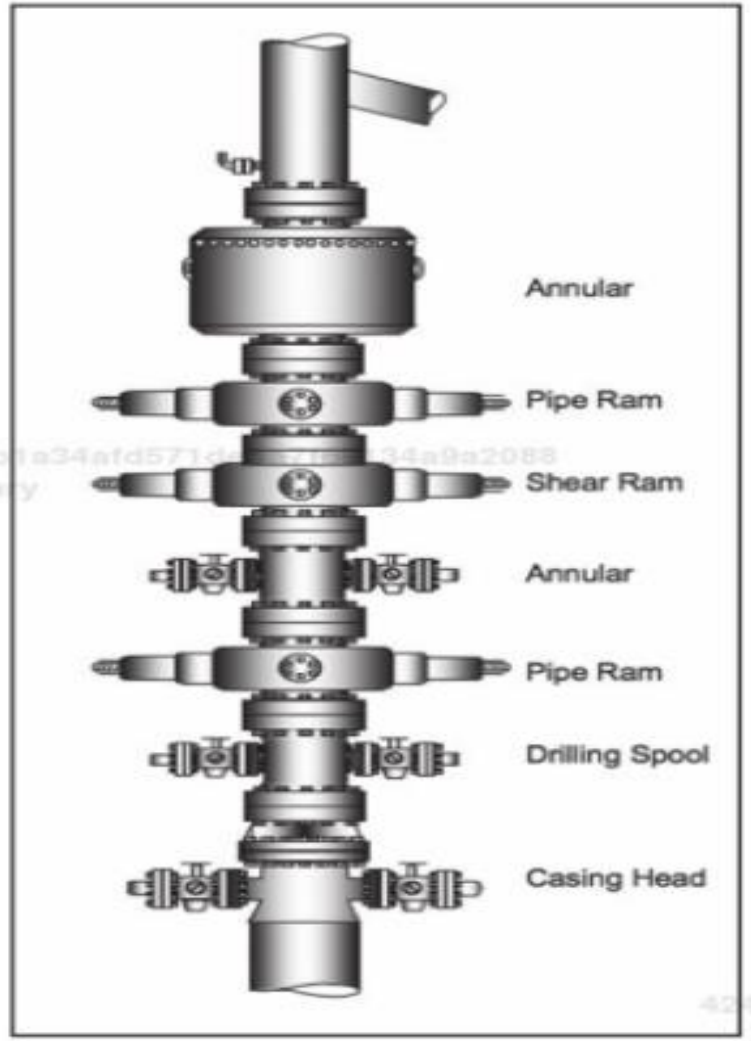

Figure 2. BOP Stack with Annular preventers, Pipe Ram, Shear Ram, Drilling Spool, and Casing Head [1]

Diverter System: This is installed at the top of BOP. To transfer the drilling fluid away from the rig floor, the diverter system diverts the formation fluid to flow through bigger diameter vent lines (shown in Figure 3) [1]. 


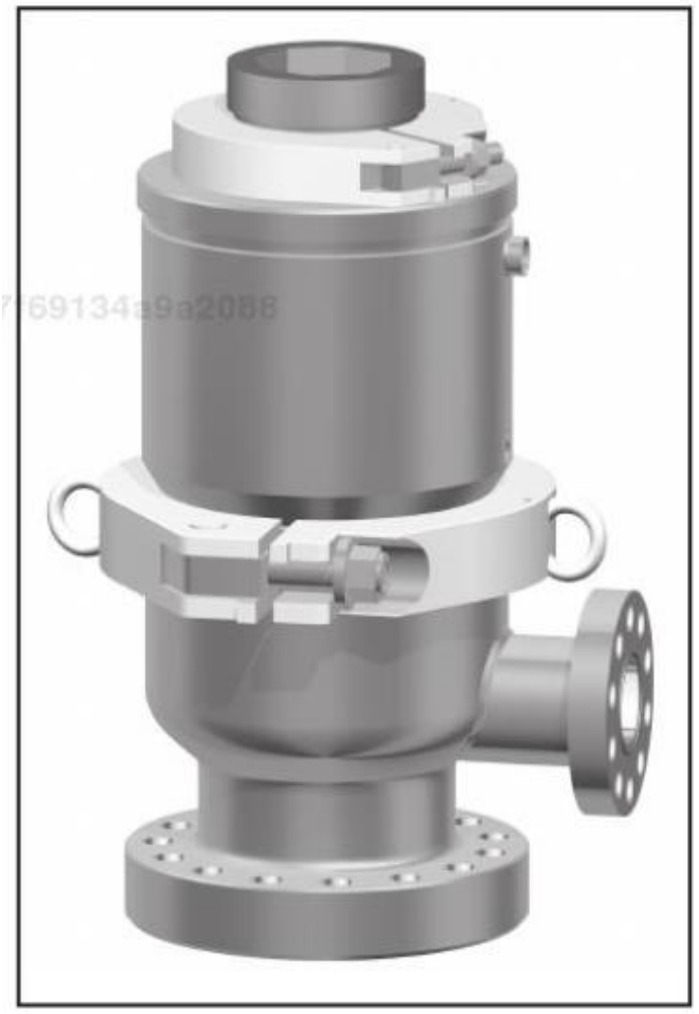

Figure 3. Diverter System [1]

Choke/Kill Lines: The external circulation system used during the well control operation other than the normal circulation system are called as choke/kill lines [1].

Choke Manifold: A choke manifold consists of chokes, valves, and lines. It allows the controlled release of formation fluids from the wellbore (shown in Figure 4) [1]. 


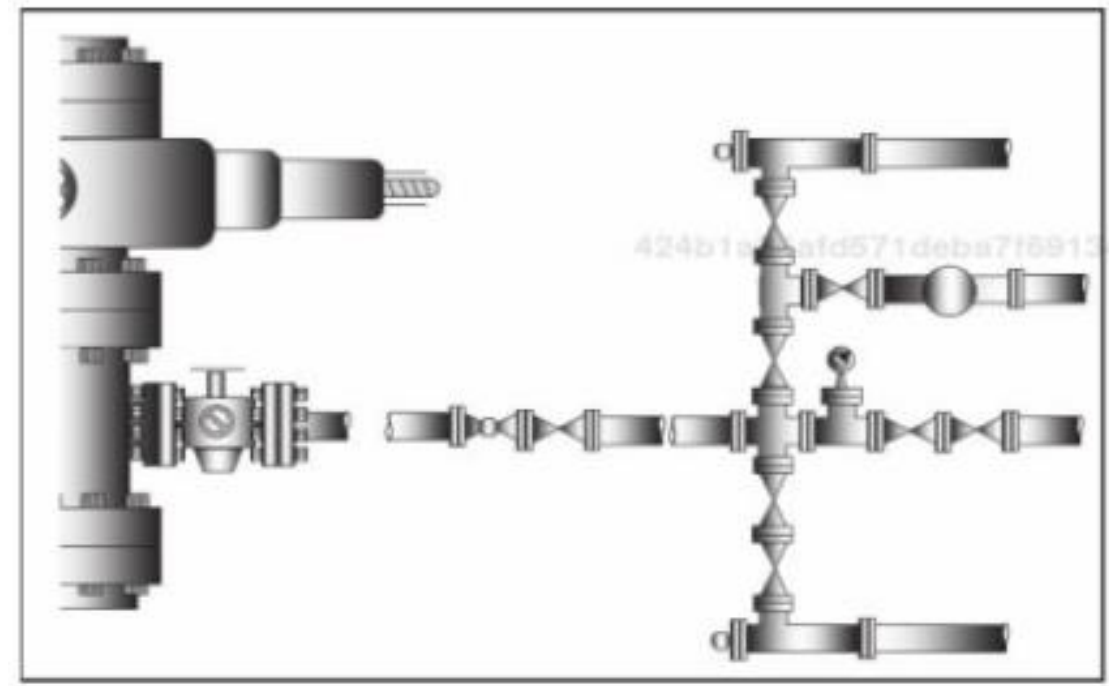

Figure 4. Choke Manifold [1]

Buffer Tanks: In the downstream of choke manifold, the buffer tanks allow the gas expansion.

Degaser: It is used to separate the gas from the drilling fluid [1].

To control the formation fluid inflow the following steps are taken:

The shut-in drill pipe and casing pressure are recorded

The encountered formation pressure is calculated

Required kill fluid weight is calculated

Required circulating drill pipe pressure or scheduled pump pressure during well control is calculated.

Induced wellbore pressure profile, last casing shoe pressure and at the surface choke pressure during well control are calculated. 
Operating pressure limits of well control equipment are set.

$>$ The composition of the formation fluid is determined or guessed.

$>$ The required bottom hole pressure during well control operation are calculated [1].

\subsection{Kick - A Well Control Problem}

In every oil and gas operation problems may occur. This section discusses one of the potential problem kick that may lead to a well control event.

If the pressure exerted by the drilling fluid column is not sufficient enough to overcome the pressure exerted by the formation fluids then the formation fluid can enter the wellbore. This unwanted flow of formation fluid into the wellbore is called kick. In other words kick occurs when total pressure inside the wellbore is less than the formation pore pressure. During underbalanced condition formation fluids start to enter the wellbore if the porous formation being drilled has mobile fluid and there is a permeability to sustain a flow of formation fluids into the wellbore [1]. If the rocks have high permeability and porosity, then the severity of the kick will be higher. Another important factor that affects the severity of the kick is the pressure differential between down-hole pressure and formation pore pressure. If formation pore pressure is very high compared to down-hole pressure, then there will be a very high negative pressure differential. If the negative pressure differential occurs in porous and permeable zones, then a severe influx may occur [1]. Rock strength is an important well control consideration to prevent the formation fluid from entering the wellbore. Rock strength is the maximum pressure that the formation rock can withstand without creating any fractures. Rock strength is often described in equivalent mud weight. 
Equivalent mud weight is the total pressure exerted on the formation wall of the wellbore. It includes hydrostatic pressure, frictional pressure generated due to the drilling fluid pumping, pressure surges created due to the pipe tripping operations, and casing pressure. If total pressure exerted on the wall exceeds the rock strength, then formation may generate a crack that might allow the formation fluid to enter the wellbore.

Some of the known kick fluids are natural gas, hydrogen sulphide gas, salt water, carbon dioxide, oil, and magnesium chloride. Kicks are characterized either based on the volume of the formation fluid or by the amount of extra weight of the drilling fluid required to control the influx to prevent it from evolving into critical incident such as blowout [1].

\subsubsection{Causes of a Kick}

This section discusses various causes of a kick. Understanding the causes of the kick benefits in developing effective kick detection strategies. In many instances, if it is detected early blowout incidents can be avoided. Kick may happen due to one or more of the following reasons:

1. Mud weight less than the formation pore pressure.

2. Failure to keep the hole full while tripping.

3. Swabbing while tripping.

4. Lost circulation.

5. Mud cut by gas, water, or oil.

1. Mud Weight/Hydrostatic Pressure Less than the Formation Pore Pressure: 
In some areas where formation productivity is historically low, drilling is done with a hydrostatic pressure less than the formation pressure to increase the penetration rate. In recent years, the ability to predict formation pressure has improved tremendously, but since geologic correlation mostly relies on interpretation, it is very difficult to predict the formation pressure especially in salt domes. Thus, in some places there is a possibility that unexpected abnormal formation pressure zones may be encountered at any point of time during the drilling process. Since unexpectedly high formation pressure shallow gas sands plague many areas, sudden encounters with abnormal formation pressure zones may allow formation fluids to enter the wellbore [2].

2. Failure to Keep the Hole full while Tripping:

When the pipe is being taken out of the wellbore then the absence of pipe will displace the mud, which then causes a reduction in the mud level in the wellbore. So, during the tripping operation, if the hole is not filled properly then it may allow an inflow of formation fluid into the wellbore. With the help of pump stroke measurements and trip tank, hole fill ups are monitored [1].

\section{Swabbing While Tripping and Surging:}

When the drill bit and bottom hole assembly are pulled out of the hole a piston effect is created that results in a pressure drop before the displaced steel volume is substituted with drilling mud. This effect is called swabbing. Other than the pressures in the formation and the wellbore, there are two main factors that affect the swabbing: the difference between the inside diameter of the open hole and the outside diameter of the pipe being moved, and 
the speed at which the pipe is being pulled. The risk of swabbing increases when there is a smaller difference present between the hole size and the pipe size. Likewise, when the pipe is pulled out with high speed a larger piston effect is created, which may allow the reservoir fluid to flow into the wellbore $[1,2]$. Similarly, tripping in the drill string creates a pressure called surge pressure, which causes an increase in wellbore pressure. Low swab pressure can lead to a kick and high surge pressure can lead to a lost circulation [1].

\section{Lost Circulation:}

When drilling in an overbalanced condition sometimes drilling fluid is being lost in the formation. This results in reduction of hydrostatic pressure. The decreased hydrostatic pressure may allow any permeable formation, which holds fluids with greater pressures, to enter the wellbore. When this happens the lost volume of the drilling fluid is compensated by the formation fluid flowing into the well bore making it difficult to identify the kick based on pit volume observations at the surface. The increased flow rate or pit volume at the surface is only noticed when the loss of drilling fluid is overcompensated by the inflow of formation fluid. However, by that time a significant amount of fluid influx would have already occurred in the wellbore. This may lead to a very difficult well control scenario [1, $2]$.

\section{Mud Cut by Gas, Water or Oil:}

While drilling into productive oil or gas zones, the formation gas may mix with the drilling fluid. Whenever this happens it is treated as a warning signal, however, it might not always cause a serious problem. Due to the compressibility of the gas, severely gas cut mud also 
decreases the down-hole pressures. When an incompressible fluid, such as oil or water, is mixed with the drilling fluid the reduction in the hydrostatic pressure will be more severe and may lead to very serious well control problems [2].

\subsubsection{Kick Indicators}

The wellbore kick indicators can be broadly classified as leading indicators and lagging indicators. Leading indicators of a kick are the early signs of potential occurrence of a kick. Lagging indicators of a kick are the physical and chemical changes in the wellbore that follow an influx in the well bore system. Leading indicators of a kick, such as abnormal variations in density, pressure, and temperature, give advance warning of influx and its potential outcome. The early warning helps in preventing the influx. Lagging indicators give the information about the influx and give the details how much or how bad it has affected the process environment. In other words lagging indicators provide indication of the various effects, which follow an influx. It will not help in preventing the influx, but could perhaps be useful to prevent a blowout.

Leading Indicators of a kick: Some of the main leading indicators of a kick include improper drilling fluid density either low or high, insufficient drilling fluid level, very low swab pressure, very high surge pressure, lost circulation, and indicators of unexpected abnormal high pressure zones. All safety parameters need to be monitored collectively because individual parameters may not always accurately provide the warning signs of a potential kick. Some of the leading indicators of a kick are discussed in the subsequent sections: 
a. Improper Drilling Fluid Density: Less dense drilling fluid causes hydrostatic pressure to decrease to values that may be inadequate to prevent the formation fluids from entering the wellbore. Several issues can cause the drilling fluid density to be improper to prevent the influx such as accidental dilution or excessive dilution of drilling fluid, gas in the mud, barite settling in the down-hole, heavy rains in the pits, sweep, and low density pills in the well [1].

b. Abnormal Pore Pressure Zones: There is always some uncertainty present in predicting formation pore pressure. So, even while drilling into known normal pressure zones, sometimes abnormal high pore pressure zones are encountered. If the increased formation pore pressure is greater than the down-hole pressure, then there is a high possibility that influx can start at any time. Warning signs of abnormal high pore pressure is a leading indicator of a kick [2]. Some of the warning signs of abnormal pore pressure are:

1. Decrease in Shale Density: Generally, the density of formation shale increases with depth because of increased compaction reduces fluid volume and porosity. But as soon as an abnormal high-pressure zone is encountered, the density of shale formation starts to decrease. This happens because an increase in pore pressure within the shale prevents compaction [3].

2. Increase in Cutting Size and Shape: Underbalanced situations developed by abnormal high pore pressure zones can cause the formation to break. Since broken cuttings are not ruined by a drill bit they are more sharp and big in comparison to normal cutting size [3]. 
3. Decrease in d-exponent Value: Generally, as drilling progresses, the d-exponent trends will increase, but a decrease in this trend may be an indication of abnormal high pore pressure zones [3]. The d-exponent equation was developed to normalize the penetration rate. The d-exponent is calculated using the following equation [1]:

$\mathrm{d}=\left(\log \left(\frac{R}{60 \times N}\right) / \log \left(\frac{12 \times W}{1000 \times D}\right)\right) \times\left(\mathrm{P}_{n} / \mathrm{P}_{\mathrm{m}}\right)^{\mathrm{c}}$

R: Penetration Rate

N=Rotary Speed

$\mathrm{W}=$ Weight on Bit

$\mathrm{D}=$ Drill Bit Diameter

$\mathrm{P}_{\mathrm{n}}=$ Mud Weight Equivalent

$\mathrm{P}_{\mathrm{m}=\text { Mud Weight used }}$

$\mathrm{C}=$ Shale Compatibility Coefficient

4. Increase in Return Mud Temperature: A temperature increase above the normal trend can be an indicator of abnormal high pore pressure zones. There are certain parameters which need to be considered while interpreting the temperature changes such as, circulation rate, wellbore geometry, chemicals or solid content in the mud, and increased bit torque $[2,3]$.

5. Increase in the Drag and Torque: Drilling into over-pressured formation increases drilling torque and drag $[2,3]$.

6. Rapid Increase in the Drilling Breaks: The drilling rate mainly depends on the differential between formation pore pressure and down-hole pressure generated by drilling 
fluid (mud). Abnormal increase in the formation pore pressure increases the drilling rate. The sudden increase in the drilling rate may be a leading indicator of a kick [2, 3].

c. Severe Lost Circulation: When the lost circulation happens, hydrostatic pressure gets reduced in the bottom of the well bore. If bottom-hole pressure becomes less than the formation pore pressure, formation fluids may start to flow into the wellbore [2].

d. Insufficient Drilling Fluid Level: During the tripping operation of the drill string, improper drilling fluid level in the wellbore can reduce the down-hole pressure. If it is less than the formation pore pressure, formation fluid can enter the wellbore [2].

Lagging Indicators of a Kick: The primary lagging indicators of a kick include:

a. Increase in Mud Tank Volume: When influx begins formation fluids replaces the drilling fluid in the wellbore. This will increase the mud tank volume. However, it takes some time to manifest the increase in mud tank level at the surface, so it does not immediately give the indication of a kick [1].

b. Increase in Flow Rate: When drilling fluid is pumping at a constant rate and if formation fluid enters the wellbore, it pushes the drilling fluid to move up the annulus. So the resulting increase in the flow rate is the key indicator of a kick [2].

c. Flowing Well: When the pump is not pumping the mud, continued flow of the drilling fluid out of the wellbore is another primary indicator of a kick/influx [1].

The secondary lagging indicators of a kick include: 
a. Increase in Drill String Weight: Drilling fluid creates a buoyant effect on the drill string. This results in the decrease of the actual pipe weight. When less dense formation fluid enters the wellbore it decreases the buoyancy. Due to the low buoyant force on the drill string the weight of the drill string increases. However, the change in the drill string weight might be small, so it needs to be monitored carefully to detect the kick.

b. Changes in Pump Pressure: A change in pump pressure may give indication of a kick. At the beginning when formation fluid enters the wellbore it might flocculate causing temporary increase in the pump pressure. When the flow of formation into the wellbore continues and low-density formation displaces the heavier drilling fluid, then pump pressure may start to decrease. Due to the less dense fluid in the annulus the drilling fluid in the drill string tends to drop causing increase in the pumping speed. Though other problems also show the same sign it is always important to check for a kick when this condition is observed [2].

c. Changes in Return Mud Properties: Without any addition of other material into the drilling fluid, changes in its properties can be due to mixing of water and/or chloride from formation fluids. Contamination of formation water with oil-based mud will result in a viscosity increase, whereas contamination of formation water with low $\mathrm{pH}$ waterbased mud can decrease the mud viscosity [1].

\subsubsection{Kick Size}

The kick size is determined by the increase in fluid volume at the surface. The volume of the incompressible and insoluble formation fluid at the surface will be equal to the actual kick volume in the down-hole. 
The volume of the kick at the surface depends on two parameters, increased volume at the surface and the down-hole pore pressure. The compressibility of the formation fluid plays an important role, particularly when the formation fluid is a gas. This relationship can be described in terms of ideal gas law: $P_{1} V_{1}=P_{2} V_{2}$, where $P_{1}$ is the pressure and $V_{1}$ is the volume in the down-hole, while $\mathrm{P}_{2}$ and $\mathrm{V}_{2}$ are the pressure and volume at the surface respectively. For example, when a $2 \mathrm{~m}^{3}$ kick is taken with a down-hole pressure of 100 bars it will correspond to $100 \mathrm{~m}^{3}$ at a surface pressure of 2 bar. Due to the expansion of the gas handling of this large amount of gas is a challenge. The capacity of the gas handling system should be good enough to circulate out the kick. If the gas kick is not allowed to expand in the down-hole, the down-hole pressure may contribute to fracture the formation in weak zones of the wellbore [4]. So early detection of fluid influx from formation is very crucial.

As hydrocarbon gas is soluble in the oil-based mud, it exhibits a complex behavior. Due to the solubility of formation gas in oil based mud, a large volume of formation fluid enters the wellbore without any significant increase in the volume at the surface when compared to water-based drilling mud [4]. Apart from the hazard caused due to gas expansion itself, sometimes it might contribute to improper hole filling that may increase the possibility of another formation influx [4].

\subsection{Description of Kick Detection Techniques}

In order to safely control the inflow of formation fluids, the influx must be detected as early as possible. If the kick detection process is delayed, then formation fluids will continue to 
enter the wellbore. This will eventually accelerate into uncontrolled flow of formation fluids leading to a blowout scenario [1].

\subsubsection{Mud logging- Conventional Kick Detection Method:}

Mud logging is the conventional method to monitor and detect the formation influxes. Mud logging evaluates various parameters that give the information about the down-hole condition. Some of the parameters that the mud logger monitors include gas, mud pit volumes, flow back, and pump pressure [2].

Gas: A gas detection system that monitors the drilling mud returning from the well provides valuable information when the down-hole conditions changes. For example, if an increase in the amounts of gas in the drilling fluid is observed after connection has been made then it indicates a decreasing overbalance in the well, and it denotes possible reservoir influx. A gas system usually measures the total amount of gas and also measures (calculates) the different components in the gas. It gives valuable insight into the properties of the downhole fluid. Since the gas is monitored and detected at the surface it needs to be circulated from the bottom of the well to the surface. If the measurements are not taken quickly, it does not give the early indication of kick [2].

Mud Pit Volumes: During drilling operations mud pits are directly connected to the well. A pump draws the drilling mud from one of the mud pits and return flow from the well is collected in the other mud pit. Ideally, after correcting for the amount of hole drilled, the volume of mud in the pits should be maintained constant. If an unexpected increase in the mud pits volume is observed, then it may be because of the influx from the formation. If an 
unexpected decrease in mud volume is observed then it may be because of the loss of drilling mud in the formation. There are, however, certain limitations to mud volume monitoring for kick detection. The reason for this uncertainty is that, even with a good mud treatment system, some quantity of the mud may be lost with the cuttings passing through the shakers. The decrease in the volume of the drilling mud in the pits appears as loss of circulation. The sensor readings may be altered by movement of the floating rigs and also due to the movement of the on board cranes. Another factor that affects the accuracy of the mud pit volume readings is the size of the pit being used. In order to maintain the proper circulation pit volume should be larger, but it may increase the uncertainty [2].

Sometimes kicks are taken during tripping operations. Thus to minimize the uncertainty and to detect the volume changes precisely and quickly, a smaller trip tank is used during tripping operation.

Connection Flow backs: During circulation a certain amount of mud occupies the surface circulation system. This mud will flow back into the pits when the pumps are shut off to make a connection. It may appear as the increase in the mud volume level though no formation fluid is coming inside the well bore. Hence a careful monitoring of the flow rate and the amount of flow back is done to detect any changes in the down-hole condition. Flow back volume should be more or less the same at each connection, and any changes may indicate a change in the down-hole condition [2].

Pump Pressure: The pump pressure gives the information about wellbore pressure and the frictional pressure drop through the well. Pump pressure peaks may suggest down-hole 
problems or lost circulation due to the fracturing of the formation. When a decrease in pump pressure is noticed, then it may give an indication that the fluid, which is flowing through the annulus, becomes light [2].

Drill Breaks: Sometimes a sudden increase in the Rate of Penetration (ROP) happens when the pore pressure is increased. The pressure differential between the wellbore and formation is directly related to the ROP. This pressure differential creates a "hold down" effect, making the formation harder to drill. An increase in pore pressure will reduce this effect, making chips cut by the drilling bit easier, resulting in a higher rate of penetration. Sometimes a change in ROP may be an indication of a change in formation properties, without a change in the pore pressure. Also in some cases a change in ROP may be an indication of a change in both formation properties and pore pressure. An increase in the hook load at the surface is also noticed due to the same reason, i.e., when the hold down effect decreases resulting an increase in ROP.

Return Flow: Formation fluid influx and lost circulation can be noticed through return drilling fluid monitoring. During normal drilling operations the flow in and out of the well will be maintained constant. A change between the flow-in and flow-out indicates unstable conditions. If flow out is less than the flow in, then it is indicated that drilling fluid is lost in the formation. If flow-out is more than the flow-in, then it indicates that formation fluids are entering the well bore [2].

Use of a low accuracy flow meter or a flow meter, which is prone to plugging by the formation cuttings results in the flow out not being a reliable parameter for monitoring. A 
change in the trend is monitored because sometimes even a "good" flow meter may not provide the correct numerical value.

Monitoring Tripping Operation: During a tripping operation, when the drill string is pulled out of the well, the volume of the steel pipe that is removed has to be replaced by mud to prevent the influx. Similarly, while tripping in the drill string, a certain amount of drilling mud is expected to flow back from the well as the drill string is displacing it. During the tripping operations a trip sheet is maintained and the actual volume changes are observed and compared with the calculated values from the pipe data to detect any changes in the down-hole. If volume measured is more than the calculated value, then it is an indication of formation influx and if it is less than the calculated value, then it indicates that the drilling fluid is lost in the formation. These calculations are done automatically [2].

During a tripping operation the well is connected to the trip tank system rather than the mud pits to monitor even a very small volume change involved during the tripping operation. Since monitoring trip tanks of volume $5-10 \mathrm{~m}^{3}$ gives a higher accuracy than monitoring mud pits with an active volume of $50-100 \mathrm{~m}^{3}$ [2].

Flow Meters: An ultrasonic sensor for early kick detection was proposed in [5]. A rolling float outflow meter for kick detection was presented in [6]

Coriolis flow meters have been used in surface kick detection systems for around 20 years [7]. Its accuracy and compatibility with oil and water based fluids makes it usable at various oil well. [8]. When space limitations do not allow installation of a Coriolis flow meter and if a water-based mud is in use an electromagnetic flow meter is used [15]. 
Delta Flow: Delta flow is calculated by subtracting the amount of fluid flow into the well from the fluid that flow out of the well. A positive value indicates that a kick is happening and a negative value indicates that fluid loss has occurred [10]. In steady state theoretical conditions, this appears to be a fairly simple solution to detect kicks, but the drilling process is not a steady state process. Non-steady state transient effects make the normalization of flow measurements a challenge. To compensate for the transient effects in flow measurement, probabilistic detection methods for deep water were proposed in [11]. In order to limit false alarms and increase the detection frequency, the requirement for signal processing and event probability computations was suggested in [12]. Artificial Intelligence with an advanced dynamic flow model was used in the kick detection system [13]. To precisely monitor the down-hole condition in a non-steady drilling process, a complex delta flow computation is required rather than simply subtracting flow in from flow out of the wellbore.

Interpretation of Parameters: Formation influx can be detected by monitoring various parameters. However, in many instances individual parameters do not provide the definitive information. So to get insight of the down-hole condition, the various indicators are interpreted together in order to accurately understand the situation in the wellbore. The driller and mud logger interpret the various parameters together. Manual interpretation is always prone to errors because it depends on the driller and mud logger's training, experience, and knowledge about the specific drilling rig and their interpretation abilities. Thus, automated kick detection is more reliable than human interpretation if the system is designed efficiently. 
The following are some of the existing methods for early detection of a kick that are in practice in the industry. Most of the commercial early detection systems are automatic.

Automated Monitoring of Traditional Parameters during Conventional Drilling: Some of the major kick detection systems designed by Weatherford, @balance, Schlumberger, and MezurX that are in practice in the industry are described in this section. Furthermore some other kick detection techniques including detection of wellbore influx and loss through pressures using pressure while drilling (PWD)/logging while drilling tools (LWD) are described in brief in the following subsections.

\subsubsection{Kick Detection System Developed by Weatherford}

Weatherford offers three types of early kick detection systems, Microflux ${ }^{\mathrm{TM}}$ detection, surface logging, and geo-pressure consulting [14].

Microflux Detection: This advance detection system has three primary components, a rotating control device, a mass flow meter, and an intelligent control unit as shown in Figure 5. All three components with software are assembled in one single piece of equipment compatible with virtually any type of rig. This system can detect the kick and control it as well [14]. 


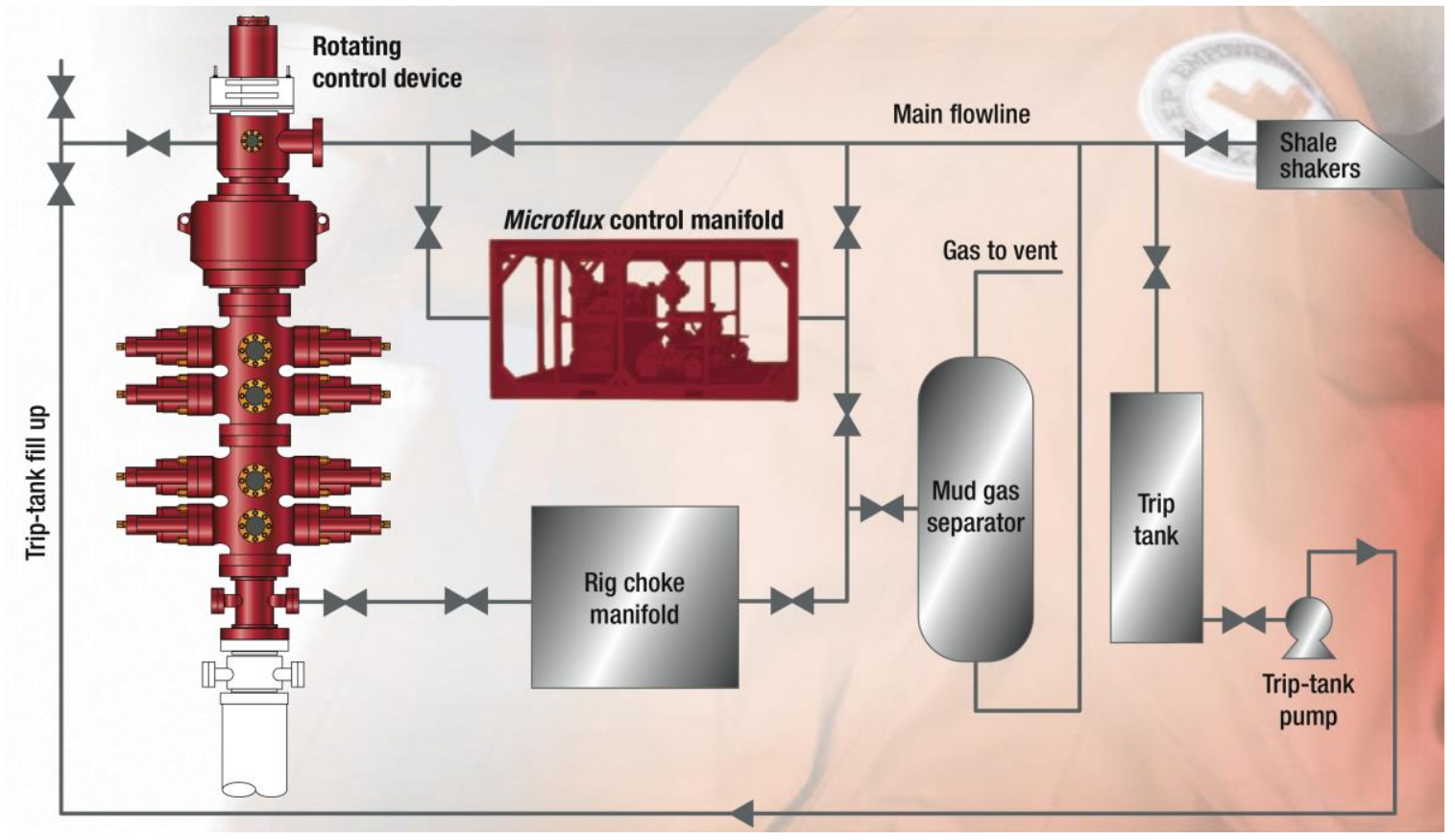

Figure 5. Microflux Kick Detection System [14]

$\mathrm{RCD}$ is used in return systems to divert the annular fluids while drilling. As a part of the Microflux system, it also does a secondary job, i.e., it diverts the fluid through the mass flow meter that measures the various parameters of the fluid.

The Coriolis mass flow meter is used for the real time measurement of the returning annular fluid, mass and volume flow, mud weight, temperature, and density (shown in Figure 6). The data is transmitted to the intelligent control unit. 


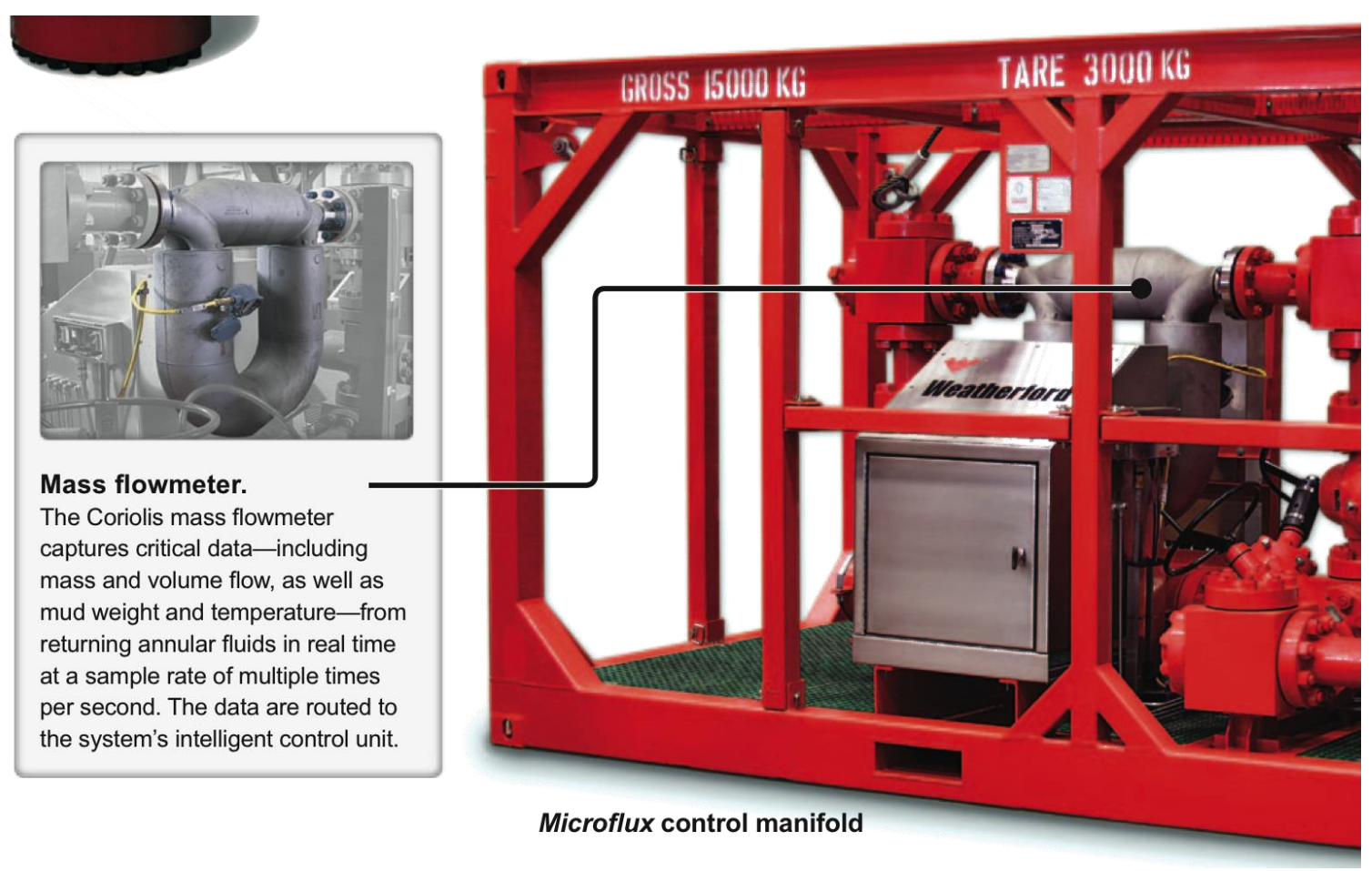

Figure 6. Microflux Control Manifold [14]

The Intelligent Control Unit of Microflux system uses propriety algorithms to identify the minute kick or losses. It can distinguish between influx and ballooning. It also calculates the severity of the influx and other harmful events such as, choke and nozzle plugging, pump efficiency, and surface leaks. The data is displayed on the driller's screen and also on offshore locations.

Surface Logging: Surface logging tools include the flow meter, advanced gas detector, and software. It measures the fluid flow, density of the return mud, and the gas content. The software used in this system is compatible with a variety of flow meters. 
Pit Volume Totalizer enables early kick detection even when the pumps are off. Various kick detection tools are not able to detect the kick when the pit volume increases as a result of flow back (continuous flow out of drilling fluid when pumps are off). The flow back monitor and software in PVT helps to detect the aberration that indicates the kick in many drilling related situations.

GC-Tracer-Surface gas detector is a gas detector at the surface that analyses the formation gas samples regardless of mud type, flow rate, gas solubility, and temperature in the flow line. It is also used as a means of verification of kick detected by other equipment. It will determine the composition of the formation gases so that proper kick killing techniques are used accordingly. It checks the gas levels after the kick is killed to make sure that it is safe to start the normal operation.

Geo Pressure Consulting: The geo pressure consultant uses various sources of information to characterize the formation pressure profile before the drilling operation begins and during the drilling as well. The Predrill Pore Pressure model is used to assess the potential for kick or loss before even starting the operation. During the drilling operation, specific models are used to reassess the risk of an influx by using the real time data acquired through Logging While Drilling (LWD) and Measurement While Drilling (MWD) tools.

\subsubsection{Kick Detection System Designed by @balance}

Kick detection system of @ balance calculates the delta flow using the Coriolis flow meter. The Coriolis flow meter is installed in the return mud flow line downstream of the choke manifold. The kick detection system is equipped with the Coriolis meter. The software 
monitors, plots, and detects the changes in drilling fluid flow in and flow out of the wellbore. Delta flow is continuously monitored and plotted using Human Machine Interface (HMI) to identify the levels at which the system notifies the crew with an alarm. The data is recorded in real time and transmitted using Wellsite Information Transmission Specification (WITS). Stroke rate counters provide the stroke data to calculate the flow in of the drilling fluid. An alarm is issued when the delta flow reaches a certain preselected value [9].

\subsubsection{Flag Fluid Loss and Gain Detection System - Schlumberger}

The Flag system also monitors the delta flow under various operating conditions. Differential flow out is monitored through Coriolis flow meter. A Coriolis flow meter installed in the return mud flow line continuously monitors the actual flow rate. The measured actual flow values are compared with the theoretical values. This will give a quick indication of an influx. The algorithm in the system is used to monitor the influx during drilling, circulation, pipe connection, tripping, and cementing operations. The system automatically compensates for mud compressibility and disturbances that could alter the true flow rate. Along with the visual and audible alarms, computed and measured data are continuously displayed in the log format [15].

Monitoring the flow out rate at the flow line rather than the mud tank provides quick information about the down-hole condition. It can significantly give warning of any abnormality very quickly that allows more time to do control actions. During drilling 
operations the accurate measurement reduces the number of false alarms, thus reducing the amount of non-productive time.

\subsubsection{Kick Detection System developed by MezurX}

The Fluid Anomaly Detection System (FADS): The flow sensors are used for measuring Flow In and Flow Out. Flow-in sensors are located at the mud pump inlets and the flowout sensor locations are determined according to the rig design. Centralised server uses data from the MezurX sensors as well as third party data providers using WITSML (Wellsite Information Transfer Specification Markup Language is an industry standard that uses a binary file format for transferring wellsite drilling data), OPC (Open Platform Communication is a software interface standard that allows Windows programs to communicate with hardware devices), and other protocols. This detection system considers drilling activity variations while performing alarm calculations, and logs data for subsequent review, analysis and reporting. Audio and visual alarms and multiple preconfigured displays are utilized to provide key data for decision making. (Details can be seen at: http://www.mezurx.com/fads.php)

X-OMEGA System: This system uses a pressure sensor for measuring flow and density of fluids during drilling operations. It provides data for early kick and fluid anomaly (kick or loss) detection in both water and oil based muds, and critical mud weight monitoring, hole cleaning, barite sag detection and other measurements.

It can also be used in conjunction with MezurX's FADS system for comprehensive early kick detection. 
Flow and density is determined from pressure sensors located in a branch of the flow line, combining the principles of a conventional wedge meter and hydrostatic pressures.

These pressure sensors offers to provide the features that overcomes the shortcomings of Coriolis systems. Such as it can maintain the accuracy even in the presence of substantial levels of cuttings and/or gas. Sensor's large internal passageways accommodate high flow rates without blockage. This sensor does not require cleaning or flushing and it doesn't have any moving parts. This sensor is compact in size, less intrusive, and lower installation cost. (Details can be seen at: http://www.mezurx.com/fads.php).

\subsubsection{Detection of Wellbore Influx and Loss through Pressures}

A method to detect the kick and loss, as well as other wellbore anomalies, using the standpipe pressure (SPP) and annulus discharge pressure (ADP) was proposed [16]. The behavior of the SPP and ADP and the comparison of these pressures with each other are useful in identifying deteriorating conditions in the down-hole. Pressure sensors are smaller and easier to install than Coriolis flow meters. To detect the kicks and losses, the warning alarms are set based on pressure change equivalent to total flow or continuous total change in volume. Other drilling problems such as washout and plugging are also detected based on changes in pressure. To reduce noise and make interpretation easier, variance is normalized $[16,17]$.

This method is comparable with the use of a Coriolis flow meter. The results showed in that study are comparable for the time used for detection, as well as the flow and volumes. This method also allows for detection of anomalies with a shut-in well, which is not 
possible with a conventional flow meter. Plugging and vibration do not affect it as this kick detection method relies on pressure measurements as opposed to flow meters. This system is also proposed for managed pressure drilling operations $[16,17]$.

\subsubsection{Detection of Kick and Loss Incidents using PWD/LWD Tools}

The PWD service provides the bottom-hole pressure measurements. The PWD tool consists of high-accuracy quartz pressure gauges that measure annular and bore pressure. The PWD data have a number of valuable applications. Specific applications related to the influx and loss detection include $[18,19]$ :

- down-hole measurement of Equivalent Circulating Density (ECD).

- kick detection, including shallow water flows.

- swab/surge pressure monitoring while tripping and reaming.

- measurement of hydrostatic pressure and effective mud weight in the down-hole.

- Leak-Off Test (LOT) and Formation Integrity Test (FIT) data without circulating to condition the mud.

PWD sensors enhance drilling efficiency by delivering crucial, real-time bottom-hole pressure measurements. It gives the possibility of making faster and better well control decisions [20]. 
The PWD tool kit measures accurate annular pressure, internal pressure, and temperature measurements. The measurements taken down-hole are transmitted through one of the telemetry systems: positive mud pulse, negative mud pulse, electromagnetic, and wired drill pipe telemetry. When the mud is circulating, pressure data can be transmitted in real time and recorded in down-hole memory for later recovery. When the pumps are off and drilling fluid is not circulating, the minimum, maximum, and average pressures are recorded in down-hole memory and when circulation recommences the data is transmitted via mud pulse telemetry. These measurements are very useful to avoid lost circulation and detect kick at its initial stages. The risk of problems that occur due to the unexpected fracture or collapse, also get reduced with the PWD sensor. Most often increase in annular pressure results in ineffective cuttings removal and poor hole cleaning, which can lead to lost circulation. The PWD sensor detects the changes in annular pressure, which allows the appropriate actions to be taken to prevent any event that may lead to well control incident evolving into a blowout. Real-time information helps to maintain wellbore pressures between safe operating limits to avoid kick. The PWD sensor can also detect the influx and loss very promptly. During deep-water riser less drilling, an increase in annular pressure suggests the likelihood of a shallow water flow, while a drop in the annular pressure may indicate gas, oil, and water kicks. Since the sensor is making its measurement down-hole, it is possible to detect pressure changes earlier than traditional surface measurements. The PWD sensor has high-accuracy quartz gauges that measure and record the data. These sensors work with batteries. Bottom-hole pressure measurements could be beneficial in specialized drilling environments, such as, high-pressure/high-temperature, extendedreach, and deep-water wells. When surface and down-hole measurements are compared 
with each other, it is possible to do more accurate and extensive analysis. When a more complete down-hole picture is needed, a command can be sent to the down-hole tools to start measuring high-density data recording and transmission. During LOT (leak-off test) or pumps-off operations the high-density annular pressure data is stored and then transmitted to surface as soon as the circulation is resumed [20].

\subsubsection{Early Kick Detection System Using Numerical Models:}

An early kick detection system using a Bayesian probabilistic model is proposed by Hargreaves et al. [21]. Similarly Kamyab et al. [22] proposed a dynamic neural network model that uses real time data analysis for early kick detection. The model uses real time analysis of mud logging data to detect the kick early. The dynamic wellbore model uses rig data such as pump rate and pipe rotation rate to predict the mudflow out and standpipe pressure. The predicted values are compared with the corresponding measured values from the wellbore. Kick is detected based on deviations between measured data and the model predicted values [23].

A model-based influx detection system is presented in [24]. The model uses two approaches: an ordinary differential equation (ODE) based model and a Partially Differential Equation (PDE) based model. Using an ODE model a globally exponentially stable adaptive observer to estimates the magnitude of the influx or out-flux is presented. Using the PDE model an infinite dimensional observer that detects and quantifies an influx or out-flux is presented [24]. 
To continuously monitor the liquid level in the annulus of wells, the use of an acoustic device installed on the casing valve is proposed [25]. A Gas Kick Warner was developed that measure the propagation time of pressure pulse travelling through the mud system in the well to detect the gas in the wellbore [26]. With the same principle another system was developed. This system can detect gases at early stages, however false alarms due to pipe up and down movement and bit vibrations make it ineffective [27].

A study has been conducted to determine the sensitivity of drilling parameters on early kick detection [28]. A numerical model was also developed to simulate the early kick event and monitor the relative change in the bottom-hole pressure [29]. Experimental study of early kick detection using multiple down-hole parameter monitoring is presented in [30].

\subsection{Permanent down-hole Monitoring Systems:}

Permanent down-hole sensors are installed in the down hole to monitor the pressure, temperature, density, and flow rate. These sensors are used for real time reservoir monitoring during the production operation or well completion. The pressure and temperature sensor monitor the pressure behind casing in the B annulus (Annuli between the production casing and the next outer casing string). The pressure and temperature gauge uses Quartz crystals for long term accurate measurement and Silicon-On-Insulator (SOI) technology that enables the use of these sensors in high temperature environment, significant reduction in size, better shock resistance, and increased data rate $[33,34]$. The down-hole flow sensors can measure multiphase flow including fluid fraction and flow rate from single or multilateral well configuration. These down-hole flow sensors are very compact, so they are installed in short zones, i.e., between the packers and in the casing. 


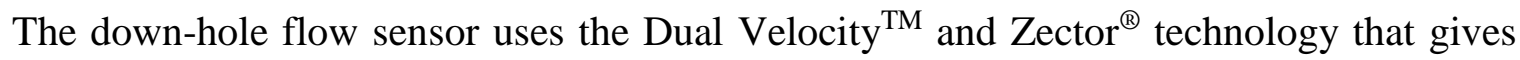
best possible accuracy [33]. They are designed to work in harsh environment (high temperature and pressure) [33].

\subsection{Data Transmission from Down-hole to the Surface}

Transmitting the down-hole measurement data to the surface is a challenge. Four different types of telemetry systems are used in the industry to transmit the down-hole data to the surface. The telemetry converts the measurements taken at the down-hole to a signal, which is compatible with the particular data transmission systems. The most common telemetry techniques are mud pulse telemetry, electromagnetic telemetry, and wired drill pipe telemetry [31].

Mud Pulse Telemetry is a wireless data transmission technique. It transmits the down-hole measurement data to the surface using the pressure pulses through the drilling fluid flowing along the wellbore. The mud pulse systems use valves to modulate the flow of drilling fluid in the bore of the drill string, generating pressure pulses that propagate up the column of fluid inside the drill string and then are detected by pressure transducers at the surface. Mud Pulse Telemetry does not transmit the down-hole measurement data while tripping, cementing, and running casing or executing other well completion operations when the flow of the drilling fluid is stopped. Since Mud Pulse Telemetry uses the drilling mud to transmit the data, it requires un-interrupted fluid flow for data transmission. Statistics show that, in the past $24 \%$ of the well control events during the drilling phase happened while 
tripping operation was in progress. During the completion stage $28 \%$ of the well control incidents occurred while tripping was in progress [31, 32].

Electromagnetic Telemetry is another technique for wireless transmission of down-hole measurement data to the surface. This technique does not use the drilling fluid to transmit the data from the down-hole to the surface, but it uses low-frequency electromagnetic waves that propagate through the earth and are detected by a grounded antenna at the surface. Electromagnetic telemetry is more reliable and gives an opportunity to transmit data at higher data rates than Mud Pulse Telemetry. Electromagnetic telemetry is particularly used when drilling with air, foam or aerated mud. It is used in areas with high mud losses as well as in geothermal drilling. These two telemetries do not offer a bandwidth required to transmit all acquired data from the down-hole. As the distance between surface receiver and the down-hole transmitter increases, the data rate may decrease to a dozen bits per second. The reduced data rate restricts the data transfer in only one direction.

Recently introduced Wired or Networked Drill String proves to be better alternative to the traditional data transmission system. It is a high bandwidth data transmission system that offers data transmission rate 10,000 times faster than current mud pulse telemetry. It also gives an opportunity to transmit in both directions without any interruptions. This technique also gives an opportunity to control the down-hole tools from surface [32].

Majority of the early kick detection systems are based on: exclusive surface parameters and numerical models. As mentioned earlier exclusive surface monitoring has several limitations. Numerical models also uses surface parameters to estimate and predict the 
down-hole condition. Even though down-hole monitoring is more advantageous than surface monitoring, relying only one type of down-hole parameter (pressure) $[12,13]$ may increase the possibility of false alarm due to various problems such as sensor failure. No significant experimental study has been conducted to monitor the relative change in multiple down-hole parameters for early kick detection. 


\section{Chapter 3. Design of a Laboratory Scale Down-hole Assembly of the Drilling Rig Model}

This chapter describes the early kick detection methodology, design and instrumentation of the laboratory scale down-hole sensor assembly of the drilling rig model. The down-hole assembly is equipped with the essential features to simulate the kick events and monitor the down-hole condition in real time.

\subsection{Early Kick Detection Methodology}

The early kick detection methodology basically monitors some of the early indicators of a kick in the bottom of the wellbore.

During an underbalanced condition, formation fluids may start to flow into the wellbore. When the influx starts to enter the wellbore, due to the addition of formation fluid, a change in the down-hole parameters may give accurate and quick information about the anomaly in the down-hole when compared with observation of changes only in surface parameters. This experimental study aims to monitor some of the early kick indicators in the downhole, which can give crucial information to detect the influx at its initial stage. Thus, the down-hole pressure, density, electrical conductivity, and mass flow rate of the fluid in the bottom of the wellbore are monitored to detect the inflow of formation fluids at its earliest phase. These down-hole parameters can subsequently be used to predict the risk of a blowout. 
The down-hole pressure provides an indication of the pressures being imposed on the wellbore, as well as the frictional pressure drop in the down-hole. Variation in the downhole pressure may indicate down-hole problems. A decrease in down-hole fluid density may indicate that a lighter fluid is flowing in the annulus. Monitoring the mass flow rate at the down-hole also provides indication of reservoir influx. A decrease in the electrical conductivity of the fluid indicates the addition of the formation fluids into the wellbore.

Although all mentioned down-hole parameters might provide an indication of formation influx, none rarely provide a definitive answer individually. Therefore, the different downhole parameters are interpreted together in order to understand what is actually happening in the down-hole. These down-hole parameters are also studied individually to evaluate the effectiveness of each indicator in identifying the influx.

In the down-hole of the experimental setup, the pressure sensor is used to monitor downhole pressure, the Coriolis flow meter is used to measure the mass flow rate and density, and the conductivity sensor is used to measure the electrical conductivity of the fluid.

To simulate a kick scenario, compressed air (represent the formation fluid) is injected into the bottom of the wellbore. Similar to Managed Pressure Drilling the pressure in the bottom of the wellbore is adjusted with the backpressure valve. A solenoid valve is used to inject and control the compressed air to simulate various influx events. A gas flow meter measures the amount of influx entering the wellbore. The pressure sensor and the temperature sensor measure the compressed air pressure and temperature. 
To evaluate the various kick scenarios, the pump speed is adjusted and using the backpressure valve the down-hole pressure is maintained at some fixed pressures. The compressed air is injected with higher pressure than the down-hole pressure to represent the underbalanced condition.

Various influx scenarios are simulated on the drilling rig model. During the first set of experiments, the compressed air (represent the formation fluid) is injected keeping constant pressure margin between down-hole pressure and compressed air pressure (formation pore pressure). The first experiment is done with 20 psi down-hole pressure and 40 psi compressed air pressure. The experiment is repeated with a 10 psi increase in down-hole pressure and compressed air pressure. In the second set of experiments, the compressed air is injected, maintaining higher pressure margin (30 psi) between down-hole pressure and compressed air pressure. These two sets of experiments are repeated with another flow rate of the drilling fluid. The experiments are repeated with several kick sizes, i.e. varying the duration of the air injection. The data from all the sensors is logged and recorded using a Data Acquisition System (DAQ) to detect the kick and the data can be used to predict the risk of a blowout. This study evaluates how an influx affect the mass flow rate, density, pressure, and conductivity of the fluid in the down-hole.

This thesis uses imperial units to reflect the practical application of the setup and generated data (as oil and gas industry uses imperial units). The conversion factors for the variables used in this study to SI units are: Density: $1 \mathrm{lb} / \mathrm{ft}^{3}=16.02 \mathrm{~kg} / \mathrm{m}^{3}$; Mass flow rate: $1 \mathrm{lb} / \mathrm{min}$ $=0.454 \mathrm{~kg} / \mathrm{min} ; 1 \mathrm{usgpm}=6.3 \times 10^{-5} \mathrm{~m}^{3} / \mathrm{s}$. Pressure: $1 \mathrm{psi}=6894.76$ pascal . 


\subsection{Structure of Drilling Rig Model}

This section briefly discusses the main structure of the drilling rig model $[35,36]$. The down-hole sensor assembly of the drilling rig model is designed according to the piping and instrumentation diagram shown in Figure 7. The experimental model is constructed at the Drilling Technology laboratory in Memorial University of Newfoundland.

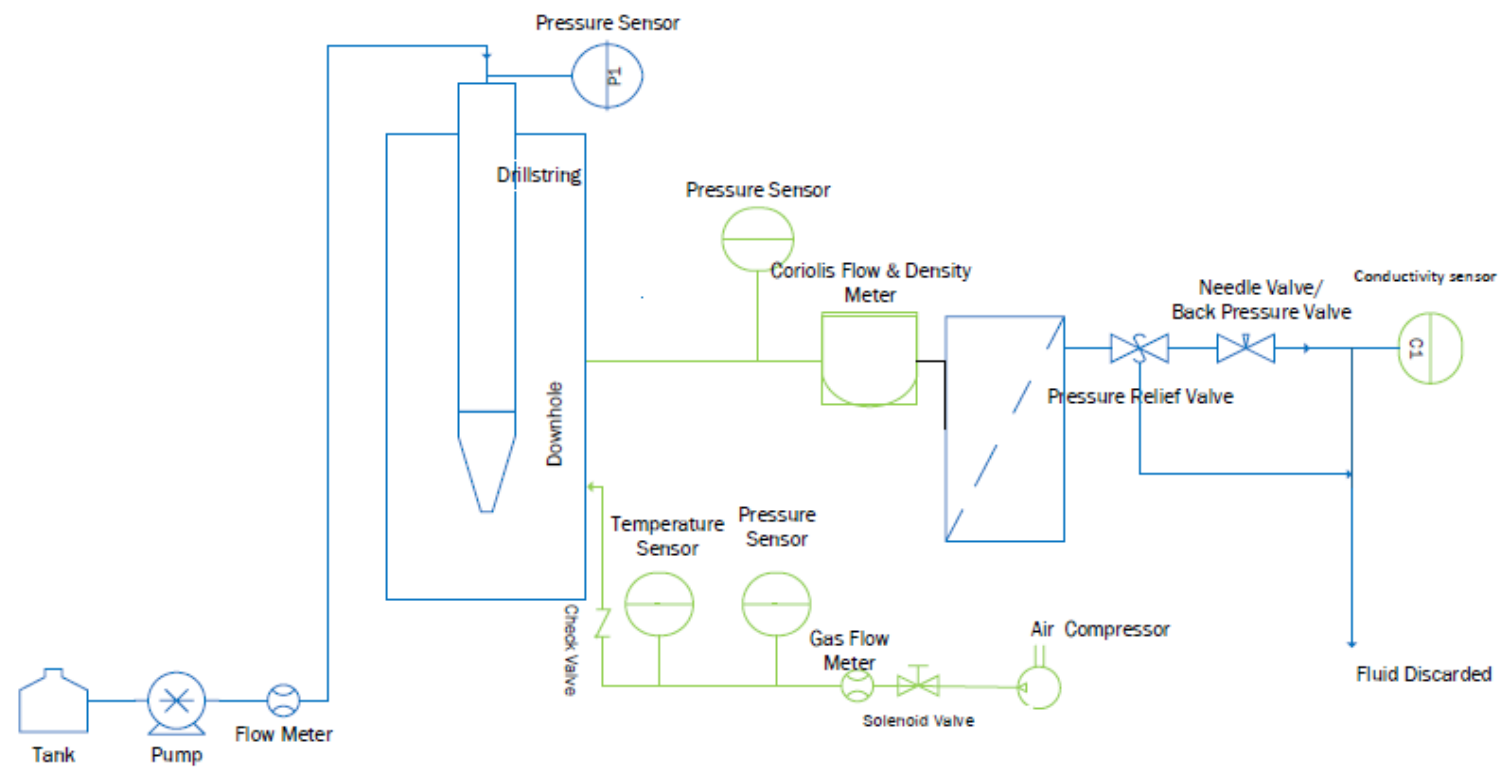

Figure 7. Piping and Instrumentation Diagram of Experimental Model

The steel frames are custom built for mounting the drilling rig setup, the down-hole equipment, and the kick injection setup. The drilling rig experimental model is mounted on the steel frame indicated as frame 1 in Figure 8. Down-hole measurement equipment and kick injection setup are mounted on another steel frame indicated as Frame 2 in Figure 8, 
which is located at the right side of the wellbore. The frame 1 is bolted on the concrete floor whereas the frame 2 is portable. The cylindrical long steel cell (14" in length and 4 " in diameter) represents the wellbore as shown in Figure 8. The lower part of the cell represents the down-hole area, which is extended using a pipe to install the down-hole sensors.

At the top of the wellbore a pipe works as the drill string (DS). The piping of the drilling rig model is 1 " in diameter and it is made of steel. All the piping, bends, and T-fittings are connected together with threaded joints. Two T-fittings are used to mount the pressure sensors, one at the down-hole sensor assembly and another at the kick injection set up. A temperature sensor on the kick injection setup and a conductivity sensor on the down-hole sensor assembly are installed on other two T fittings.

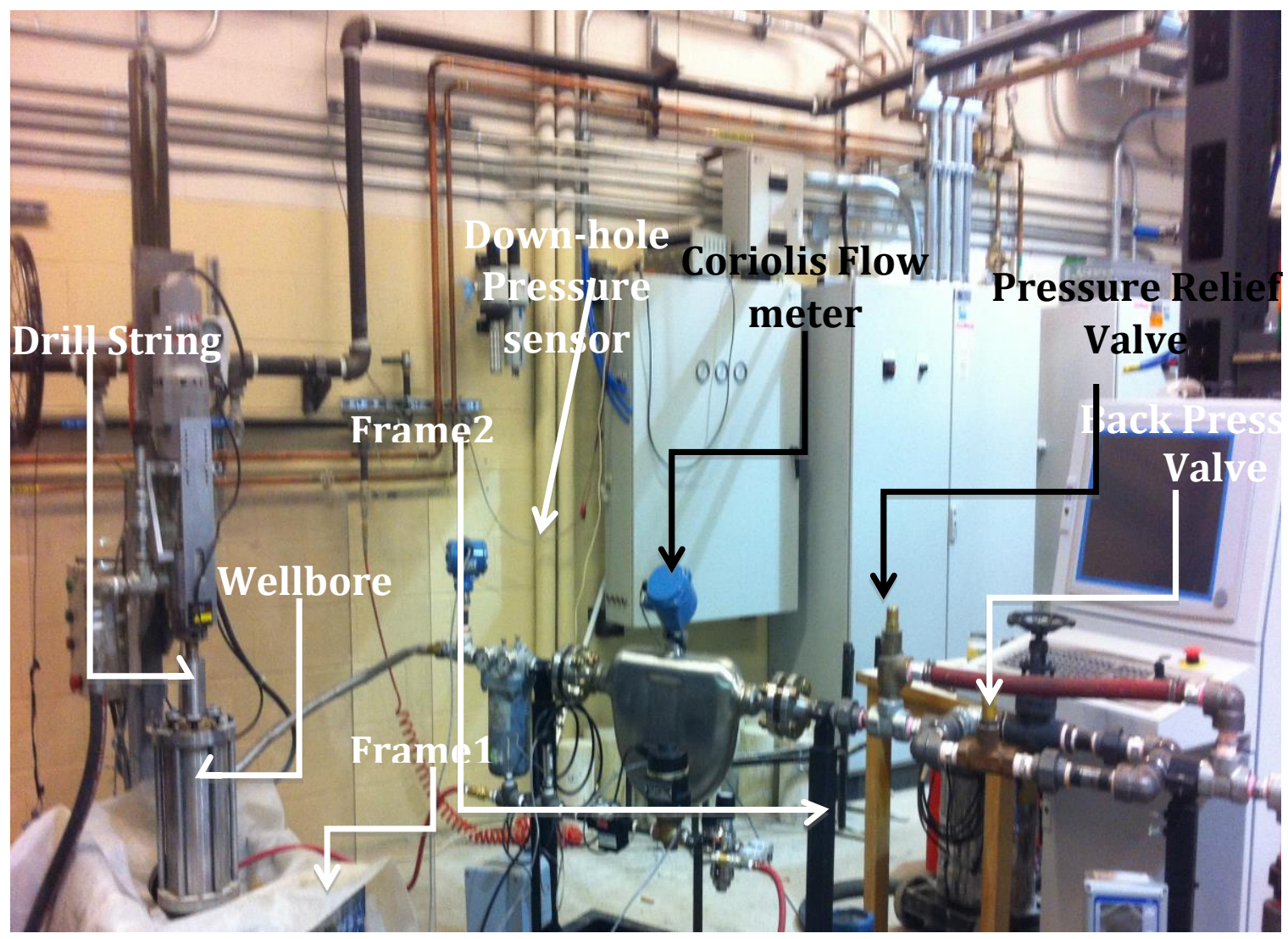




\section{Figure 8. Experimental Setup}

A mud circulation system consists of a mud tank and a positive displacement pump. A hose joins the pump to the drilling apparatus. A positive displacement pump extracts the drilling mud from the mud tank and pumps the drilling mud with a certain amount of pressure to the drilling apparatus $[35,36]$. Drilling mud from the mud tank is pumped into the wellbore through the drill string and the drill bit.

Pressure sensor P1, measures the standpipe pressure (on top of the drill string). To adjust the bottom-hole pressure, the needle valve (back pressure valve) is used as shown in Figure 8. To safely control and divert the high pressure process fluids caused either due to the influx or any other reason, the pressure relief valve is used which is located parallel to the needle valve. The filter acts like a shale shaker that filters out the drill cuttings from the mud $[35,36]$.

To facilitate the early detection of fluid influx from formation, 3 sensors are installed in the bottom of the wellbore. The down-hole sensor assembly includes the pressure sensor that calculates the bottom-hole pressure, the Coriolis flow meter that measures the mass flow rate and the density, and the conductivity sensor that measures the electrical conductivity of the fluid in the down-hole.

As shown in Figure 9, the kick injection setup comprised of a solenoid valve, gas flow meter, pressure sensor, air compressor, pressure regulator, and temperature sensor. Solenoid valve is used to introduce and control the compressed air to simulate different influx scenarios. Gas flow meter and pressure sensor are used to monitor the volume flow rate and the 
pressure of the compressed air (formation gas). The diameter of the piping for the kick injection setup is $1 / 2^{\prime \prime}$.

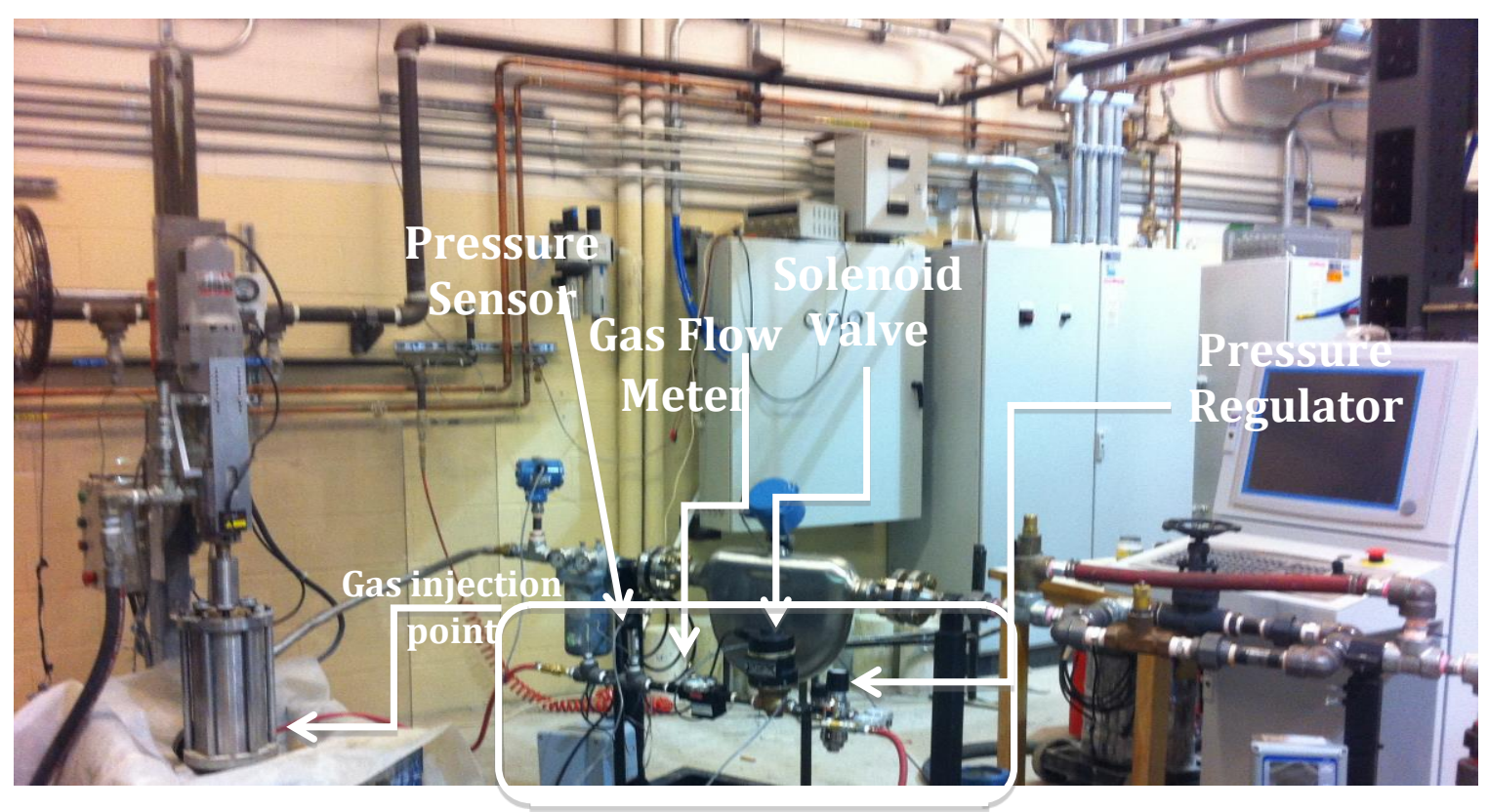

Figure 9. Kick Injection Setup

Process Equipment: This section describes the equipment that are part of the drilling rig model, down-hole assembly, and the kick injection setup. The drilling rig model, downhole sensor assembly, and the kick injection setup are shown in Figure 8 and Figure 9. The subsequent sub-sections briefly describe the equipment installed on down-hole sensor assembly and kick injection setup.

Mud Tank: The mud tank is used to stock the drilling mud. The mud tank supplies the drilling fluid, which is water during this experimental study. The mud tank is located at a height of 4 feet. This thesis is not focusing on monitoring surface parameters so, the tank/pit volume is not observed. The used mud is not recycled so during the experiments the new 
volume of mud is added to the active system in order to maintain the required volume for circulation.

Pump: A positive displacement pump is used to pump the drilling mud. Provided the mud tank is continuously filled with the new mud, it will displace the mud through the system at the preset flow rate. Increasing the pump rate will increase the flow rate at which the mud is flowing out of the active mud tank or pit. A positive displacement pump delivers the constant flow rate at a given speed regardless of discharge pressure. The pump can pump with the flow rate between 5 and 40 usgpm. The maximum pressure that this pump can create is 1000 psi. The pressure relief valve is installed in the downstream to protect the pump and the drilling rig model. The positive displacement pump continues to produce the consistent flow rate and pressure regardless of the pressure in the downstream. Since, positive displacement pump does not have shut off head like a centrifugal pump, it continues to generate the set flow rate until the process pipeline ruptures or the pump gets damaged severely. Therefore a pressure relief valve is used to protect the equipment from being exposed to high pressure which is beyond their design limits.

Valves: Four kinds of valves are used on the experimental setup. Location of these valves is shown in Figure 8 and Figure 9.

Pressure Relief Valve: To protect the equipment located in the bottom of the wellbore the pressure relief valve is used. To restrict the down-hole pressure, the increased pressure is released using a pressure relief valve by diverting the pressurized fluid to flow from the bipass line out of the drilling rig setup. The pressure relief valve opens at a fixed set pressure (250 psi) to protect the down-hole equipment from being subjected to pressures that go 
beyond their design limits. The pressure sensor can hold the pressures up to $2000 \mathrm{psi}$ and the flanges of Coriolis flow and density meter can withstand 1360 psi pressure, and the conductivity sensor can only handle up to $250 \mathrm{psi}$. The outlet of the pressure relief valve is open to atmosphere with a $2 "$ pipeline.

Solenoid valve: The solenoid valve in the kick injection setup is used to inject the compressed air into the bottom of the wellbore. It is a normally closed solenoid valve. It can handle a maximum of $500 \mathrm{psi}$ pressure and the operating temperature range is $-15^{\circ} \mathrm{C}$ to $100^{\circ} \mathrm{C}$. The bronze solenoid valve was selected because it offers fast switching and compatible with the timer.

Check Valve: The check valve (non-return valve/one way valve) is a valve that allows the fluid to flow in one direction and prevents the process flow in the reverse direction. Check valve is mounted on the kick injection setup that can be seen in Figure 7. It is located at the kick injection point to throttle any fluid trying to flow in the reverse direction, i.e., it allows the air flow from compressor into the down-hole but not in the opposite direction. The maximum pressure that it can withstand at $21^{\circ} \mathrm{C}$ is 2000 psi. The operating temperature for this valve is between $-2^{\circ} \mathrm{C}$ to $82^{\circ} \mathrm{C}$. This check valve has a spring close design that helps to prevent slamming. It needs 1 psi cracking pressure. The cracking pressure is the minimum upstream pressure required to operate the valve.

Needle Valve: The needle valve is a backpressure valve, which is used to modify or adjust the pressure in the down-hole. During the experimental study the down-hole pressure is maintained between 20 and 100 psi. 


\subsection{Down-hole Sensor Assembly}

To monitor the various fluid properties in the bottom of the wellbore, different kinds of sensors are installed in the bottom of the wellbore. As shown in Figure 10Figure 10, the down-hole sensor assembly include Coriolis flow and density meter, the pressure sensor, and the conductivity sensor.

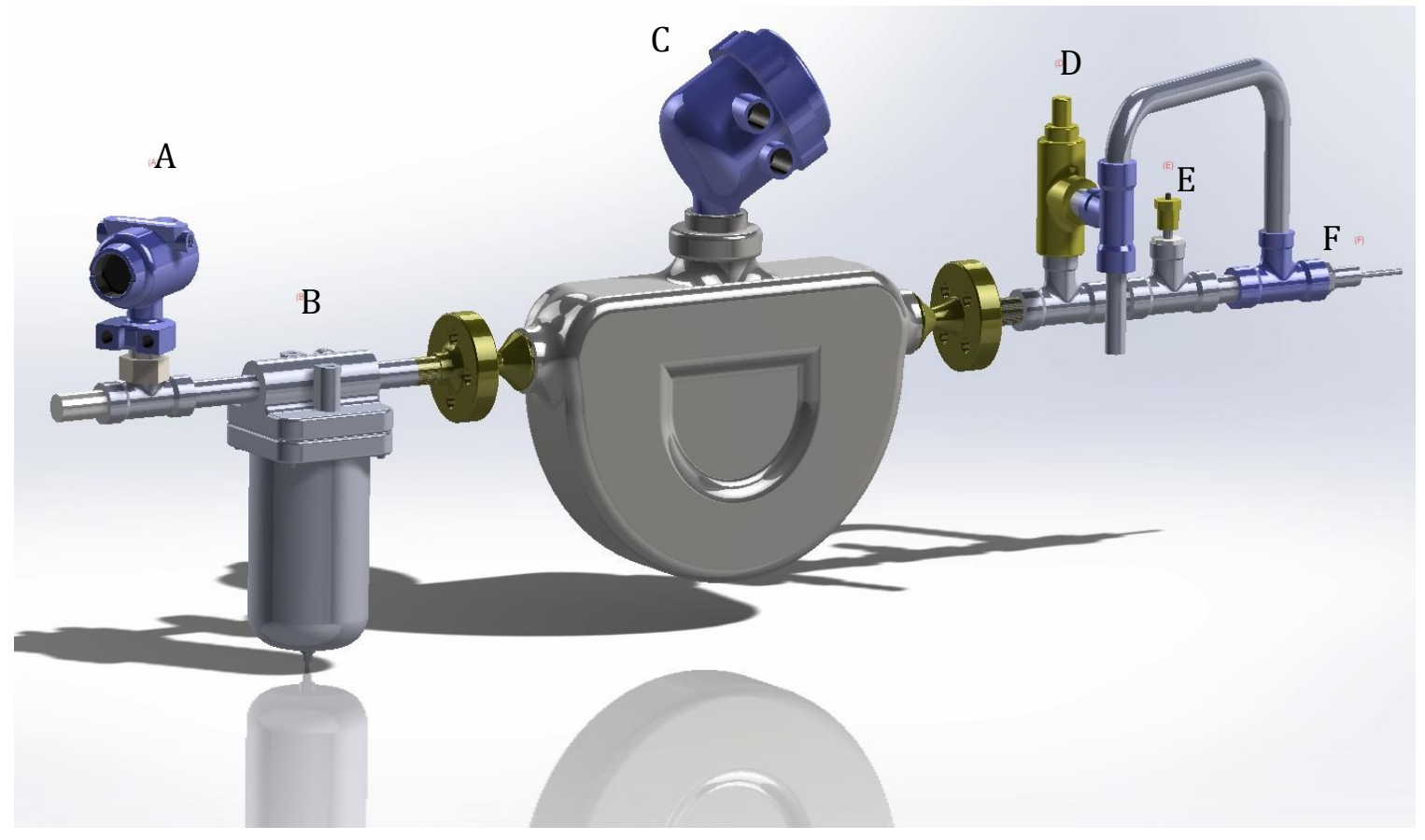

Figure 10. Down-hole Sensor Assembly

A: Pressure Sensor, B: Filter, C: Coriolis Flow Meter, D: Pressure Relief Valve, E: Back Pressure Valve, F: Conductivity Sensor. 


\subsubsection{Coriolis Flow Meter}

For monitoring mass flow rate and density of the fluid in the down-hole, a Coriolis flow and density meter is installed.

This meter is selected because it gives good measurement sensitivity and stability especially when the measuring fluid is two-phase. This flow meter simultaneously measures three process variables (density, flow rate, and temperature). However, the temperature measurement was not acquired during the experiment. This Coriolis flow meter offers good performance on the liquid mass flow, volume flow, and density measurements of single phase as well as two-phase fluids.

This Coriolis flow meter uses a very low frequency Coriolis sensor. So, the two-phase fluid mixture in the measuring tube vibrates with very low frequency, which significantly reduces entrained gas (air) contributions.

\subsubsection{Pressure Sensor}

To measure the down-hole pressure, a smart gauge pressure transmitter is installed on the down-hole sensor assembly. The operating pressure range of this transmitter is 0-800psi, which is within the operating pressure range. Overpressure limit for this pressure sensor is $1600 \mathrm{psi}$ and the burst pressure limit is $11000 \mathrm{psi}$. 


\subsubsection{Conductivity Sensor}

For measuring the electrolytic conductivity of drilling fluid (mud), screw-in low conductivity sensor is installed on the down-hole sensor assembly. It is a contacting type of conductivity sensor; it consists of concentric titanium electrodes separated by a peek insulator. EPDM O-rings seal the internal parts of the sensor from the process fluid. Electrolytic conductivity is a strong function of temperature, so the conductivity readings are typically converted to a value at a reference temperature. This sensor has a built in platinum RTD (Resistance Temperature Detector) to measure temperature.

The conductivity of the drilling fluid (which is tap water) is between $50-200 \mu \mathrm{S} / \mathrm{cm}$. Based on the conductivity range of the fluid being used during the experimentation the cell constant 0.1 is selected. For the conductivity range $0-2000 \mu \mathrm{S} / \mathrm{cm}$, this conductivity sensor gives an accuracy of $0.6 \%$ of reading.

For analyzing the conductivity measurement from the conductivity sensor, a dual inputoutput analyzer from Rosemount is used. It provides the output in the form of 4-20 mA, which is acquired using Data Acquisition System (DAQ). Inputs and outputs of the analyzer are isolated to prevent sensor cross talk, noise, and reduce ground loop error. It is factory calibrated so, it does not require initial calibration at the site. Predetermined cell constant ensures out of the box accuracy. 


\subsection{Kick Injection Setup}

To simulate a kick event, the kick injection setup is designed. The compressed air (formation gas) is injected into the bottom of the wellbore to simulate the kick event. As shown in Figure 11, solenoid valve is used to control and inject the compressed air to simulate several kick scenarios. The gas flow meter, pressure sensor, and temperature sensor are installed to monitor the corresponding physical properties of the compressed air.

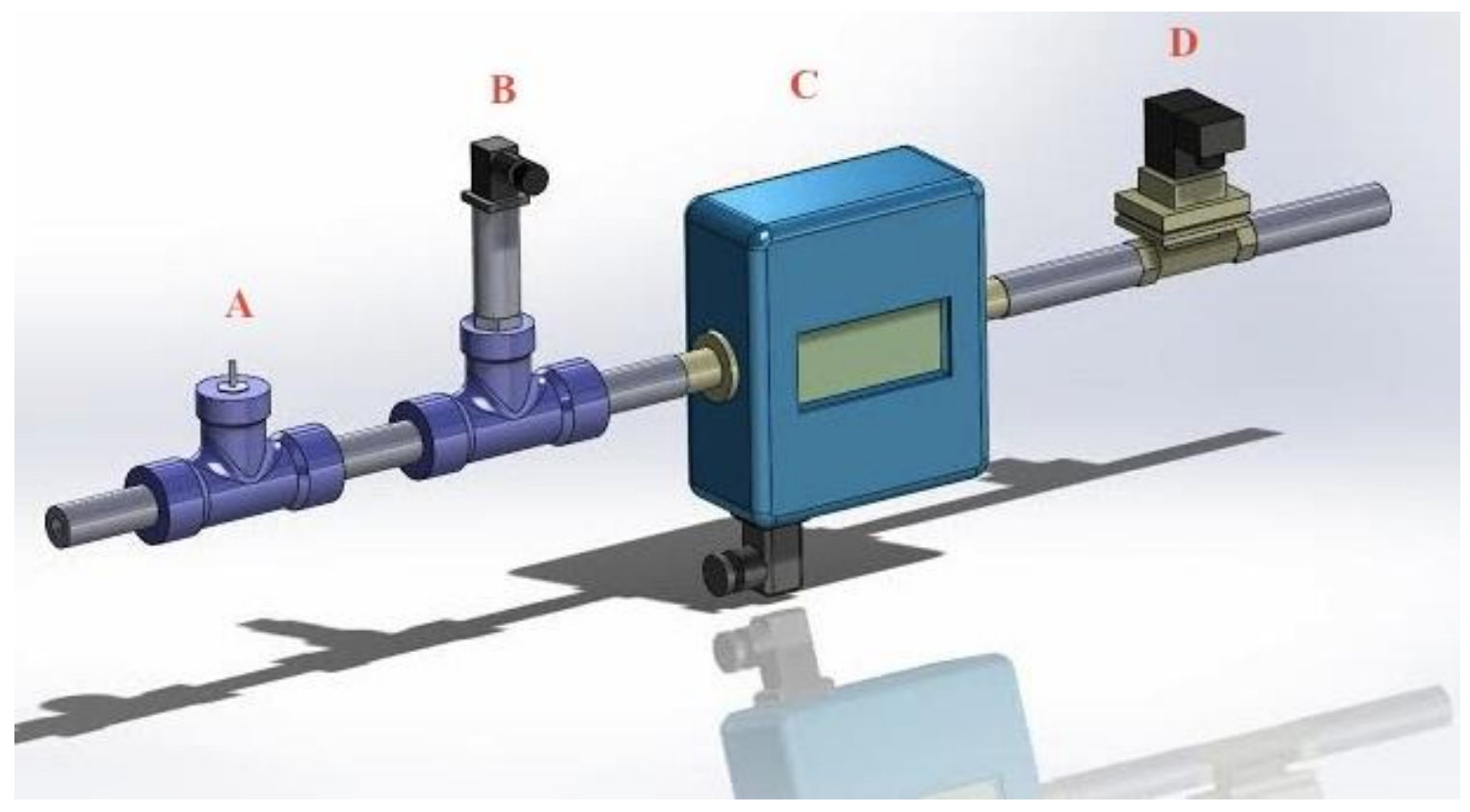

Figure 11. Kick Injection Setup,

A: Temperature Sensor, B: Pressure Sensor, C: Gas Flow Meter, D: Solenoid Valve 


\subsubsection{Air Compressor}

An air compressor from Atlas-Copco is used to inject the compressed air (that represent the formation gas) into the bottom of the wellbore. The air compressor delivers the compressed air with two different fixed pressures with specific flow rates. At 100 psi, compressor delivers air with 25 SCFM (Standard Cubic Feet per Meter) flow rate. At 175 psi compressor delivers the air with 23 SCFM flow rate.

\subsubsection{Pressure Regulator}

To control the pressure of the compressed air, a pressure regulator is used. The air compressor supplies the compressed air with only two fixed pressures, $100 \mathrm{psi}$ and $175 \mathrm{psi}$. However, it is required to regulate it to various pressure values to simulate different kick events. This is a dual stage pressure regulator that provides constant pressure control and delivers continuous consistent pressure regardless of supply pressure variations. To restrict the pressure in the down-hole going beyond the design pressure limit of the drilling rig model, the compressed air is injected with pressures lower than 200 psi. Hence, the regulator is selected for a range of $0-200$ psi. This regulator can take the input pressure up to a maximum of $4000 \mathrm{psi}$ and can reduce the pressure to a range of 0-200 psi. However, the air compressor used in the experimental setup can generate compressed air with a maximum pressure of 175 psi. 


\subsubsection{Temperature Sensor}

The temperature sensor (thermo couple) used on the kick injection setup. The location of the temperature sensor can be seen in Figure 7. At an ambient temperature, it can withstand up to $2500 \mathrm{psi}$ pressure. This high-pressure thermocouple is ideal for pressurized lines or systems. Probe temperature range is $650^{\circ} \mathrm{C}$. Calibration option " $\mathrm{T}$ " is selected because of its accuracy, which is $1^{\circ} \mathrm{C}(0.75 \%)$ for the temperature range $0^{\circ} \mathrm{C}-350^{\circ} \mathrm{C}$.

For logging the temperature data, the temperature transmitter is used. The transmitter offers 2 channels of configurable inputs and accepts inputs from thermocouple (TC) and RTD (Resistance Temperature Detector) with auto reference junction compensation. It gives two isolated current outputs (4-20 mA). It is just 0.89 inches wide and mounted on a DIN rail.

\subsubsection{Gas Flow Meter}

The volume flow rate of the compressed air is measured using a high-pressure gas flow meter. Compressed air flow rate measurements are utilized to estimate the quantity of the compressed air injected into the wellbore. Volume flow rate and the time duration of the influx are used to measure the influx size.

The gas flow meter can tolerate pressures up to $3,000 \mathrm{psi}$ at $21^{\circ} \mathrm{C}$ temperature. The large dial face of the flow meter makes it possible to watch the air volume flow rate readings. The gas flow meter has the flexibility to be mounted at any angle. For air media the temperature range is $-40^{\circ} \mathrm{C}$ to $65^{\circ} \mathrm{C}$. The volume flow rate readings measured by this gas flow meter are $+/-5 \%$ accurate. The volume flow rate range for the gas flow meter is $0-50$ 
SCFM. This range is selected because the flow rate of the compressed air produced by the compressor is in the range of 0-25 (SCFM).

\subsubsection{Pressure Sensor}

The compressed air pressure is measured using the pressure sensor. The working pressure range for the sensor is 0-500 psi. It offers an accuracy of 0.08\% BSL (includes linearity, hysteresis, and repeatability). Operating temperature is $-45^{\circ} \mathrm{C}$ to $115^{\circ} \mathrm{C}$.

\subsection{Data Acquisition System (DAQ)}

The DAQ used in the setup consists of mainly two parts, a power supply unit and a DAQ unit. The power supply unit provides power to the DAQ unit [37].

DAQ system has NI9188 Chassis built in, a NI9237, and a NI9205 for acquiring the data from sensors.

Differential measurement configuration is used to acquire more accurate measurements with less noise. A differential measurement configuration requires two inputs for each measurement, as shown in Figure 12, thus decreasing the number of measuring channels on the NI 9205 to 16 . Table 1, illustrates the signal pairs that are acceptable for differential connection configurations with the NI 9205. So, the DAQ system can acquire the data from 16 sensors. There are 16 plugs, which are available for the sensors to be connected to the DAQ according to their voltage specifications. They are divided into different groups for different voltages. The details of the wiring are shown in Table 1. During the experiments 
six sensors are connected to the DAQ system, three of them (two pressure sensors and gas flow meter) are connected to $24 \mathrm{~V}$ plugs and the other three are connected to $\mathrm{mA}$ passive plugs. Two pressure sensors and gas flow meter are 2 wire devices, it is loop powered, so it is powered from the DAQ, in other words DAQ has to supply excitation voltage to the sensors. Depending on the power requirement the sensors are connected to the respective plugs. In total eight plugs numbered P1, P2, P3, P4, P9, P10, P11, and P12 can acquire the data from $24 \mathrm{~V}$ sensors. In total four plugs numbered P5, P6, P13, and P14 can acquire data from actively powered devices, and another four plugs P7, P8, P15, P16 are not used during the experiment. All three sensor (two pressure sensors and a gas flow meter) used at the setup require $24 \mathrm{~V}$ power supply. So the gas flow meter is connected to plug P4, the downhole pressure is connected to plug $\mathrm{P} 9$, and the compressed air pressure sensor is connected to plug P10. But, Coriolis flow meter and conductivity sensor are 4 wire devices, so the device is externally powered or field powered, thus they do not need $24 \mathrm{~V}$ loop power from the DAQ.

All sensor deliver their output in the form of 4-20 mA. A shunt resister $250 \mathrm{ohm}$ is used for each of these sensors $\mathrm{P} 1, \mathrm{P} 2, \mathrm{P} 3, \mathrm{P} 4, \mathrm{P} 9, \mathrm{P} 10, \mathrm{P} 11$, and $\mathrm{P} 12$, thus providing the sensor data in the form of 2-10 V. Another shunt resistor of value 500 ohms used across plugs P5, P6, $\mathrm{P} 13$, and $\mathrm{P} 14$, thus the data is available in the form of $1-5 \mathrm{~V}$. The real time data of these six sensors are logged using a DAQ and LabVIEW SignalExpress software to monitor the down-hole condition. The data logged from those sensors are used to detect the kick and to assess the risk of a blowout. 


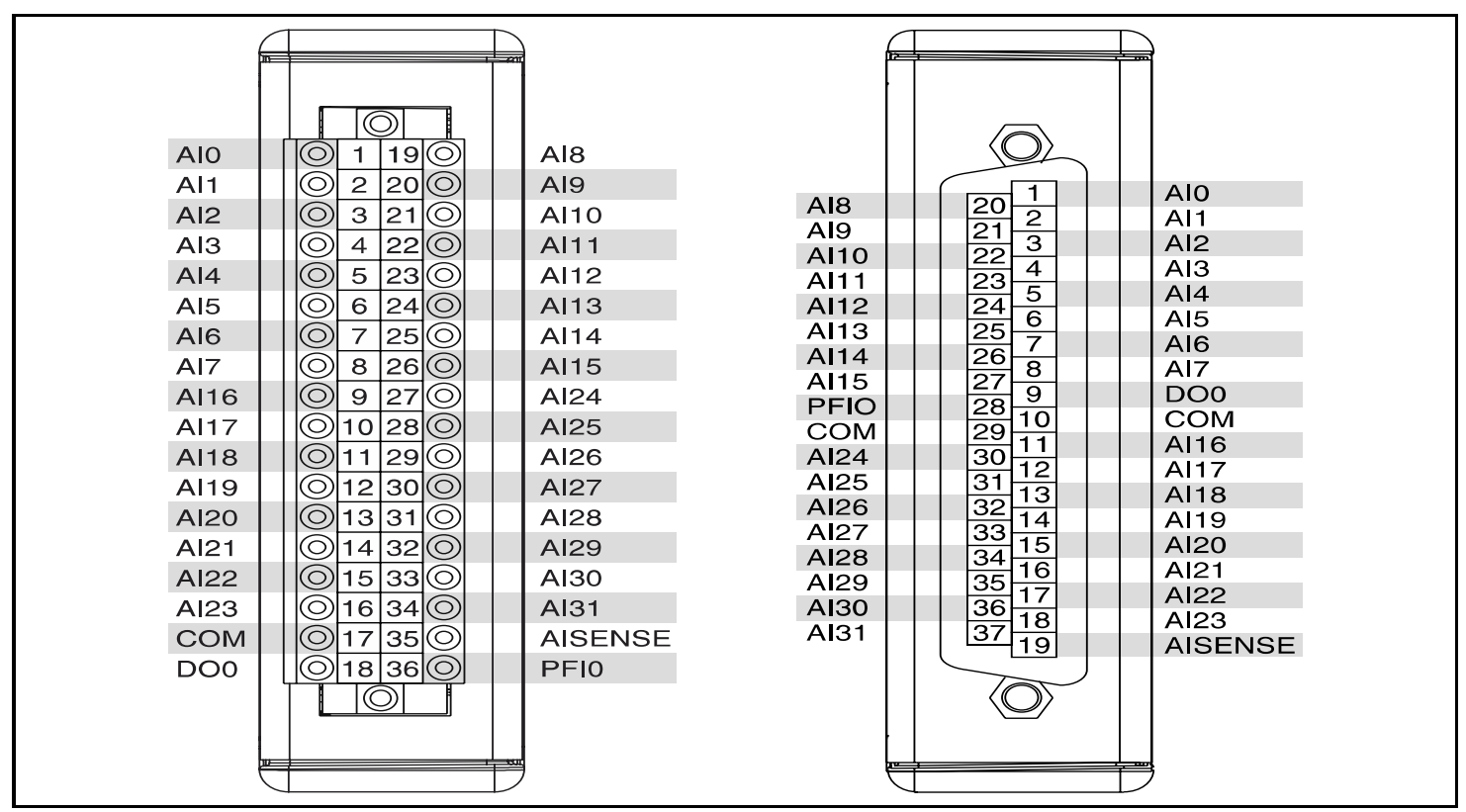

Figure 12. NI 9205 PIN DIAGRAM of 16 Channel Differential Analog Input Module [NI9205 Manual]

Table 1. Differential Channel Pair

\begin{tabular}{|l|l|l|l|l|l|}
\hline Channel & Signal+ & Signal- & Channel & Signal+ & Signal- \\
\hline 0. & AI0 & AI8 & 16. & AI16 & AI24 \\
\hline 1. & AI1 & AI9 & 17. & AI17 & AI25 \\
\hline 2. & AI2 & AI10 & 18. & AI18 & AI26 \\
\hline 3. & AI3 & AI11 & 19. & AI19 & AI27 \\
\hline 4. & AI4 & AI12 & 20. & AI20 & AI28 \\
\hline
\end{tabular}




\begin{tabular}{|l|l|l|l|l|l|}
\hline 5. & AI5 & AI13 & 21. & AI21 & AI29 \\
\hline 6. & AI6 & AI14 & 22. & AI22 & AI30 \\
\hline 7. & AI7 & AI15 & 23. & AI23 & AI31 \\
\hline
\end{tabular}

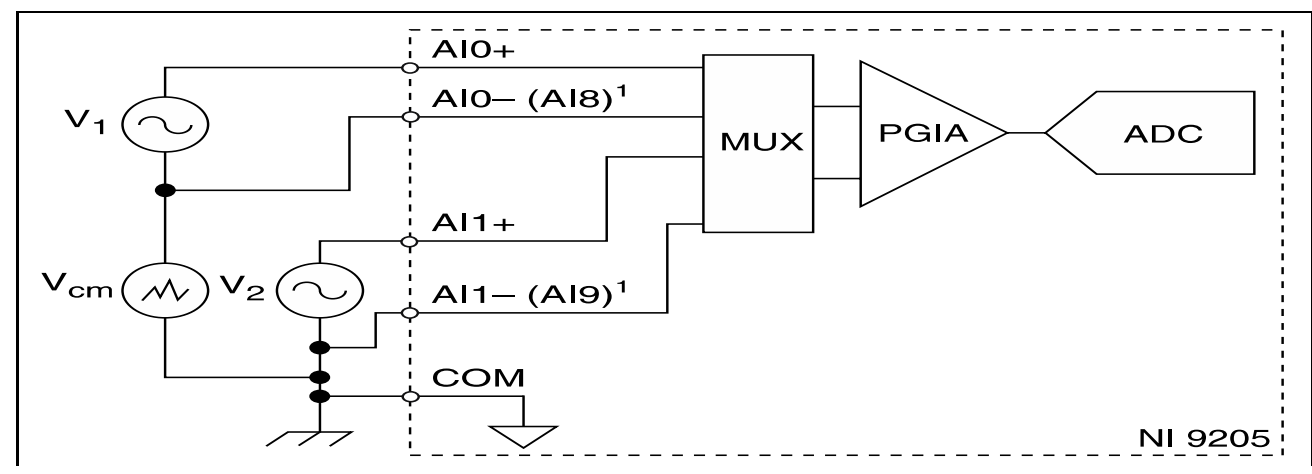

Figure 13. Differential Connection Wiring diagram for one pair of Channels (used for each sensor) [Module [NI-9205 Manual]

As shown in Figure 13, the NI 9205 is connected to the sensors using a differential configuration. In a differential connection configuration, the NI 9205 rejects the commonmode noise voltage, $\mathrm{V}_{\mathrm{cm}}$, during the measurement of $\mathrm{V}_{1}$.

The down-hole sensor assembly of the drilling rig model and kick injection setup were successfully designed. The experimental drilling rig model with the attached down-hole sensor assembly and kick injection setup was successfully tested. The details of the experimental study are presented in the succeeding chapter. 


\section{Chapter 4. Experimental Study}

This chapter presents the purpose of the experiment, methodology to detect the kick event that monitors some of the early indicators of a kick in the bottom of the wellbore. It also

describes the experimental procedure to simulate the kick and detect the kick early using down-hole kick detection system.

\subsection{Purpose of the Experiment}

The aim of the experiment is to observe down-hole conditions through monitoring changes in the down-hole parameters in real time. Monitoring relative changes in several down-hole parameters might enable us to detect the kick early and reliably, and also to gain sufficient insight into the phenomenon to be able to determine conditions in the down-hole that may lead to a blowout.

\subsection{Experiments}

\subsubsection{Down-hole Monitoring Parameters}

1. Down-hole pressure: When the formation fluid with higher pressure enters the wellbore the increase in the downhole pressure would be a clear indication of a kick.

2. Density: When less dense formation fluid enters the wellbore the low density formation displaces the heavier drilling fluid, then it decreases the overall density of the fluid in the system. Therefore, the relative change in the density could be a potential kick indicator. 
3. Electrical conductivity: When the formation fluid with a conductivity different from formation fluid enters the wellbore it changes the conductivity of the fluid in the downhole. So, conductivity variation could be an early kick indicator.

4. Mass flow rate: When drilling fluid is pumped at a constant rate and if formation fluid enters the wellbore, it pushes the drilling fluid to move with high velocity and decreases the mass of the fluid in the system. So the resulting change in the mass flow rate is the key indicator of a kick.

The relative changes in the down-hole pressure, density, electrical conductivity, and mass flow rate of the fluid in the bottom of the wellbore are monitored to detect the kick at its early stage. These parameters are further studied to evaluate the effectiveness of each indicator in identifying the influx, which can subsequently be used to predict the risk of a blowout.

\subsubsection{Control Parameters}

1. Pump rate: $5-40$ usgpm.

2. Down-hole pressure: 20-100 psi.

3. Compressed air pressure: $40-120 \mathrm{psi}$.

Pump Control: The positive displacement pump extracts the drilling mud from the mud tank and pumps the drilling fluid into the drilling string. The pump can build a pressure up to 1000 psi. However, the maximum pressure that is maintained in the down-hole during the experimental study is 100 psi. The pump is controlled with a manual switch. The experiments were conducted with two flow rates: 12 usgpm and 16 usgpm. 
Down-hole Pressure Control: Back Pressure Valve: The pressure in the down-hole is adjusted by using the needle valve, which is a back pressure valve. During the experimental study the back pressure valve is used to maintain the down-hole pressure at $20 \mathrm{psi}$ and up to 100 psi.

Compressed Air Injection Control: The solenoid valve with a timer relay controls the influx injection and the duration of the injection. A plug in timer relay is used to inject the compressed air for a particular known period of time. Influx time is adjusted between 60 and 100 seconds. When $110 \mathrm{~V} \mathrm{AC}$ is applied the relay turns on and it stays on for a set interval of time and then turns off.

\subsubsection{Experimental Procedure}

During the first set of experiments, the down-hole pressure (inside the drill cell) is maintained at 20 psi. The compressed air (gas kick) is injected with a 40 psi pressure. In the down-hole, the relative changes in density, pressure, temperature, conductivity, and mass flow rate are monitored to detect the kick.

The experiment is repeated with a $10 \mathrm{psi}$ increase in the down-hole pressure and compressed air pressure. Throughout the experiments, the down-hole pressure is monitored and adjusted carefully to make sure that the maximum pressure in the down-hole won't exceed the design limit of the down-hole equipment ( $250 \mathrm{psi})$. The experiment set is repeated with a higher pressure margin $(30 \mathrm{psi})$ between down-hole pressure and compressed air pressure. The experiments were conducted with two flow rates: 12 usgpm and 16 usgpm. 


\subsubsection{Experimental Runs}

Experiment set I: Influx (compressed air) is injected while maintaining a constant pressure margin (20 psi) between down-hole pressure and compressed air pressure. Experiments are conducted with a pump rate of 16 usgpm, down-hole pressure $(20,30,40,50,60,70,80$, 90, $100 \mathrm{psi})$, and compressed air pressure (40, 50, 60, 70, 80, 90, 100, 110, 120 psi) (Experimental data is provided in Appendix D).

Experiment set II: Influx (compressed air) is injected while keeping a different pressure margin between down-hole pressure and compressed air pressure. Experiments are conducted with higher-pressure margins (30 psi) between down-hole pressure and compressed air pressure. Experiments are conducted with pump rate 16 usgpm, down-hole pressure $(20,30,40,50,60,70,80,90)$ and compressed air pressure $(50,60,70,80,90$, 100, 110, 120 psi) (Experimental data is provided in Appendix D).

Experiment set III: Experiment sets I and II are repeated with the pump rate of 12 usgpm (Experimental data is provided in Appendix D).

The relative changes in the down hole parameters are monitored to detect the kick early. During each experimental kick scenario four down-hole parameters are monitored. Experiments are conducted by varying three control parameters, i.e., pump rate, down-hole pressure, and compressed air pressure.

The following chapter presents and discusses typical experimental run, which includes base line scenario (steady state condition), influx event, and discussion of the results. 


\section{Chapter 5. Experimental Results}

\subsection{Baseline Scenario}

During a normal operation a relatively steady state condition is observed which is considered as the baseline for all the measured parameters, namely, down-hole pressure, mass flow rate, conductivity, and density as shown in Figure 14. However, during a normal operation, the mass flow rate and the down-hole pressure shows some variations. The reason for the pressure and flow variations are due to the triplex PD pump and nature of the piston operation. A small amount of variation is observed in conductivity readings as well. This variation is due to the fact that the concentration of dissolved salts (salts dissolves into positively and negatively charged ions) present in the tap water will not always be the same.

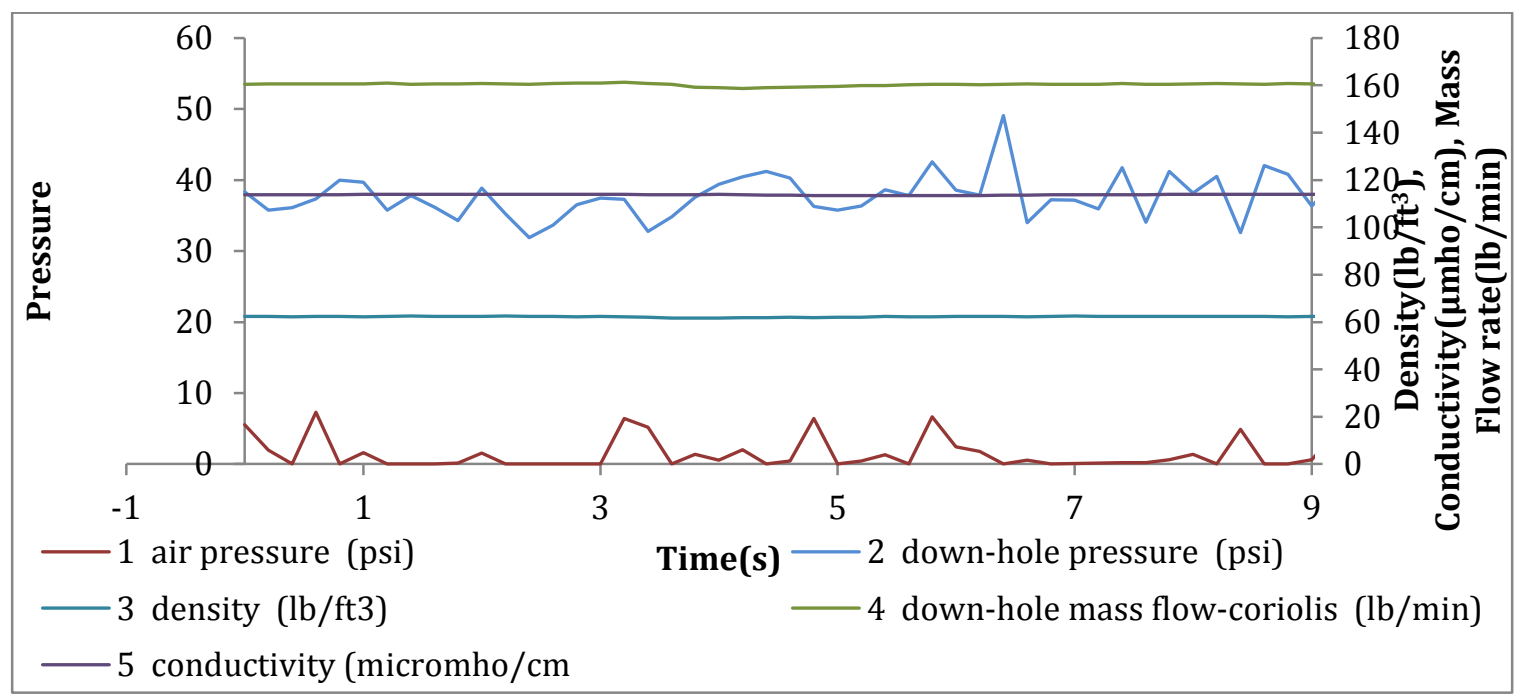

Figure 14. Down-hole Parameter Readings, Baseline Scenario (Steady State Condition) when no Influx occurs. 


\subsection{Influx Scenario}

The start of an influx is characterized by an increase in the down-hole pressure and mass flow rate as shown in Figure 15 and a decrease in the conductivity and density of the fluid in the down-hole as shown in Figure 16.

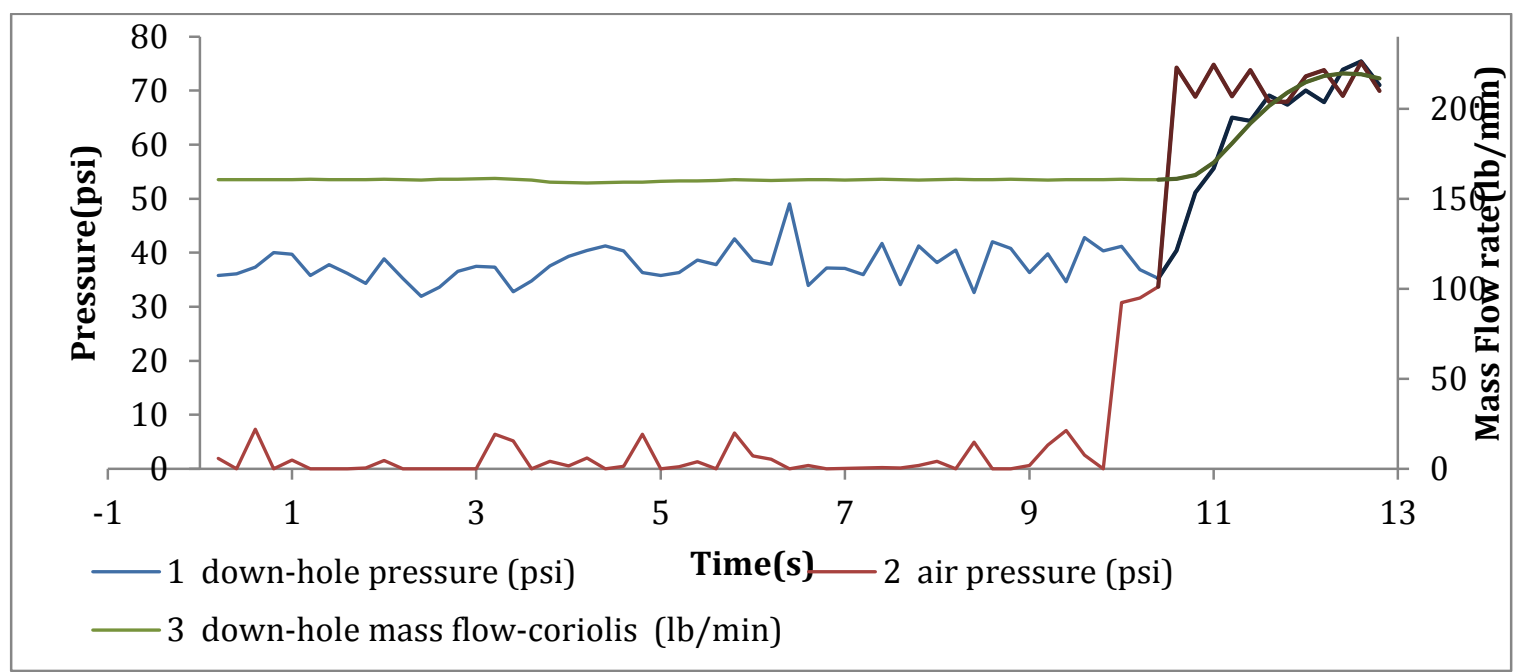

Figure 15. Expected Behaviour of Down-hole Pressure and Mass Flow Rate when Influx Occurs. 


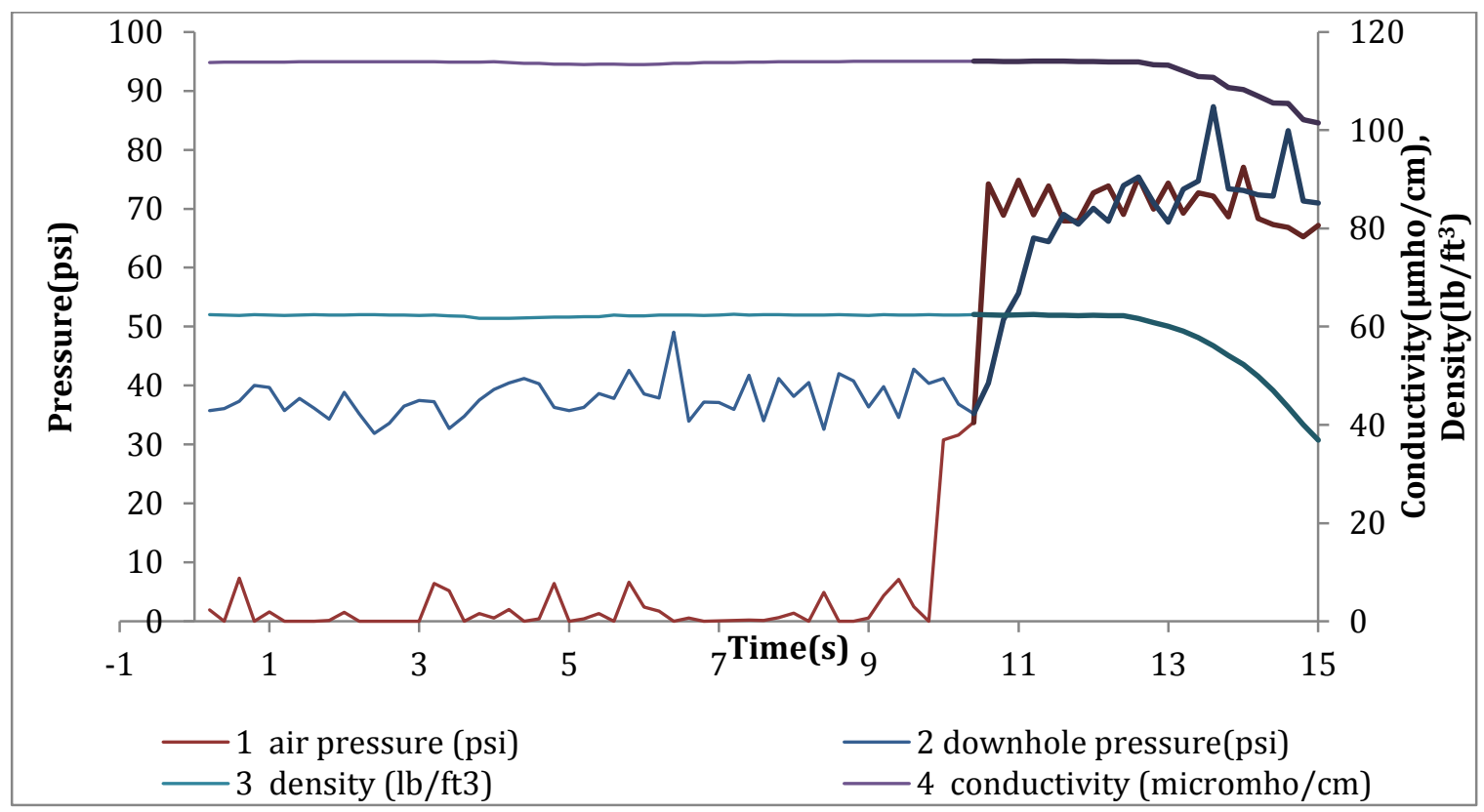

Figure 16. Expected Behaviour of Down-hole Pressure, Conductivity, and Density when Influx Occurs.

Kicks are identified based on the changes in down-hole parameter readings, i.e., the magnitude of the change and the duration of change from the baseline. All down-hole parameters, including pressure, mass flow rate, conductivity, and density trends are compared with the steady state condition (baseline). Deviations from the steady state condition give an indication that down-hole conditions started to deteriorate.

Although any of the above-mentioned down-hole parameters might be used as an indicator of formation influx, they rarely provide a definitive answer by themselves. Thus, the different down-hole parameters are interpreted together in order to understand what is actually happening in the down-hole. Whenever a change in any parameter reading greater than a preselected value (i.e. 15 psi of pressure change, a change of $5 \%$ in mass flow rate, 
density, and conductivity- valid for this particular experimental setup) is observed, other parameters are also considered to check whether corresponding changes are observed in those parameters as well or not (because it might be possible that one parameter gives incorrect reading due to the malfunctioning of the sensor). If at least two parameters show a change in the parameter reading greater than the preselected value consistently for 10 intervals of time each of $1 \mathrm{~s}$ long then it is interpreted that the influx is started. Thus, monitoring the changes in down-hole parameter readings is a good aid in detecting the kick early.

It is concluded that a kick occurred if the following conditions hold. This criteria for kick detection is valid for current experimental condition and setup that is being used during this experimental study:

- At least two of the four observed parameters show variation greater than the threshold limit, i.e., the down-hole pressure changes by more than 15 psi and/or, density and/or, conductivity and/or, the mass flow rate show variation higher than 5\%, and;

- The above anomalies are present for longer than $10 \mathrm{~s}$.

Upon observing anomaly in at least two of the parameters either ((a and b) or (a and c) or (a and d) or (b and c) or (b and d) or (c and d)) consistently for at least $10 \mathrm{~s}$, then it is interpreted that the kick is initiated because the changes observed in more than one parameter reading continuously for $10 \mathrm{~s}$ is most likely being caused due to the addition of the formation fluid in the wellbore. It is highly unlikely that multiple parameters display false readings at the same time continuously for a significant period of time. 


\subsection{Experimental Results Discussion}

This section discusses a typical experimental scenario to explain how proposed methodology works. In this particular kick event, kick occurs with a pressure of 70 psi when the down-hole pressure is 40 psi. During normal operation, before the start of an influx, the down-hole pressure is approximately 40 psi, the mass flow rate is stable at 160 $\mathrm{lb} / \mathrm{min}$ (when the pump rate is $16 \mathrm{usgpm}$ ), conductivity is about $113 \mu \mathrm{mho} / \mathrm{cm}$, and density is around $62 \mathrm{lb} / \mathrm{ft}^{3}$ as shown in Figure 17, Figure 18, Figure 19, and Table 2). The air pressure increased greater than the down-hole pressure at $11 \mathrm{~s}$ creating an underbalanced condition that force the compressed air to enter the wellbore for the duration of $67 \mathrm{~s}$ and at $78 \mathrm{~s}$ the air pressure decreased to a value lower than the down-hole pressure reverting back to an overbalanced condition. The air injection was initiated at $11 \mathrm{~s}$ and stopped at $78 \mathrm{~s}$ (can be seen in Table 2). The down-hole data curves are highlighted during the influx duration in Figure 17, Figure 18, and Figure 19.

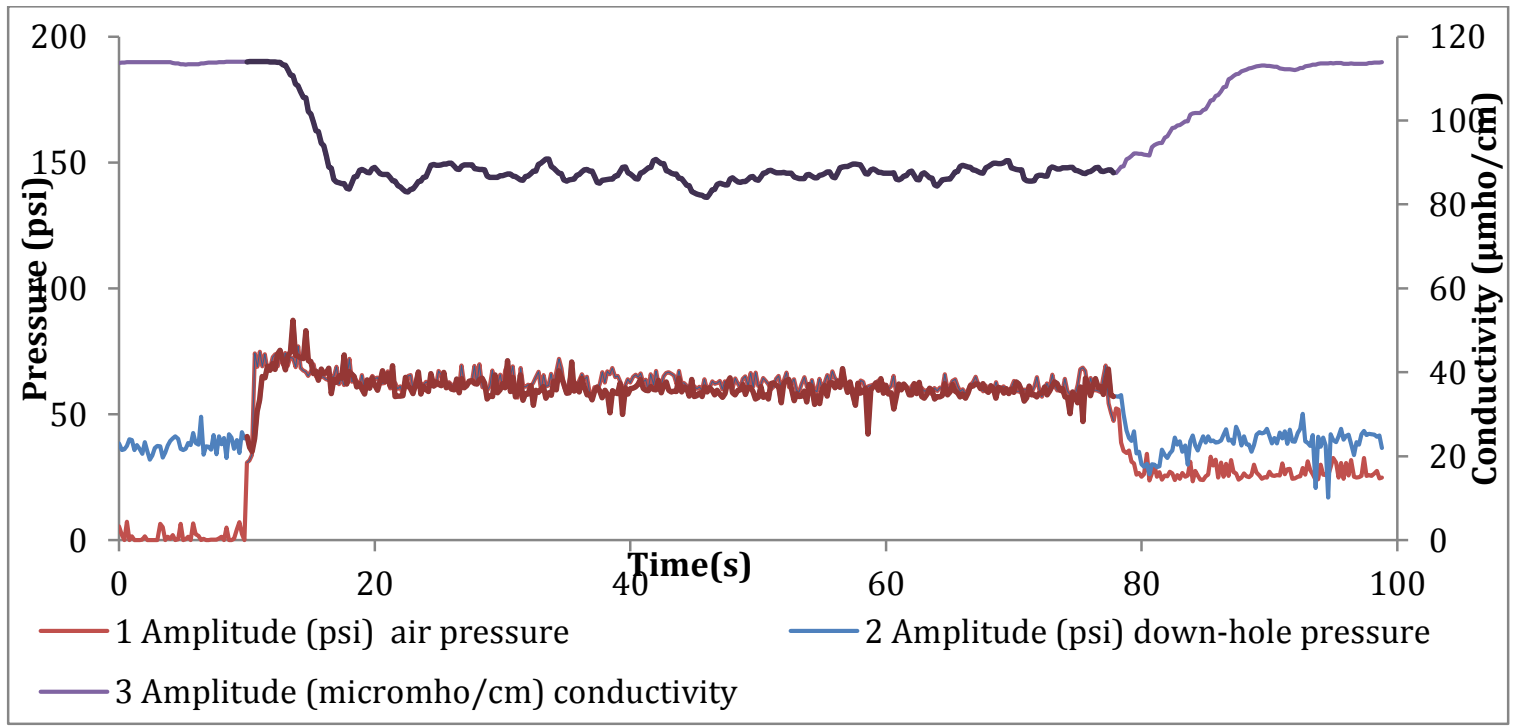


Figure 17. Conductivity Variations in the Down-hole During Influx

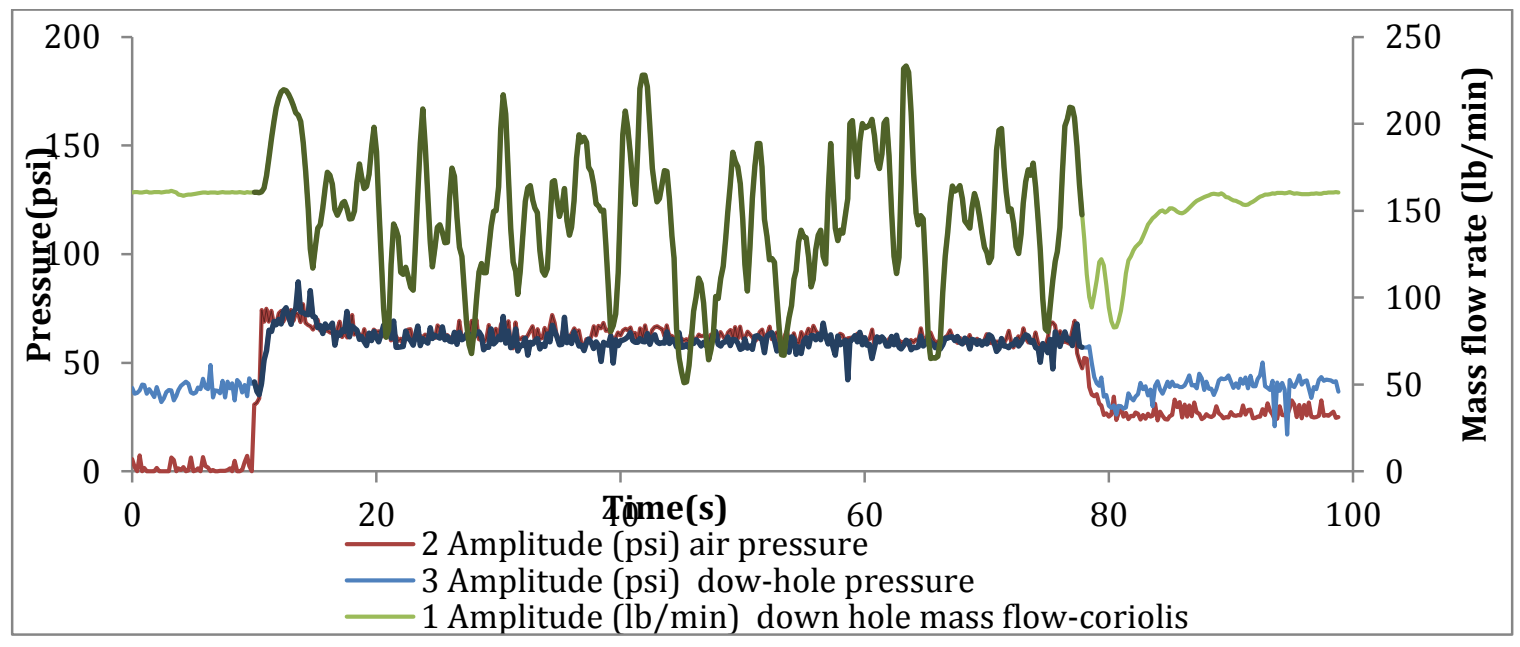

Figure 18. Mass Flow rate Variation in the Down-hole During Influx

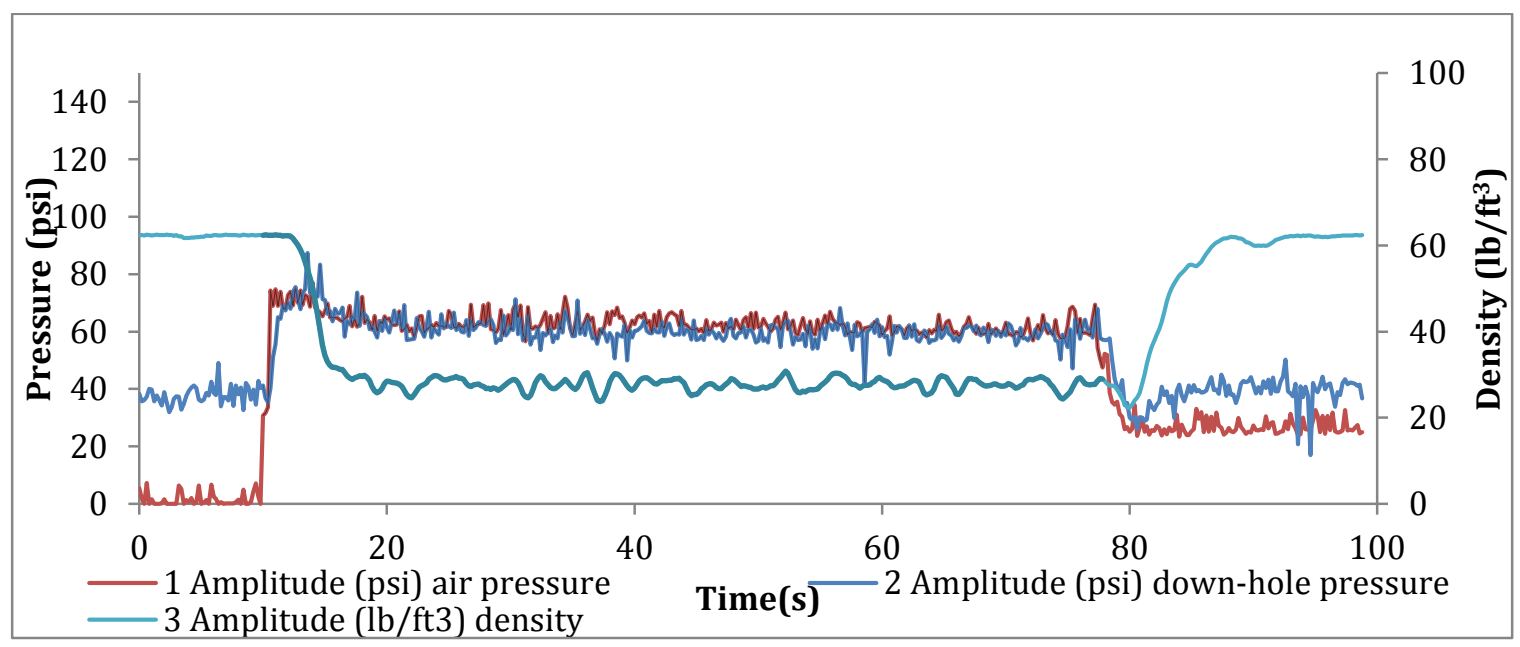

Figure 19. Density Variations in the Down-hole During Influx 
Table 2. Down-hole Parameters Readings

\begin{tabular}{|c|c|c|c|c|c|}
\hline Time (s) & $\begin{array}{l}\text { Down-hole } \\
\text { Pressure } \\
\text { (psi) }\end{array}$ & $\begin{array}{l}\text { Density } \\
\left(\mathrm{lb} / \mathrm{ft}^{3}\right)\end{array}$ & $\begin{array}{l}\text { Air } \\
\text { Pressure } \\
\text { (psi) }\end{array}$ & $\begin{array}{l}\text { Conductivity } \\
(\mu \mathrm{mho} / \mathrm{cm})\end{array}$ & $\begin{array}{l}\text { Mass flow } \\
\text { rate } \\
\text { (lb/min) }\end{array}$ \\
\hline 0 & 38.346 & 62.404 & 5.537 & 113.715 & 160.483 \\
\hline 1 & 39.708 & 62.276 & 1.588 & 113.879 & 160.646 \\
\hline 2 & 38.855 & 62.433 & 1.558 & 113.899 & 160.713 \\
\hline 3 & 37.477 & 62.387 & 0 & 113.889 & 160.981 \\
\hline 4 & 39.364 & 61.718 & 0.553 & 113.899 & 159.009 \\
\hline 5 & 35.754 & 62.000 & 0 & 113.438 & 159.593 \\
\hline 6 & 38.576 & 62.358 & 2.409 & 113.356 & 160.378 \\
\hline 8 & 38.149 & 62.338 & 1.393 & 113.889 & 160.579 \\
\hline 10 & 41.169 & 62.318 & 30.797 & 113.989 & 160.649 \\
\hline 11 & 55.642 & 62.466 & 74.804 & 114.002 & 170.132 \\
\hline 11.5 & 67.276 & 62.727 & 68.148 & 114.033 & 196.772 \\
\hline 12 & 70.049 & 62.272 & 72.671 & 113.930 & 214.605 \\
\hline 12.5 & 66.324 & 61.468 & 77.49 & 113.889 & 219.592 \\
\hline 13 & 67.702 & 59.059 & 74.332 & 113.182 & 213.925 \\
\hline 14 & 73.085 & 49.863 & 77.019 & 108.289 & 189.334 \\
\hline 15 & 70.951 & 34.527 & 67.184 & 101.500 & 128.838 \\
\hline 16 & 66.127 & 31.462 & 64.743 & 93.983 & 172.047 \\
\hline 17 & 62.616 & 29.201 & 68.866 & 85.327 & 150.576 \\
\hline 18 & 66.997 & 29.601 & 72.076 & 83.645 & 145.379 \\
\hline 19 & 59.892 & 26.260 & 65.482 & 88.362 & 162.809 \\
\hline 20 & 61.598 & 28.472 & 64.107 & 88.783 & 183.887 \\
\hline 30 & 62.189 & 28.607 & 60.651 & 86.885 & 163.604 \\
\hline 40 & 60.253 & 26.844 & 62.600 & 87.224 & 157.889 \\
\hline 50 & 56.675 & 26.677 & 61.328 & 85.788 & 144.833 \\
\hline 60 & 61.516 & 28.879 & 58.764 & 87.439 & 197.767 \\
\hline 70 & 58.612 & 27.475 & 63.195 & 88.506 & 125.899 \\
\hline
\end{tabular}




\begin{tabular}{|l|l|l|l|l|l|}
\hline 80 & 30.109 & 22.398 & 25.125 & 92.013 & 94.263 \\
\hline 85 & 38.822 & 55.538 & 25.310 & 102.69 & 151.399 \\
\hline 90 & 41.333 & 60.018 & 27.238 & 113.017 & 156.741 \\
\hline 95 & 39.807 & 62.020 & 32.643 & 113.612 & 160.340 \\
\hline 96 & 41.481 & 61.934 & 30.592 & 113.479 & 159.574 \\
\hline 98 & 42.088 & 62.328 & 25.525 & 113.725 & 160.522 \\
\hline 99 & 36.541 & 62.404 & 28.961 & 113.797 & 160.503 \\
\hline
\end{tabular}

At time $\mathrm{t}=11 \mathrm{~s}$, the influx is initiated, it is observed that the down-hole pressure reading instantly gives the indication of an influx (shown in Figure 17 and Table 2). The mass flow rate also responds very quickly and indicates that the formation fluids started to enter the wellbore (shown in Figure 18). However, the density sensor takes $2 \mathrm{~s}$ (shown in Figure 19) and conductivity sensor takes $3 \mathrm{~s}$ (shown in Figure 17) to indicate the deteriorating condition in the down-hole. The down-hole pressure and the mass flow rate give a quicker indication of a kick than the conductivity and density of the fluid. The delay in displaying the deteriorating well condition by conductivity and density sensor could be because of their position on the sensor assembly and also due to sensor's response time. Overall, all the down-hole parameters give a clear indication of influx within $3 \mathrm{~s}$ as shown in Figure 17, Figure 18, and Figure 19. The changes in the down-hole parameters are greater than the pre-set threshold continuously for $10 \mathrm{~s}$ (can be seen in Table 2), therefore, the influx is confirmed in $10 \mathrm{~s}$.

Due to the small volume of the test rig, the pressure response in the wellbore is very fast, when compared to what would happen in a real well. The pressure in the wellbore is increased in accordance with the gain (shown in Figure 18). When the compressed air is administered/injected with high pressure and velocity, it displaces the drilling fluid 
resulting in a high mass flow rate. Thus, the high mass flow rate observed is due to the increased velocity of the fluid. When air enters the wellbore, the addition of low-density air reduces the mass of the two-phase fluid thus making the mass flow rate low. Due to these reasons, throughout the influx duration extensive variations in the mass flow rate are observed (shown in Figure 18). These variations in the mass flow rate is also due to the fact that the air is not uniformly mixed into the drilling fluid creating air bubbles that might have taken different flow patterns. However, the flow patterns are not studied. When air bubbles are present in the measuring tubes of Coriolis flow meter it shows low mass flow rate and when there are no bubbles, or smaller amounts of air bubbles are present in the Coriolis flow meter measuring tubes, it shows high mass flow rate due to an increase in the velocity of the drilling fluid.

The density of the fluid is measured based on a change in Coriolis flow meter measuring tube's natural frequency relative to the change in mass of the fluid. During the influx when the compressed air with a low mass enters the wellbore the density of the two-phase fluid gets reduced. Due to the presence of non-uniform pattern of two-phase flow, little variation in the density of the two-phase fluid is observed as shown in Figure 19. The density of the two-phase fluid is inversely proportional to the fraction of the compressed air in the wellbore.

When the compressed air starts to enter the wellbore the electrical conductivity of the fluid in the down-hole decreases as shown in Figure 17. The electrical conductivity of a fluid is dependent on the concentration of dissolved salts (salts dissolves into positively and negatively charged ions). Due to the addition of extremely low or negligibly conductive 
air, the conductivity of the two-phase fluid decreases. The conductivity of the two-phase fluid is inversely proportional to the proportion of compressed air in the system. When the influx occurs, due to the non-uniform pattern of the two-phase fluid, a slight fluctuation in the conductivity reading is observed as shown in Figure 17.

When the compressed air injection stops, the mass flow rate starts to decrease to a level lower than the initial mass flow rate as shown in

Figure 20, it is due to the fact that even when the air stops entering the wellbore some amount of air is still present. Due to the presence of low density air the mass of the twophase fluid decreases and the air might also expand. When the gas expands the volume fraction of the air in the two-phase fluid increases. This results in overall decrease in the density of the two-phase fluid. The mass flow rate is defined as: $\mathrm{M}=\mathrm{Q}^{*} \rho\left(\mathrm{kg} / \mathrm{m}^{3 *} \mathrm{~m}^{3} / \mathrm{s}=\right.$ $\mathrm{kg} / \mathrm{s})$, where $\mathrm{Q}$ is the volume flow rate and $\rho$ is the density. Due to the presence of lowdensity air and possible expansion of air a low mass flow rate is observed when compared to the initial mass flow rate, i.e., before the start of an influx as shown in

Figure 20 and Table 2. 


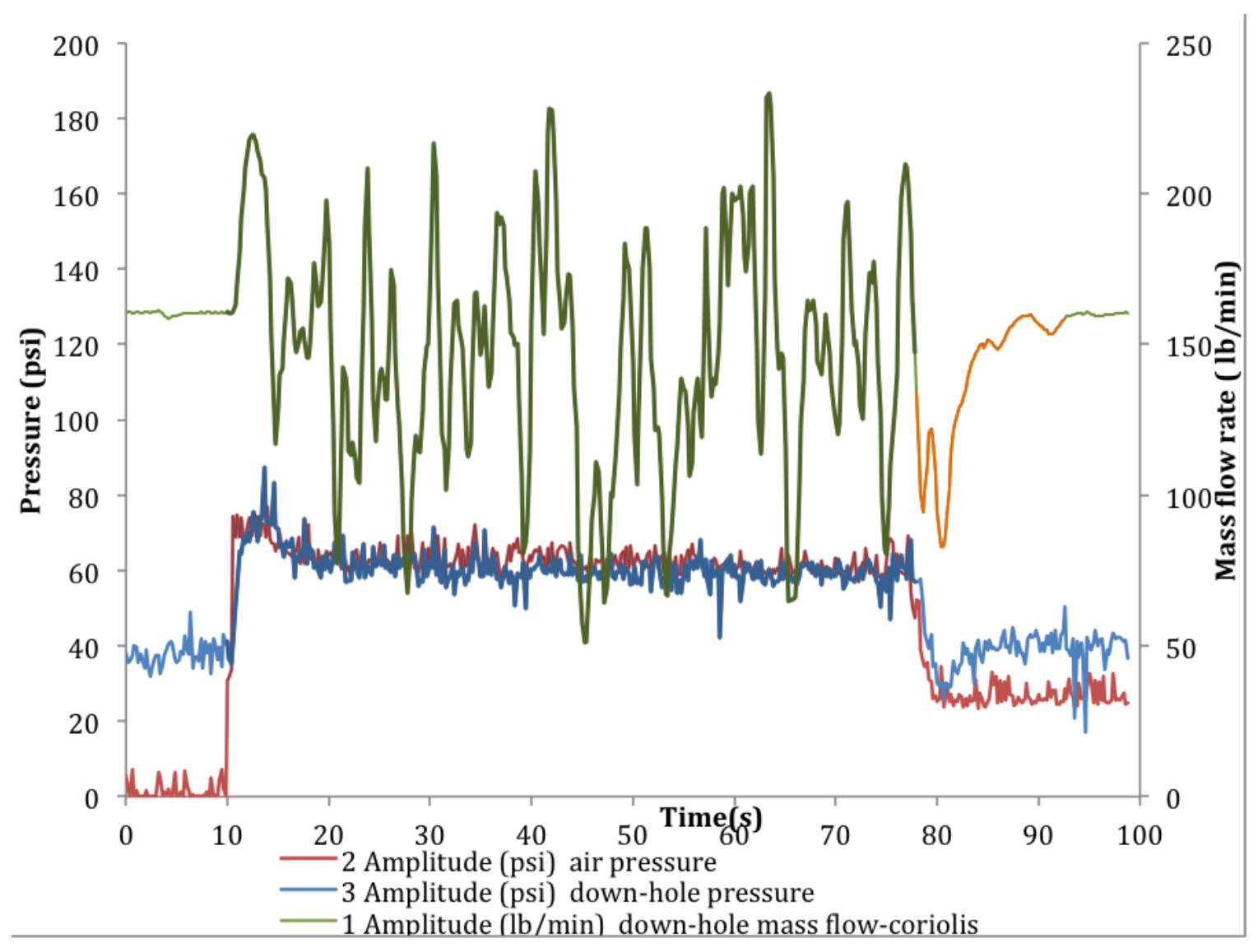

Figure 20. Mass Flow rate after the Influx has stopped 
When the inflow of compressed air stops entering the wellbore the down-hole pressure also decreases to a value that is lower than the initial pressure, i.e., before the start of an influx as shown in Figure 21 and Table 2.

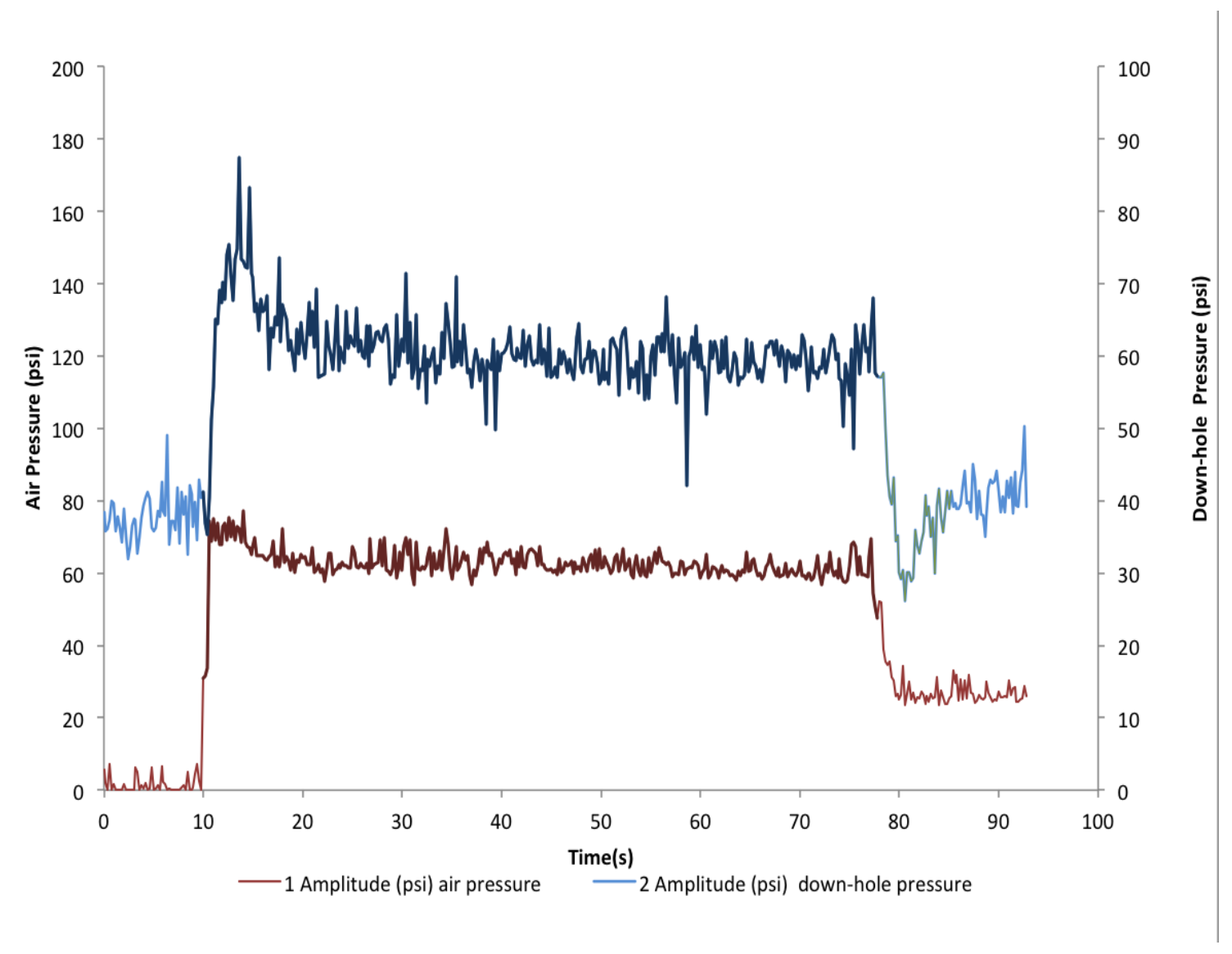

Figure 21. The Down-hole Pressure after the Influx has stopped

The pressure reduction may happen due to the following reasons:

- Frictional Loss: Frictional pressure varies with the velocity of the fluid. Thus, due to the increased velocity of the fluid the frictional pressure loss increases. Frictional pressure is defined as: $P=F / A=f \rho v^{2} / 2$, where $F$ is the friction force, $A$ is the area over which the 
friction force acts, $\rho$ is the density of the fluid, $v$ is the velocity of the fluid, and $f$ is a coefficient called the friction factor.

In a laminar flow, there is a relationship between pressure drop $d P / d x$ and mean velocity: $V_{f}=\left(D^{2} / 32 \mu_{f}\right)(d P / d x)$, where $\mu_{f}$ is dynamic viscosity of liquid.

- Reduction in Density: When the compressed air stops entering the wellbore little amount of air is still present. Due to the presence of the low-density air the overall density of the fluid in the down-hole decreases that results in a lower hydrostatic pressure. Hence, the reduction in the density causes the hydrostatic pressure to decrease to a level lower than the initial value, i.e., before the influx initiation. The pressure exerted by a column of liquid of height $h$ and density $\rho$ is given by the hydrostatic pressure equation: $p=\rho g h$.

- Gas Expansion: Assuming the expansion takes place under constant temperature conditions, i.e., isothermal. The isothermal process with the ideal gas law can be expressed as $P V=$ Constant $\operatorname{or} P_{1} V_{1}=P_{2} V_{2}$, where $V=$ gas volume $\left(\mathrm{m}^{3}, \mathrm{ft}^{3}\right)$ suffix 1 denotes initial conditions and suffix 2 denotes final conditions. Therefore, due to the expansion of the gas, the volume of the gas increases thus decreasing the pressure so that PV remains constant.

The mass flow rate of the compressed air that is injected into the wellbore is dependent on the pressure ratio of upstream and downstream pressure, i.e., compressed air pressure and down-hole pressure. During the particular influx scenario that is discussed in this section (Figure 17, Figure 18, and Figure 19), at the beginning the pressure difference between the down-hole pressure and the air pressure was meeting the criteria for choked flow, i.e., 
$P_{u} / P_{d}=1.93$, where $P_{u}=$ absolute upstream pressure (compressed air pressure) and $P_{d}=$ absolute downstream pressure (down-hole pressure).

Thus, at the beginning choked flow occurs. However, very soon it changes to a non-choked flow. It is due to the fact that the volume of the wellbore is too small and it causes the pressure in the down-hole to increase rapidly. This results in a low-pressure difference between down-hole pressure and the influx pressure (compressed air pressure). Due to these reasons, the varying pressure margin between down-hole pressure and compressed air pressure shows less effect on the amount of air entering the wellbore and subsequently on monitored parameters. However, it is observed that low down-hole pressure allows more compressed air into the wellbore when compared to the high down-hole pressure.

The pump that is used with the test-rig provides a predetermined volume flow rate, and it is not affected by any changes in pressure caused by influx.

The cost associated with the proposed methodology doesn't outweigh the potential benefit that can be achieved through applying the proposed methodology. There is a possibility of installation of conductivity sensor and pressure sensor on the down-hole monitoring systems or on the bottom hole assembly. However the flow and density meter used on the experimental setup cannot be used in the down-hole of the real wellbore because of its large size. The down-hole flow sensors can be installed in the down-hole area, in the casing or in the annulus (between the drill string and casing). However, currently the down-hole multi-phase flow sensors are used during the well completion and production process and presently the down-hole flow sensors for drilling operations are not available. By installing 
flow sensors in the down-hole, i.e., at the beginning of return flow path it can quickly sense the change in the mass of the fluid and detect the presence of any formation fluid in the return drilling fluid. The down-hole flow sensors needs to be multiphase as it has to handle the liquid, gas, and solids (drill cuttings).The proposed methodology can facilitate good results when it is used on the wellbore with Wired Drill Pipe Telemetry [32]. It can be used on any well with other types of telemetries such as mud pulse telemetry but it would not be possible to transmit any abnormality that might occur during the no circulation period. 


\section{Chapter 6. Conclusions and Future Research}

\subsection{Summary}

Early detection of fluid influx from formation is crucial to minimize the possibility of a blowout occurrence. There is a high probability of delay in kick detection, when using an exclusively surface-based kick detection system. The possibility of blowout prevention could be high if the down-hole monitoring is done along with the surface monitoring.

The causes and indicators of a kick were studied to determine the sensitivity of drilling parameters to formation fluids. Few of the early kick detection systems that are in practice in the industry were discussed. Most of the early kick detection systems are based on: exclusive surface parameters and numerical models. Exclusive surface monitoring has several limitations including the delay in kick detection and numerical models also uses surface parameters to estimate and predict the down-hole condition. The early kick detection systems that are based on down-hole monitoring are generally relying on only one type of parameter that is pressure, which may increase the possibility of false alarm due to various problems such as sensor failure.

The down-hole sensor assembly of the drilling rig model and the gas injection setup are successfully developed, which is equipped with the essential features to simulate the kick events and monitor multiple down-hole parameters. The air compressor, pressure regulator, and solenoid valve are installed on the kick injections setup to simulate the kick. Coriolis Flow meter, pressure sensor, and conductivity sensor are employed on down-hole sensor assembly to observe the down-hole condition in real time. 
The experimental setup has been successfully tested by simulating different kick events. The down-hole monitoring parameters namely, pressure, conductivity, density, and mass flow rate were identified as early kick indicators and the sensitivity of these parameters to formation fluid was tested on experimental setup. These observations can assess the occurrence of a kick and associated blowout risk. Monitoring of multiple down-hole parameters has the potential to improve the accuracy of interpretation related to kick occurrence, reduce the number of false alarms, and provide a broad picture of down-hole conditions. A methodology to detect the kick without false alarms is also reported. The outcomes of experiments verify the expected behaviour of the variables during an abnormal well condition. The density and conductivity of the drilling fluid, down-hole pressure, and mass flow rate provides the clear evidence of inflow of formation fluid. The increase in down-hole pressure measurement provides a very quick indication of an influx. The mass flow rate also provides a quick indication of the start of an influx. However, the density sensor takes $2 \mathrm{~s}$ and conductivity sensor takes $3 \mathrm{~s}$ delays to indicate the deteriorating well condition. Experimental results show that combinations of all of the four down-hole parameters measurement give a clear indication of the influx within $3 \mathrm{~s}$. The delay observed by the conductivity sensor to detect the kick could be because of the position of the sensor on the down-hole sensor assembly and could be due to the sensor response time.

The increase in mass flow rate corresponds to the increased velocity of the fluid because of the sudden displacement of water by air influx and the decrease is due to the presence of less dense air bubbles. The pressure response in the down-hole increases in accordance with the compressed air pressure. The density and conductivity are inversely proportional to the 
fraction of the compressed air in the wellbore. The kick can be identified with the proposed down-hole monitoring system even when the wellbore is shut-in and there is no circulation of drilling fluid, which is not possible while monitoring only the flow out rate or pit volume at the surface.

Down-hole monitoring has the potential to improve the kick detection system with a precision that is difficult to achieve with surface detection systems. Thus down-hole monitoring can help in preventing catastrophic events by quickly and reliably monitoring down-hole conditions. This will allow more time for remedial activities and minimize the false alarm thus reducing the amount of non-productive time.

\subsection{Future Research}

In the future experiments the down-hole parameters need to be compared with various surface parameters, such as flow-in and flow-out, fluid properties, pit volume. This comparison can be used to experimentally demonstrate that down-hole monitoring saves the time taken to identify a kick by monitoring multiple kick indicators in the bottom of the wellbore when compared to exclusive surface monitoring.

The experiments can be conducted with water and oil based drilling fluids. Changing the drilling fluid will provide the broader view how kick fluids react to these drilling muds which has different properties. To examine the behaviour of water kick, water can be injected/introduced. 
Temperature of the drilling fluid can also be studied to support the existing indicators. To simulate this scenario on the small scale experimental setup the compressed gas or air needs to be warmed to notice the significant difference in the temperature readings. However, many other factors need to be considered while interpreting the temperature readings.

A numerical model may be developed to simulate range of kick scenarios, which would help to develop more effective well control and blowout prevention strategies. The simulation data should be compared with the data collected from experimental runs to confirm the validity. To minimize the human error and to avoid the necessity of continuously monitoring of the down-hole data the proposed early kick detection system can be upgraded to an automatic early kick detection system. Using LabVIEW, the criteria/condition for kick detection could be defined to trigger an alarm that alert the operator about changing down-hole condition using LED indicators, beep, or more complex alerts such as e-mails or text messages. To automatically detect the kick and to calculate the severity of the kick an Artificial Neural Network or Fuzzy Logic can also be used.

To monitor the pressure, density, conductivity, and mass flow rate, sensors needs to be installed in the down-hole monitoring systems of the real wellbore.

To measures the pressure, temperature, density, conductivity, and flow rate in the downhole the permanent down-hole monitoring system (which is currently being used during production and completion stages) need to be developed for drilling operation. 


\section{References}

[1] Robert, F. Mitchell and Stefan Z. Miska. (2011). Fundamentals of Drilling Engineering. SPE Text book Series No. 12, ISBN: 978-1-55563-207-6, Society of Petroleum Engineers.

[2] Robert, D. Grace. (2003). Blowout and Well Control Handbook. Gulf Professional Publishing, ISBN 0750677082.

[3] Hawker, D. (2001). Abnormal Formation Pressure Analysis. Anca Maria Anistoroae.

[4] Vik, R. B. (2001). Well Control Manual. Equilibria Services Ltd (Publisher).

[5] Orban, J. J., Zanner, K. J., \& Orban, A. E. (1987). New Flowmeters for Kick and Loss Detection during Drilling. In: SPE Annual Technical Conference and Exhibition.

[6] Schafer, D. M., Loeppke, G. E., Glowka, D. A., Scott, D. D., \& Wright, E. K. (1992). An Evaluation of Flowmeters for the Detection of Kicks and Lost Circulation during Drilling. In: IADC/SPE 1992 Drilling Conference.

[7] Steine, O. G., Rommetveit, R., \& Harris, T. W. R. (1995). Full Scale Kick Detection System Testing Relevant for Slim-Hole / HPHT Drilling. In: SPE Annual Technical Conference and Exhibition.

[8] Orban, J. J., \& Zanker, K. J. (1988). Accurate Flow-Out Measurements for Kick Detection, Actual Response to Controlled Gas Influxes. In: IADC/SPE Drilling Conference. 
[9] Kick Detection Servicesby @balance. Available:

http://www.slb.com/ /media/Files/miswaco/product_sheets/mpdubd_kick_detection_ps.p df (last viewed on July 6, 2015).

[10] Speers, J. M., \& Gehrig, G. F. (1987). Delta Flow: An Accurate, Reliable System for Detecting Kicks and Loss of Circulation during Drilling. In: SPE/IADC Drilling Conference.

[11] Hargreaves, D., Jardine, S., \& Jeffryes, B. (2001). Early Kick Detection for Deepwater Drilling: New Probabilistic Methods Applied in the Field. In: SPE Annual Technical Conference and Exhibition.

[12] Ritchie, G. M., Hutin, R., Aldred, W. D., \& Luppens, J. (2008). Development and Testing of a Rig-Based Quick Event Detection System to Mitigate Drilling Risks. In: IADC/SPE Drilling Conference.

[13] Nybo, R., Bjorkevoll, K. S., \& Rommetveit, R. (2008). Spotting a False Alarm. Integrating Experience and Real-Time Analysis with Artificial Intelligence. In: Intelligent Energy Conference and Exhibition.

[14] Weatherford-Early Kick/Loss Detection Services. Available: http://www.weatherford.com/weatherford/groups/web/documents/weatherfordcorp/wft16 4783.pdf. (Last viewed on July 6, 2015) 
[15] Flag Fluid Loss and Gain Detection Services. Schlumberger. Available: https://www.slb.com/ /media/Files/geoservices/brochures/flag_br.pdf (last viewed on July 6, 2015). .

[16] Reitsma, D. (2010). A simplified and highly effective method to identify influx and losses during managed pressure drilling without the use of a Coriolis flow meter, In: SPE/IADC Managed Pressure Drilling and Underbalanced Operations Conference and Exhibition.

[17] Reitsma, D. (2011). Development of an automated system for the rapid detection of drilling anomalies using standpipe and discharge pressure. In: SPE/IADC Drilling Conference and Exhibition.

[18] Pressure-While-Drilling (PWD) Sensor-Optimizing drilling efficiency. Available: http://www.halliburton.com/premium/ss/contents/Data_Sheets/web/H02986.pdf. (Last viewed on July 6, 2015).

[19] Sperry Drilling MWD/LWD Services. Available:

http://www.halliburton.com/premium/ss/contents/Brochures/web/H07333_MWD-

LWD_Services_Overview.pdf. (last viewed on July 6, 2015).

[20] Pressure While Drilling Services (http://www.halliburton.com/enUS/ps/sperry/drilling/measurement-while-drilling/pressure-while-drilling-pwdservices.page (last viewed on July 6, 2015). 
[21] Hargreaves, D., Jardine, S., \& Jeffryes, B. (2001). Early Kick Detection for Deepwater Drilling: New Probabilistic Methods Applied in the Field. Society of Petroleum Engineers.

[22] Kamyab, M., Shadizadeh, S. R., Jazayeri-rad, H., \& Dinarvand, N. (2010). Early Kick Detection Using Real Time Data Analysis with Dynamic Neural Network: A Case Study in Iranian Oil Fields. Society of Petroleum Engineers.

[23] Swanson, B. W., Gardner, A. G., Brown, N. P., \& Murray, P. J. (1997). Slimhole Early Kick Detection by Real-Time Drilling Analysis. Society of Petroleum Engineers.

[24] Hauge, E. (2013). Automatic Kick Detection and Handling in Managed Pressure Drilling Systems, Ph.D thesis, Norwegian University of Science and Technology.

[25] Schubert, J. J., \& Wright, J. C. (1998). Early Kick Detection through Liquid Level Monitoring in the Wellbore. Society of Petroleum Engineers.

[26] Li, X., Guan, C., Sui, X., \& Gangtao. (1998). A New Approach for Early Gas Kick Detection. Society of Petroleum Engineers.

[27] Stokka, S. I., Andersen, J. O., Freyer, J., \& Welde, J. (1993). Gas Kick Warner - An Early Gas Influx Detection Method. Society of Petroleum Engineers.

[28] Ayesha, N., Venkatesan, R., Khan, F. (2014), Monitoring Early Kick Indicators at the Bottom hole for Blowout Prevention, In: OCEANS'14 MTS/IEEE Conference. 
[29] Ayesha, N., Venkatesan, R., Khan, F. (2013), Identification and monitoring of Early Kick Indicators for Blowout prevention, In: The Twenty-second Annual Newfoundland Electrical and Computer Engineering Conference (IEEE Newfoundland and Labrador section).

[30] Ayesha, N., Venkatesan, R., Khan, F. (2015), Monitoring of Down-hole Parameters for Early Kick Detection, Manuscript accepted for publication in Journal of Loss Prevention.

[31] Veeningen, D. (2012). Identify safe drilling margin, detect and distinguish kicks from ballooning and better well control for deep water, through independent down-hole measurement. In: SPE/APPEA International Conference on Health, Safety, and Environment in Oil and Gas Exploration and Production.

[32] Gravdal, J.E., Lorentzen, R.J., Time, R.W. (2010). Wired drill pipe telemetry enables real-time evaluation of kick during Managed pressure drilling. In: SPE Asia Pacific Oil \& Gas Conference and Exhibition.

[33] Permanent down-hole monitoring systems: Retreived from http://www2.emersonprocess.com/enUS/brands/roxar/DownholeApplications/Permanent\%20Downhole\%20Monitoring\%20Sy stems/Pages/RoxardownholeFlowsensorsystem.aspx. (last viewed on July 6, 2015).

[34]https://www.slb.com/ /media/Files/resources/oilfield_review/ors99/win99/pages20_3 3.pdf (last viewed on July 6, 2015). 
[35] Khorshidian, H. (2012). Phenomena affecting penetration mechanisms of polycrytalline diamond compact bits. Master's Thesis. Memorial University of Newfoundland.

[36] Li, H. (2011). Experimental Investigation of the Rate of Penetration of Vibration Assisted Rotary Drilling. Master's Thesis, Memorial University of Newfoundland.

[37] Gao, Q, (2015). Development of Laboratory and Field Drilling Tools to Measure Bit Operating Conditions and Drill String Motions. Master's Thesis, Memorial University of Newfoundland.

[38] User Manual-Micro Motion Elite Coriolis Flow Meter.

[39] User Manual- Rosemount Conductivity Analyzer 1056.

[40] User Manual- Rosemount Pressure Transmitter 2088G.

[41] National Instrument: A Guide to Configuring NI-DAQmx for CompactDAQ-9188 Chassis 


\section{Appendix A Equipment Data Sheets}

\section{A.1. Coriolis Flow Meter Data sheet}

Model Number: CMFS100M329N2BAEDZZ

Micro Motion High Performance Coriolis mass flow \& density sensor for low flows

- Unique design delivers unparalleled measurement sensitivity and stability;

- Best two-phase flow capability for batching, loading, and entrained air applications.

- Designed for maximum drainability;

- Can be mounted directly in the line to reduce installatation costs.

100 Sensor nominal size: 1"

M Sensor material: 316L SST

329 Process connection: 1" ANSI CL300 weld neck raised face flange

N Case option: standard (300-series stainless steel)

2 Electronic interface: 4-wire polyurethane-painted aluminum integral enhanced core processor for remote mount transmitters

B Conduit size: 1/2-inch NPT- no gland

A Approval: CSA (Canada \& USA)

E Quick reference guide and manual: English

D Factory accuracy: $0.1 \%$ of mass flow and $0.0002 \mathrm{~g} / \mathrm{cc}$ density

Z Option: No measurement application software

Z Option: none 


\section{A.2. Micro Motion Field Mount Coriolis Transmitter}

Model Number: 2700R12BBAEZZZPK

Micro Motion field mount coriolis multivariable transmitter model

2700 MVD

$\mathrm{R} \quad$ Mounting: 4-wire remote mount transmitter (incl $10 \mathrm{ft}(3 \mathrm{M})$

4 wire sielded PVC cable

1 Power supply: 18 to 30 VDC \& 85 to 265 VAC; self

switchwing

2 Display: Backlit dual line display for process variables and totalizer reset

B Outputs: Two mA; two configurable IO channels - default configuration

B $\quad 1 / 2$ "-inch NPT conduit connections - no gland

A Approval; CSA (US and Canada)

E Language : English - Quick reference Guide and Manual

Z Software option 1: Flow \& density variables (standard)

$Z \quad$ Software option 2: No software options 2

Z Factory options for standard product options

PK Universal mounting bracket included 


\section{A.3. Pressure Transmitter}

Model Number: 2088G4S22A1B4C6

Smart gage pressure transmitter for direct mounting Rosemount model 2088G

- NEW: Accuracy of $+/-0,075 \%$ of span $(0.065 \%$ optional $)$

- NEW: Stability of $+/-0,1 \%$ of URL for 3 years*

- Configurable using the 375/475 HART interface or AMS (Asset

Management Solutions) software

- NEW Rangeability of 50 to 1

- NEW local operator interface (optional)

4

Ajustable range: -14.7 to $4,000 \mathrm{psi}(-1,01$ to 275,8 bar $)$ / minimum: 80 psig

S Output: 4-20 mA dc with HART protocol

22 Process connection/diaphragm material: 316 stainless steel. Fill fluid: Silicone

A Process connection: $1 / 2 "$ - 14 NPT

1 Conduit thread: $1 / 2$ " - 14 NPT

B4 Universal SST mounting bracket with SST bolts

C6 Approval: CSA (explosion proof and intrinsic safety) 


\section{A.4. Conductivity Sensor Data Sheet}

Model Number: 400-12

ENDURANCE ${ }^{\text {TM }}$ Model 400 Screw-in Low Conductivity Sensors

- Consists of titanium electrodes and a 316 stainless teel 3/4 in.

MNPT process connection.

- Maximum temperature for the standard sensor is $221^{\circ} \mathrm{F}\left(105^{\circ} \mathrm{C}\right)$.

- For temperatures up to $392^{\circ} \mathrm{F}\left(200^{\circ} \mathrm{C}\right)$ a sensor-mounted

\section{A.5. Conductivity Analyzer Data Sheet}

Model Number: 1056-01-20-38-AN

Model 1056 Dual Input/Output Analyzer

- Larger display - large display digits for process variables.

- Easier wiring, removable connectors, replaceable signal boards.

- Polycarbonate enclosure material prevents discoloration in direct sunlight.

- Isolation of inputs and outputs to prevent sensor cross-talk, noise and reduce ground loop error.

- 3 year Warranty!

-01 Power: 115/230 VAC, 50/60 Hz

-20 Measurement 1:Contacting Conductivity

-38 Measurement 2: none

-AN Communication: Analog 


\section{Appendix B Wiring Diagrams}

\section{B.1. The Coriolis flow Meter Wiring:}

The transmitter is installed remotely from the sensor. The transmitter and sensor are connected with a 4-wire cable, power and I/O wiring are connected to the transmitter [38].

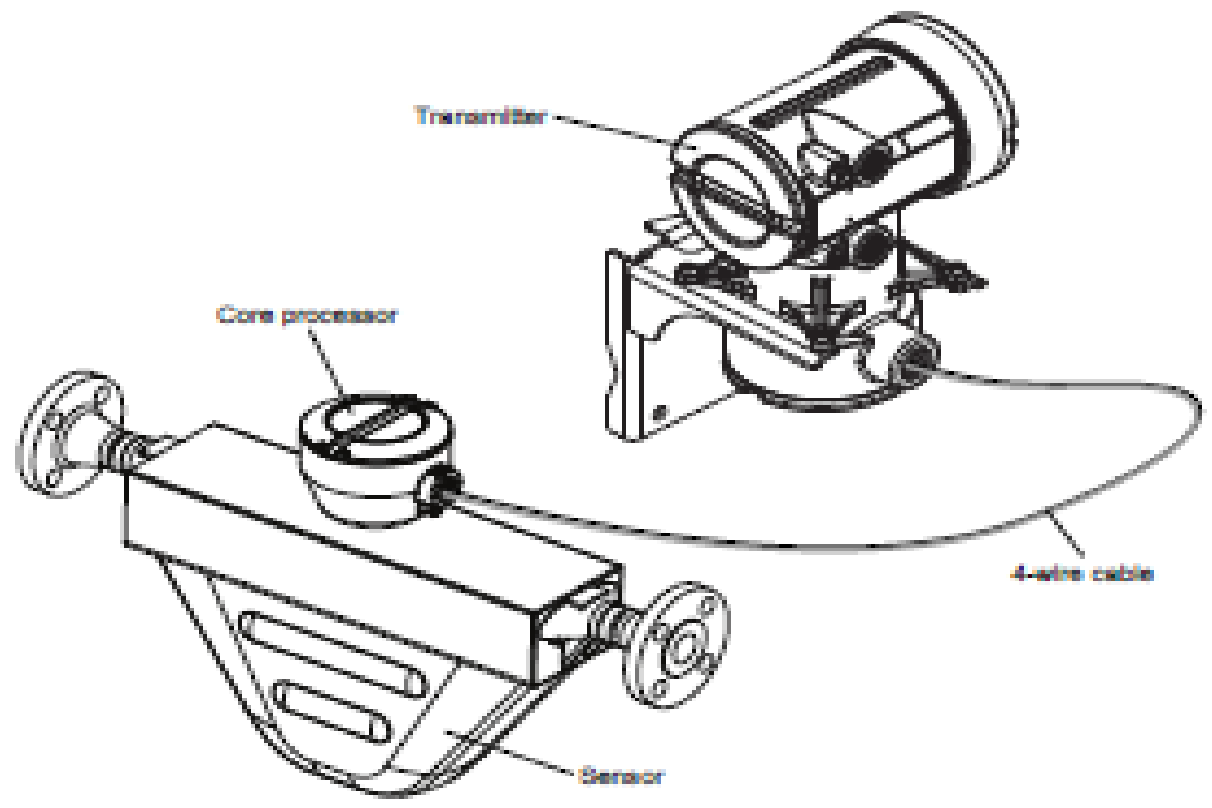

Power supply terminal and ground:

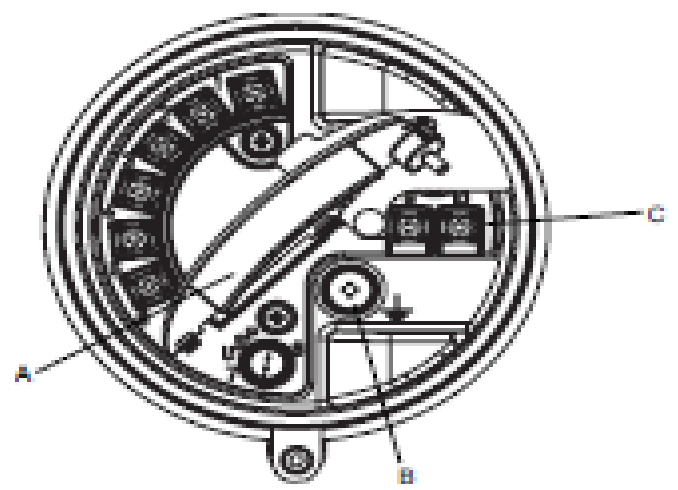

A: Warning flap; B: Equipment ground; C: Power supply wiring terminals 
Input/output wiring terminal:

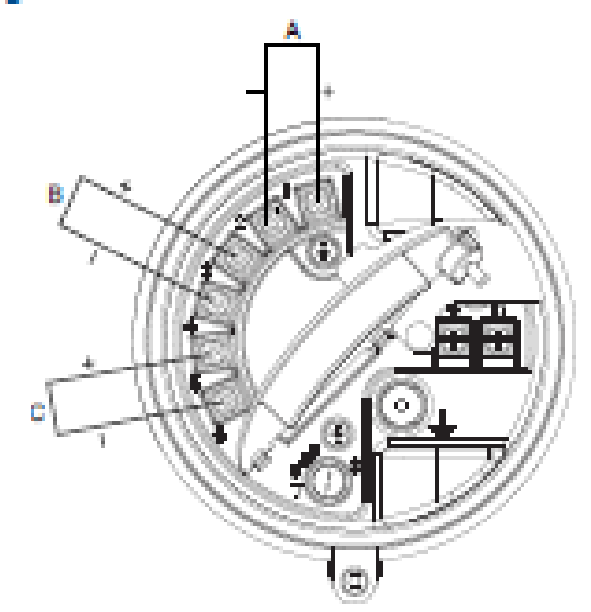

A: mA/HART; B: mA output; C: Frequency output, discrete input.

\section{B.2. Conductivity Sensor Wiring [39]}

Power Wiring:

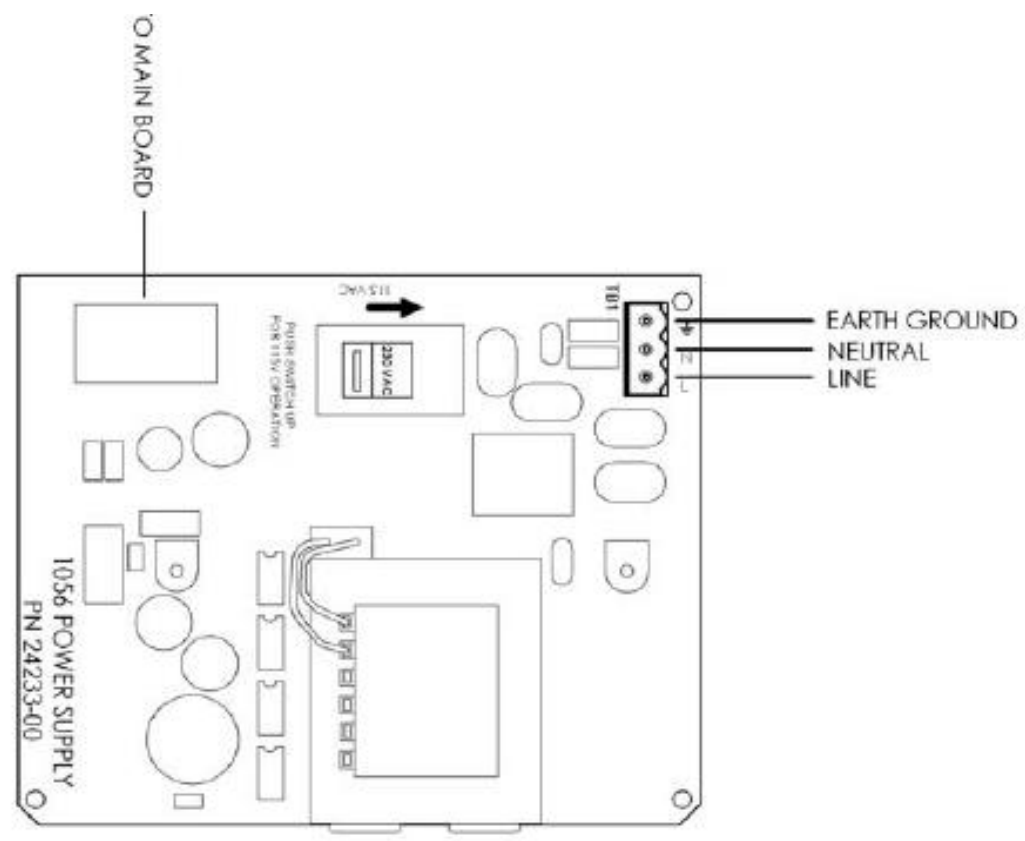


Output wiring:

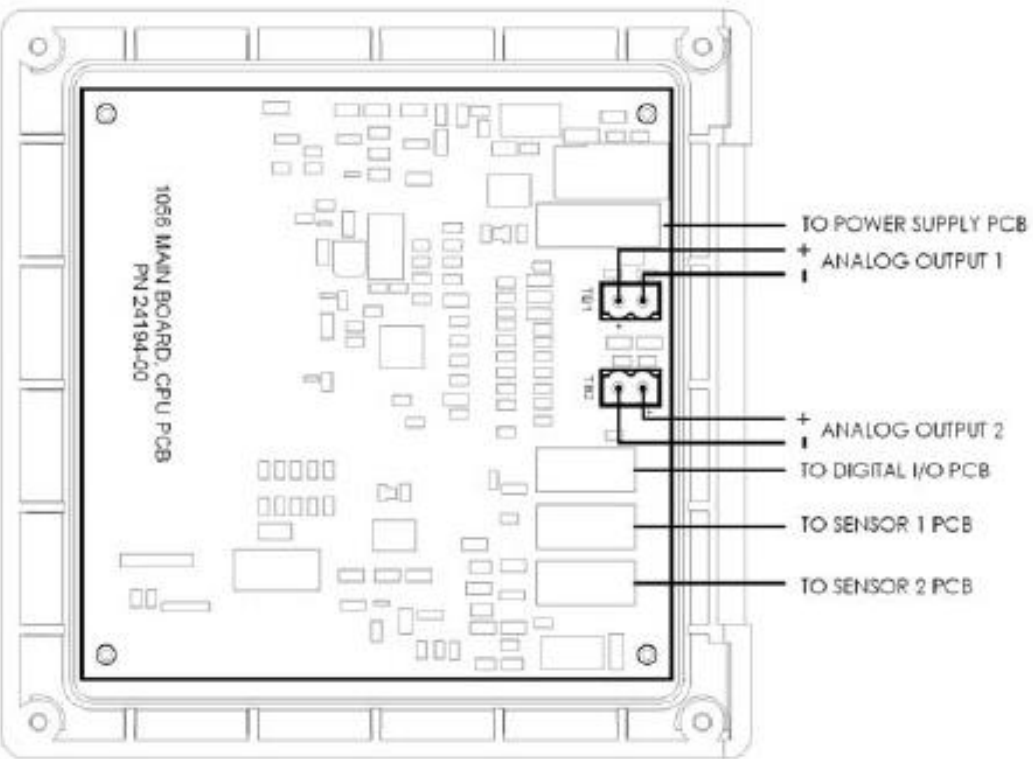

B.3. Pressure Sensor Wiring [40]

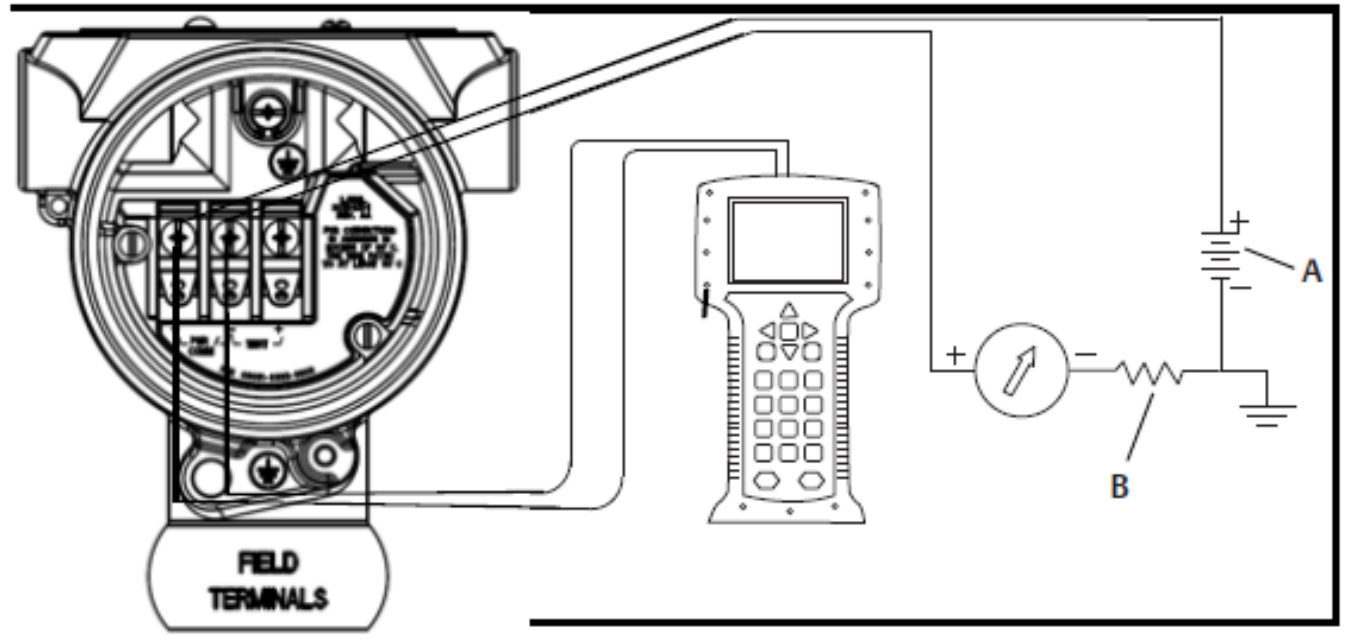

A: DC power supply.

B: $R_{L}$ equal to or greater than 250 (necessary for HART communication 


\section{B.4. DAQ Wiring}

\begin{tabular}{|c|c|c|c|c|c|c|c|c|c|c|c|c|c|c|c|c|c|}
\hline PS & $\mathrm{P}$ & ID & PS C & DIN & DB37 & S1 & S2 & S3 & S4 & S5 & S6 & S9 & S10 & S11 & S12 & S13 & S14 \\
\hline I & & SHIELD & SHIELD & 0 & & & & & & & & & & & & & \\
\hline $\mathbf{A}$ & & $24 \mathrm{~V}$ & RED & 1 & & & & & & & & & & & & & \\
\hline B & & GND & B-R/B & 2 & & & & & & & & & & & & & \\
\hline C & & $5 \mathrm{~V}$ & GREEN & 3 & & & & & & & & & & & & & \\
\hline D & & GND & G-G/B & 4 & & & & & & & & & & & & & \\
\hline $\mathbf{E}$ & & $9-15 \mathrm{~V}$ & WHITE & 5 & & & & & & & & & & & & & \\
\hline $\mathbf{F}$ & & GND & W-W/B & 6 & & & & & & & & & & & & & \\
\hline & $24+$ & & & 7 & & $\mathrm{R}$ & & & & & & & & & & & \\
\hline & $24-$ & & & 8 & & B & & & & & & & & & & & \\
\hline & & SIG & & 8 & 1 & G & & & & & & & & & & & \\
\hline & & SIG & & 20 & 20 & W & & & & & & & & & & & \\
\hline & & & & 11 & & $S$ & & & & & & & & & & & \\
\hline & & & & 12 & & $\mathrm{~N}$ & & & & & & & & & & & \\
\hline & $24+$ & & & 13 & & & $\mathrm{R}$ & & & & & & & & & & \\
\hline & $24-$ & & & 14 & & & B & & & & & & & & & & \\
\hline & & SIG & & 14 & 3 & & G & & & & & & & & & & \\
\hline & & SIG & & 12 & 22 & & $\mathrm{~W}$ & & & & & & & & & & \\
\hline & & & & 17 & & & $S$ & & & & & & & & & & \\
\hline & & & & 18 & & & $\mathrm{~N}$ & & & & & & & & & & \\
\hline & $24+$ & & & 19 & & & & $\mathrm{R}$ & & & & & & & & & \\
\hline & $24-$ & & & 20 & & & & B & & & & & & & & & \\
\hline & & SIG & & 20 & 4 & & & $\mathrm{G}$ & & & & & & & & & \\
\hline & & SIG & & 18 & 23 & & & W & & & & & & & & & \\
\hline
\end{tabular}




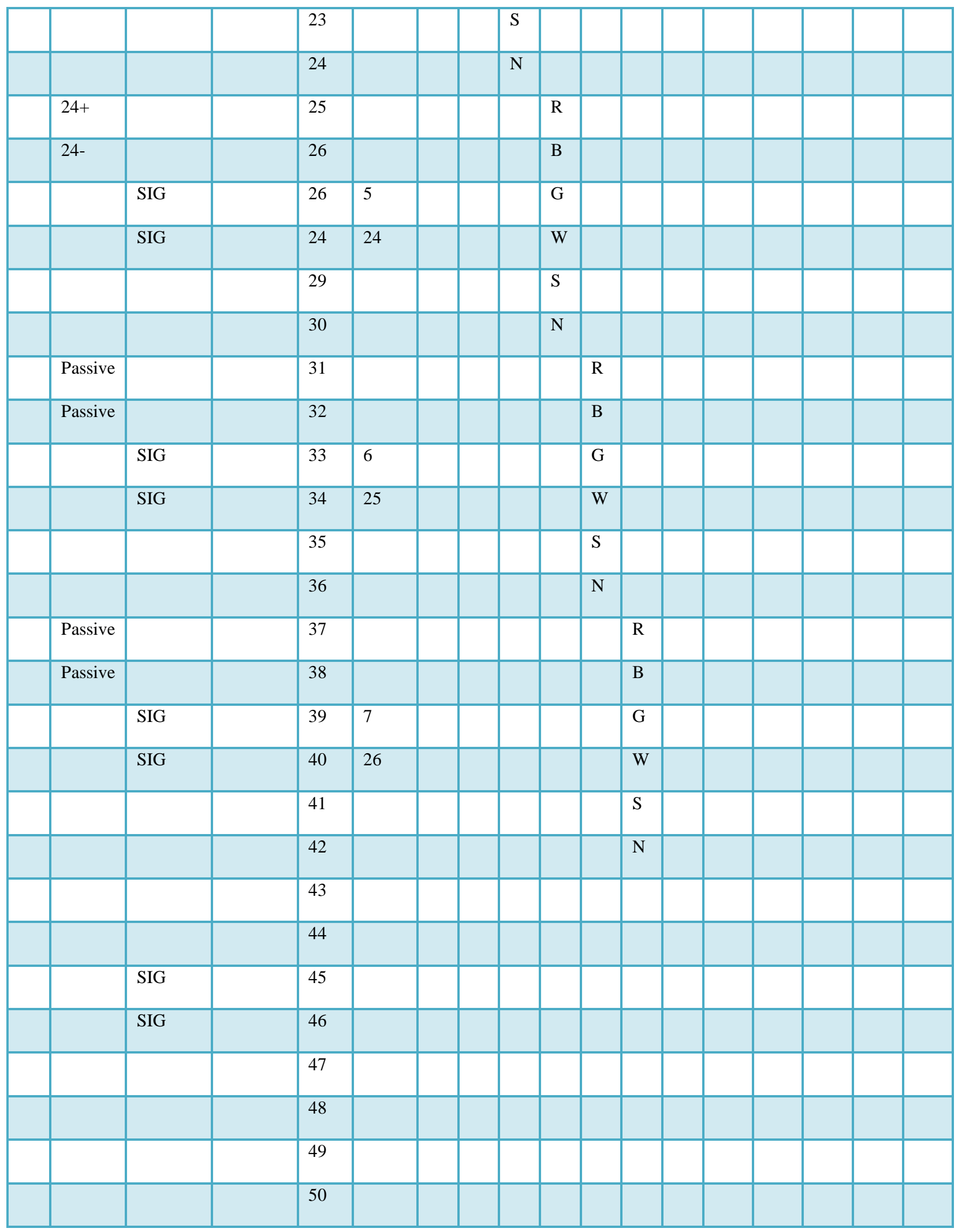




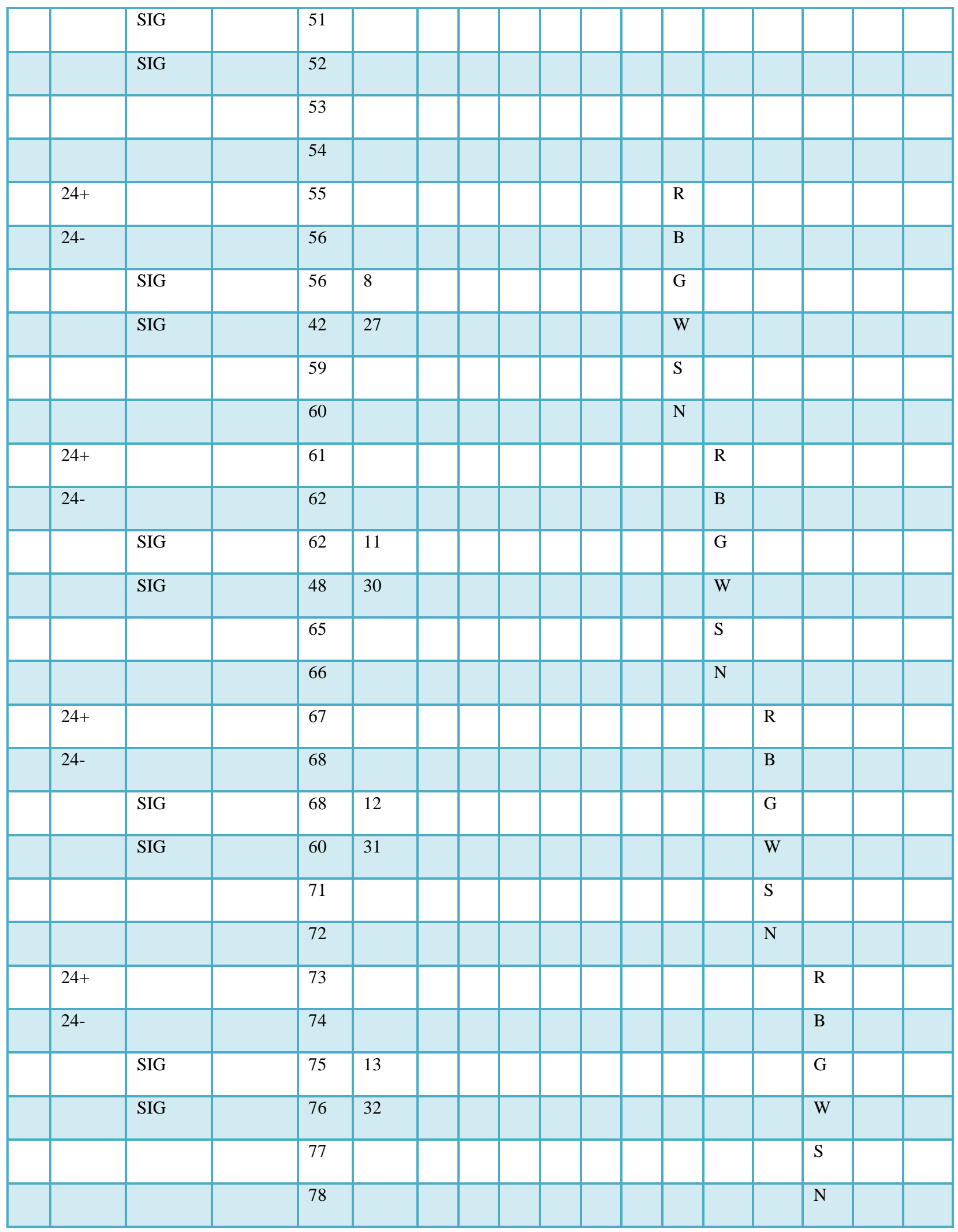




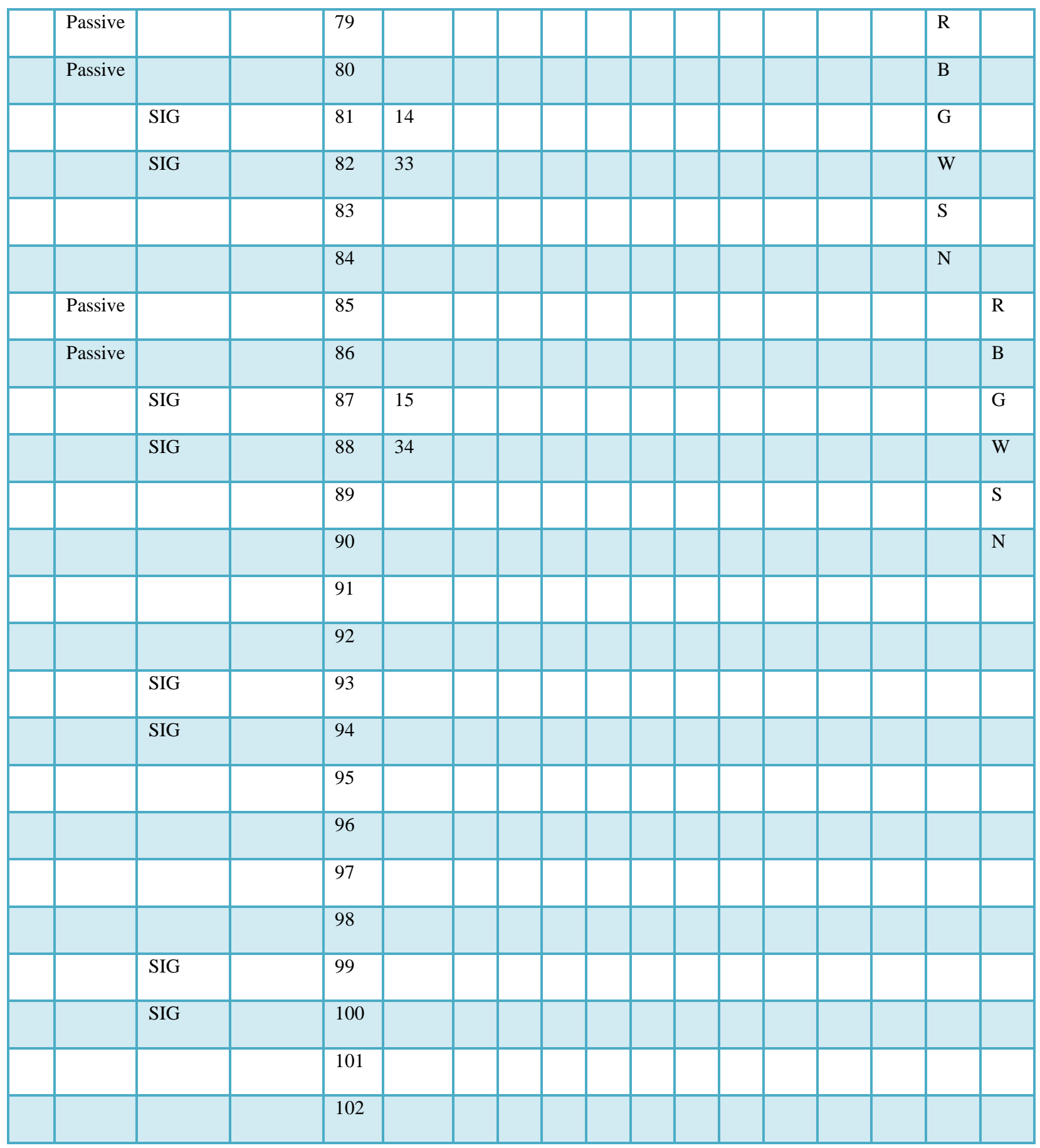

\section{SENSOR CABLE CONNECTOR IDENTIFICATION}

A=SHIELD, B=GREEN (SIG), C=WHITE (SIG), D=N/A, E=BLACK (NEG), F=RED (POS). PS = POWER SUPPLY, $\mathrm{P}=$ POWER RAILS, ID = IDENTIFICATION, DIN = DIN RAIL, DB37 = CONNECTOR, $\mathrm{S}=$ SENSOR 1 TO 16. 


\section{B.5. DAQ Wiring Diagram}

DAQ wiring is done according to the table presented in appendix B.4.

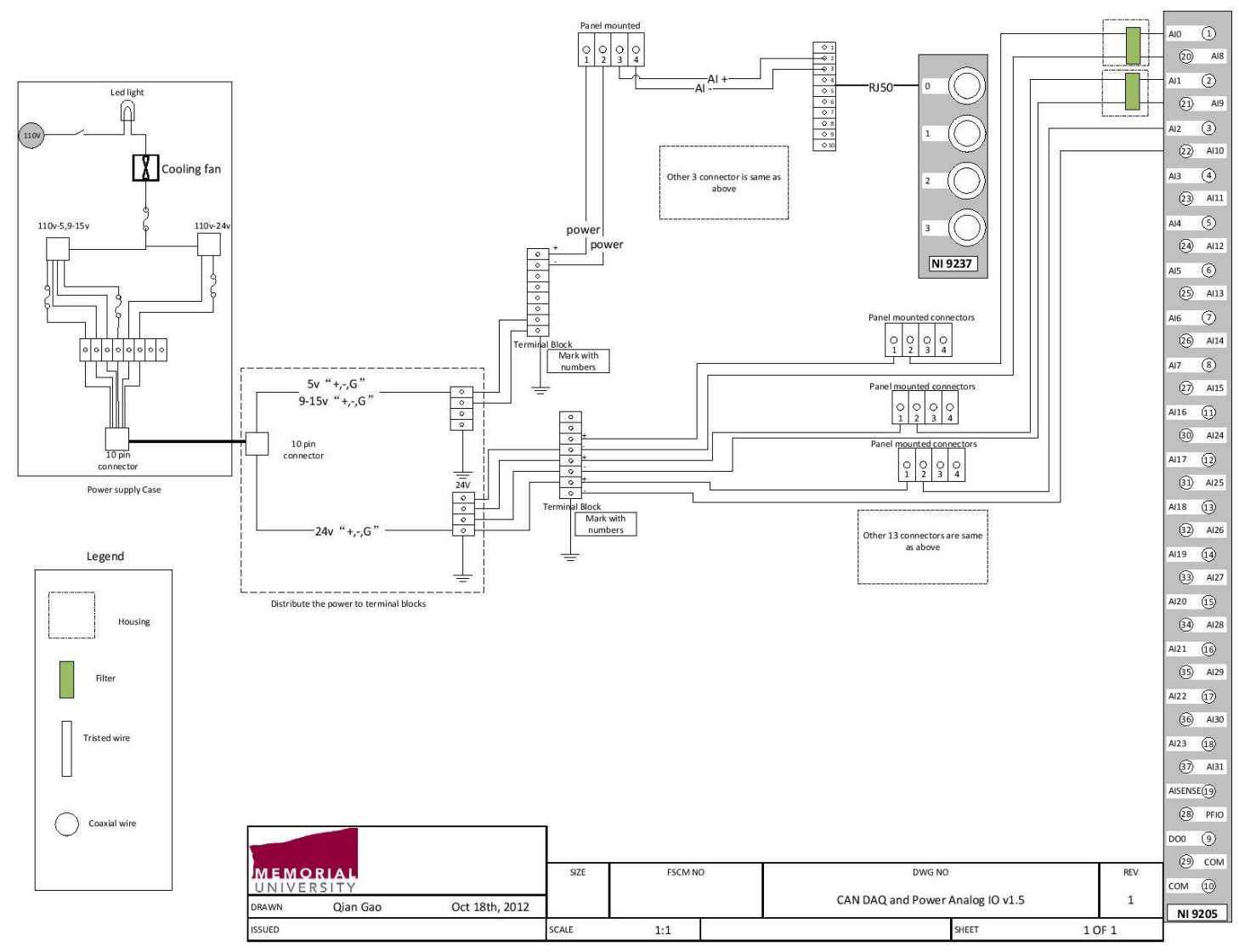

DAQ Basic Wiring [37]

\section{Appendix C DAQ Configuration}

Included in supplementary files.

\section{Appendix D Experimental Data}

Included in supplementary files. 